\title{
Evaluation of the Impact of Thin Pours on Saltstone Properties
}

\author{
A. D. Cozzi \\ C. A. Langton \\ K. M. Fox
}

September 2012

Savannah River National Laboratory Savannah River Nuclear Solutions, LLC Aiken, SC 29808

Prepared for the U.S. Department of Energy under contract number DE-AC09-08SR22470. 
SRNL-STI-2012-00522

Revision 0

\section{DISCLAIMER}

This work was prepared under an agreement with and funded by the U.S. Government. Neither the U.S. Government or its employees, nor any of its contractors, subcontractors or their employees, makes any express or implied:

1. warranty or assumes any legal liability for the accuracy, completeness, or for the use or results of such use of any information, product, or process disclosed; or

2. representation that such use or results of such use would not infringe privately owned rights; or

3. endorsement or recommendation of any specifically identified commercial product, process, or service.

Any views and opinions of authors expressed in this work do not necessarily state or reflect those of the United States Government, or its contractors, or subcontractors.

\section{Printed in the United States of America}

Prepared for

U.S. Department of Energy 
Keywords: saltstone, SDU6, hydraulic conductivity

Retention: Permanent

\title{
Evaluation of the Impact of Thin Pours on Saltstone Properties
}

\author{
A. D. Cozzi \\ C. A. Langton \\ K. M. Fox
}

September 2012

Savannah River National Laboratory Savannah River Nuclear Solutions, LLC Aiken, SC 29808

Prepared for the U.S. Department of Energy under contract number DE-AC09-08SR22470. 


\section{REVIEWS AND APPROVALS}

AUTHORS:

A. D. Cozzi, Environmental Process Development

Date

C. A. Langton, Environmental Process Development

Date

TECHNICAL REVIEW:

W. E. Daniel, Environmental Process Development

Date

APPROVAL:

K. M. Fox, Manager and Coauthor

Date

Environmental Process Development

Date

S. L. Marra, Manager

Environmental \& Chemical Process Technology Research Programs

K. H. Rosenberger, Manager

Date

SRR Closure \& Disposal Assessment

T. H. Huff, Manager

Date

SRR Engineering Projects, SDU6 
SRNL-STI-2012-00522

Revision 0

\section{EXECUTIVE SUMMARY}

The proposed Saltstone Disposal Unit 6 (SDU6) is a larger structure than the SDU4 cells and larger than the disposal units (SDU2, SDU3, and SDU5) currently in use or under construction. The additional capacity provided by SDU6 is desired to reduce life cycle costs and support site accelerated closure goals.

The larger size of the planned SDU6 could result in saltstone being placed in thinner lifts as the unit is filled. This study was performed to determine whether thinner layers of saltstone negatively impact the performance of the waste form. A larger number of cold joints could potentially result in increased drying, salt deposition, and surface oxidation. A matrix of samples was prepared to simulate thin pours ranging from 0.5 to 6 inches thick. Each sample was cured for at least 28 days prior to further characterization.

Leachability results showed that there is no obvious impact of the number of grout layers on the Leachability Index values for $\mathrm{Na}$ and $\mathrm{NO}_{3}$. The concentrations of $\mathrm{Cr}, \mathrm{NO}_{2}$, and $\mathrm{C}_{2} \mathrm{O}_{4}$ were below detection limits for all of the leachates. No attempt was made to evaluate the oxidation of these samples since no measureable $\mathrm{Cr}$ was leached, although this would appear to indicate that $\mathrm{Cr}$ in the samples remained reduced for cold joints with surfaces exposed for approximately four days.

The results of hydraulic conductivity measurements showed that the number of cold joints in the samples did not have a significant impact on the measured values for the vertical lift orientation (i.e., when the flow path is perpendicular to the cold joints). For the horizontal lift orientation (i.e., when the flow path is parallel to the cold joints), the number of cold joints in the samples also did not appear to have a significant impact on hydraulic conductivity. The measured hydraulic conductivity was faster when the flow path was parallel to the cold joints as compared to when the flow path was perpendicular to the cold joints. Percolation testing showed increased flow when the number of cold joints was increased.

Compressive strength testing showed that the maximum load at the onset of cracking was reduced by approximately $26 \%$ for those samples that contained cold joints as compared to the monolithic samples. The number of cold joints in the sample had no significant impact on the maximum load prior to cracking.

The porosity of the samples was not influenced by cold joints. This result was expected as the porosity is a material property affected by the properties of the components (premix and salt solution) and the water to premix ratio.

Overall, the only obvious impact of cold joints in the samples was to significantly increase hydraulic conductivity in the direction parallel to the cold joints. An increasing number of cold joints (thin layers) in the simulated saltstone samples did not exacerbate this effect, nor did it have a negative impact on the Leachability Indices or porosity for surfaces exposed for approximately four days. The presence of a cold joint reduced the compressive strength of the material, although this impact was seen regardless of the number of cold joints in the sample. 


\section{TABLE OF CONTENTS}

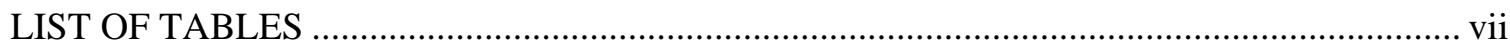

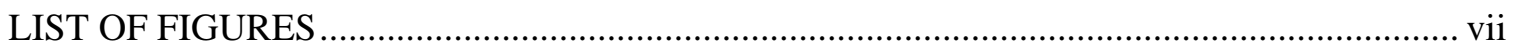

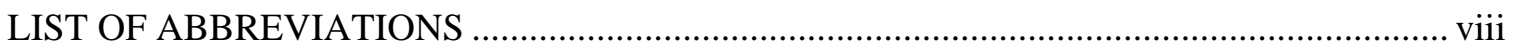

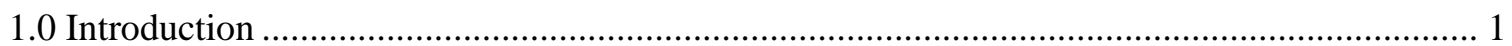

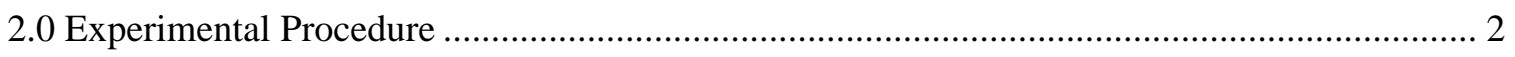

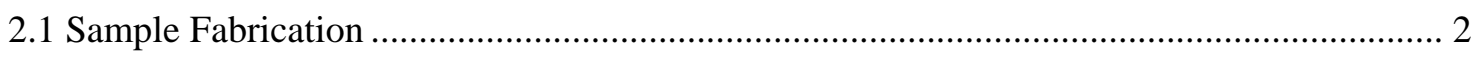

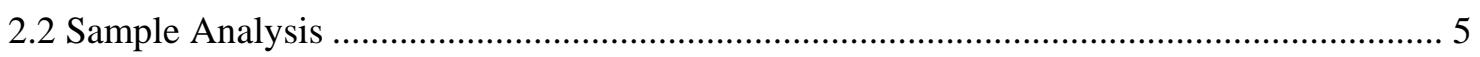

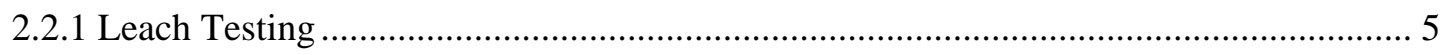

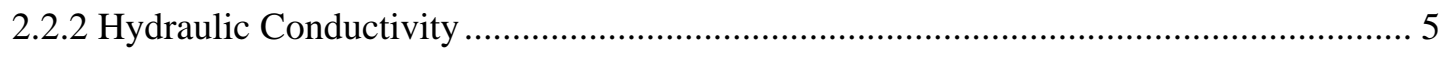

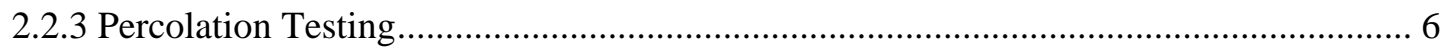

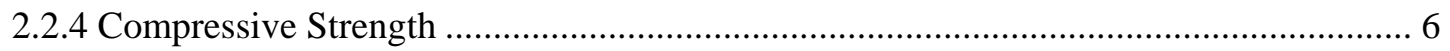

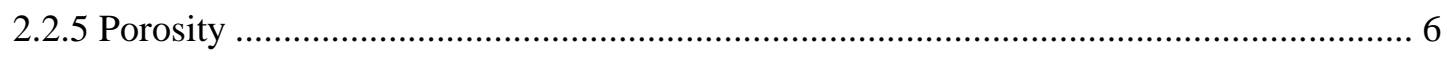

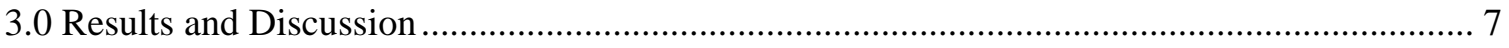

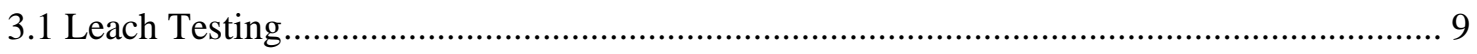

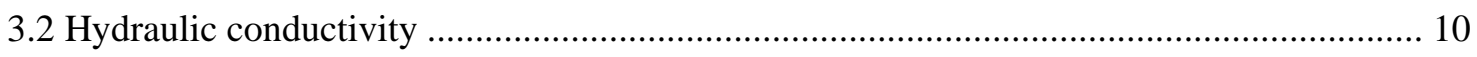

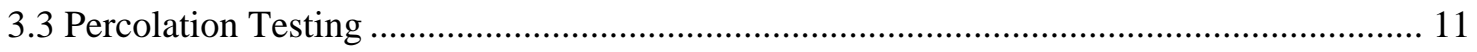

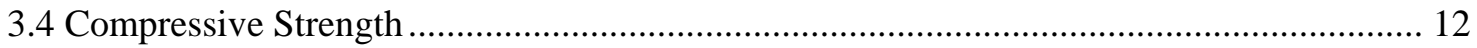

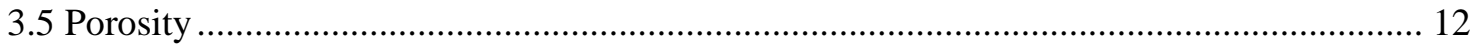

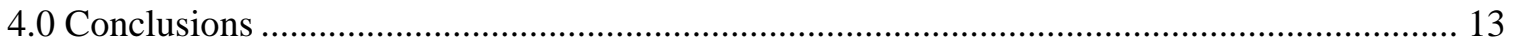

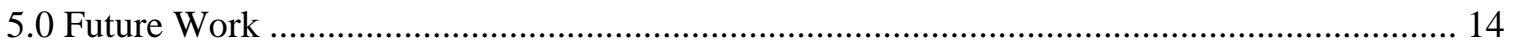

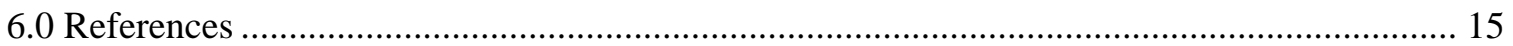




\section{LIST OF TABLES}

Table 1-1. Summary of Dimensions of Current and Proposed SDUs. ........................................... 1

Table 2-1. Nominal Saltstone Premix Blend Ratio. …................................................................. 2

Table 2-2. Simulant Salt Solution Based on CY11 WAC Analysis............................................. 2

Table 2-3. Pour Schedule for Lift into Cylinders. ........................................................................ 3

Table 2-4. Volume of Grout Needed for Each Lift for the Horizontal Samples. ........................... 4

Table 2-5. Mass of Grout Needed for Each Lift for the Horizontal Samples................................. 5

Table 2-6. Leach Testing Sample Dimensions and Leachant Volumes....................................... 5

Table 3-1. Leachability Index Values for Vertical Pour Samples.............................................. 10

Table 3-2. Summary of Hydraulic Conductivity Data for the Vertical and Horizontal Samples

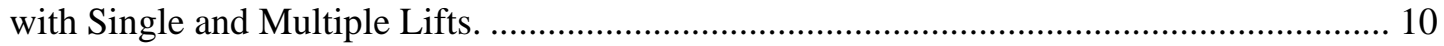

Table 3-3. Change in Water Level (mm) for Vertical Pour Percolation Test Samples. ............... 11

Table 3-4. Maximum Load at the Onset of Cracking for Vertical Pour Samples. ....................... 12

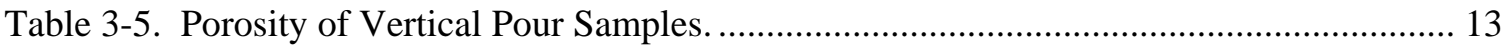

\section{LIST OF FIGURES}

Figure 2-1. Measurements needed to calculate horizontal lift volumes. ...................................... 4

Figure 2-2. Percolation test with a vertical pour sample (A) containing a 1 inch diameter hole filled with water (B) suspended (C) inside a covered vessel (D) containing additional water below the sample (E)

Figure 3-1. Single layer and multilayer vertical pour samples after demolding. Samples containing a single layer (a), 2 layers (b), 6 layers (c), and 12 layers (d) are shown............... 8

Figure 3-2. Change in Water Level (mm) for Vertical Pour Percolation Test Samples............... 11 


\section{LIST OF ABBREVIATIONS}

$\begin{array}{ll}\text { IC } & \text { Ion Chromatography } \\ \text { ICP-AES } & \text { Inductively Coupled Plasma - Atomic Emission Spectroscopy } \\ \text { PA } & \text { Performance Assessment } \\ \text { PVC } & \text { Polyvinylchloride } \\ \text { RPM } & \text { Revolutions Per Minute } \\ \text { SDU6 } & \text { Saltstone Disposal Unit 6 } \\ \text { SRNL } & \text { Savannah River National Laboratory } \\ \text { TS } & \text { Total weight percent Solids } \\ \text { WAC } & \text { Waste Acceptance Criteria }\end{array}$




\subsection{Introduction}

The proposed Saltstone Disposal Unit 6 (SDU6) is a larger structure than the SDU4 cells and larger than the disposal units (SDU2, SDU3, and SDU5) currently in use or under construction. The additional capacity provided by SDU6 is desired to reduce life cycle costs and support site accelerated closure goals.

Table 1-1 is a summary of the dimensions of the SDUs currently in use, under construction, and planned. A comparison of the parameters of the SDUs in this table shows the significant increase in scale for SDU6.

Table 1-1. Summary of Dimensions of Current and Proposed SDUs.

\begin{tabular}{|c|c|c|c|c|}
\hline Parameter & Units & $\begin{array}{c}\text { SDU4 } \\
\text { Per Cell }\end{array}$ & $\begin{array}{c}\text { SDU2/3/5 } \\
\text { Per Cell }\end{array}$ & SDU6 \\
\hline Length & $\mathrm{ft}$ & $100 *$ & -- & -- \\
\hline Width & $\mathrm{ft}$ & $100^{*}$ & $\overline{--}$ & $\overline{--}$ \\
\hline Diameter & $\overline{\mathrm{ft}}$ & -- & $150^{\dagger}$ & $375^{\mathrm{c}}$ \\
\hline Height & $\mathrm{ft}$ & $25^{*}$ & $22^{\dagger}$ & $43^{b}$ \\
\hline Slope from center & $\%$ & -- & -- & $1.5^{b}$ \\
\hline Volume & Mgal & 1.87 & $2.908^{\dagger}$ & $\sim 35.5$ \\
\hline Volume/Height & gal/in & 6,234 & 11,016 & 68,850 \\
\hline Fill rate@150 gpm grout ${ }^{\ddagger}$ & $\mathrm{in} / \mathrm{hr}$ & 1.44 & 0.82 & 0.13 \\
\hline
\end{tabular}

$\ddagger$ Assumes self-leveling

*W828992

${ }^{\dagger} \mathrm{WB} 00001 \mathrm{~K}-4$

${ }^{\mathrm{b} C}$-CC-Z-00042

${ }^{\mathrm{C} C}$-CC-Z-00039

The Task Requirements and Criteria document for conceptual design of SDU6 identified knowledge gaps associated with processing into a larger disposal unit. ${ }^{1}$ The identified knowledge gaps were coupled with a high-level risk assessment ${ }^{2}$ to document the technical assumptions, program needs, and the approach to address the needs used for the conceptual design of SDU6. ${ }^{3}$

Savannah River Remediation-Engineering Projects-SDU6 requested that the Savannah River National Laboratory (SRNL) evaluate and recommend strategies for technical issues associated with grout placement in SDU6. ${ }^{4}$ Task 3 of that request asked that SRNL perform testing to determine whether the thin grout layers that may be associated with placement in the larger diameter SDU would negatively impact the leach response and hydraulic conductivity of the saltstone because of the additional number of cold joints. A larger number of cold joints could potentially result in increased drying, salt deposition, and surface oxidation. To address this request, SRNL developed a Task Technical and Quality Assurance Plan outlining a matrix of samples to be fabricated and defining the characterization work to be performed. ${ }^{5}$

Multilayer, simulated saltstone samples were fabricated in the laboratory. Standardized test methods were used to determine the hydraulic conductivity and leaching response of these samples. Hydraulic conductivity and relative Leachability Indices were used as measures of cured saltstone quality. Compressive strength testing was also used as an indication of the overall quality of select samples after a minimum of 28 days of curing. Further details of the sample design and fabrication, characterization, and results are presented in the sections that follow. 


\subsection{Experimental Procedure}

\subsection{Sample Fabrication}

Saltstone premix is cement, granulated blast furnace slag, and fly ash blended in the ratios shown in Table 2-1. These same ratios were used for the simulated saltstone samples fabricated for this study. A large batch of premix was prepared by blending the appropriate masses of each component by shaking in a bag until visibly homogeneous. The material was then stored in a sealed bag prior to use.

Table 2-1. Nominal Saltstone Premix Blend Ratio.

\begin{tabular}{|c|c|}
\hline Premix Component & Weight Percent \\
\hline Cement & 10 \\
\hline Slag & 45 \\
\hline Fly Ash & 45 \\
\hline
\end{tabular}

A simplified salt solution was used based on the calendar year 2011 Waste Acceptance Criteria (WAC) analysis of Tank $50 \mathrm{H}^{9}$ with intentionally elevated quantities $(1000 \mathrm{mg} / \mathrm{L})$ of chromium and rhenium as a surrogate for technetium-99 (Table 2-2). The solution was prepared by adding the compounds in the order listed in the table with the exception of water. Approximately 10$20 \%$ of the water was reserved for rinsing throughout the preparation process. Chromium and rhenium were added in identical concentrations to evaluate whether a correlation exists between leaching of the two elements. Chromium was added to the salt solution as $\mathrm{Cr}^{+6}$ via sodium chromate and was reduced by the slag in the premix. This allowed for a potential evaluation of the oxidation of the samples for the various lifts. The simulated salt solution total weight percent solids (TS) is $25.13 \%$ and density is $1.207 \mathrm{~g} / \mathrm{ml}$.

Table 2-2. Simulant Salt Solution Based on CY11 WAC Analysis.

\begin{tabular}{|c|c|c|c|}
\hline Compound & $g / L$ & Component & $\mathbf{M}$ \\
\hline Water & balance & $\mathrm{Na}$ & $4.4 \mathrm{E}+00$ \\
\hline $\mathrm{KNO}_{3}$ & 0.55 & $\mathrm{Al}$ & $1.1 \mathrm{E}-01$ \\
\hline $\mathrm{NaNO}_{3}$ & 154.37 & $\mathrm{Cr}$ & 5.8E-03 \\
\hline $\mathrm{NaOH}(50 \%)$ & 142.4 & $\operatorname{Re}$ & 1.6E-03 \\
\hline $\mathrm{Al}\left(\mathrm{NO}_{3}\right)_{3} \cdot 9 \mathrm{H}_{2} \mathrm{O}$ & 42.01 & $\mathrm{~B}$ & $1.1 \mathrm{E}-02$ \\
\hline $\mathrm{NaNO}_{2}$ & 25.66 & $\mathrm{~K}$ & $5.4 \mathrm{E}-03$ \\
\hline $\mathrm{Na}_{2} \mathrm{CO}_{3}$ & 14.73 & $\mathrm{NO}_{3}$ & $2.2 \mathrm{E}+00$ \\
\hline $\mathrm{Na}_{2} \mathrm{SO}_{4}$ & 6.59 & $\mathrm{NO}_{2}$ & 3.7E-01 \\
\hline $\mathrm{Na}_{2} \mathrm{CrO}_{4}$ & 0.94 & $\mathrm{OH}$ & $1.8 \mathrm{E}+00$ \\
\hline $\mathrm{Na}_{3} \mathrm{PO}_{4} \cdot 12 \mathrm{H}_{2} \mathrm{O}$ & 1.9 & $\mathrm{CO}_{3}$ & 1.4E-01 \\
\hline $\mathrm{NaReO}_{4}$ & 0.44 & $\mathrm{SO}_{4}$ & 4.6E-02 \\
\hline $\mathrm{Na}_{2} \mathrm{C}_{2} \mathrm{O}_{4}$ & 1.24 & $\mathrm{C}_{2} \mathrm{O}_{4}$ & 9.3E-03 \\
\hline $\mathrm{H}_{3} \mathrm{BO}_{3}$ & 0.71 & $\mathrm{Cl}$ & 4.6E-03 \\
\hline $\mathrm{NaCl}$ & 0.27 & & \\
\hline
\end{tabular}

Simulated saltstone mixes using the premix in Table 2-1 and salt solution in Table 2-2 were prepared at a water to premix ratio of 0.60 by mass. 
A matrix of samples was prepared in 3 inch by 6 inch cylindrical molds. These consisted of one lift (i.e., a full mold with no cold joints) up to twelve lifts (i.e., a full mold containing eleven cold joints) with the long axis of the cylindrical molds oriented both vertically and horizontally. The appropriate amount (dependent upon the size of the lift to be poured) of premix was weighed into a bag. The appropriate amount of salt solution was weighed into a $2000 \mathrm{ml}$ beaker. A paddle mixer in a chemical fume hood was used with the blade set between 0.25 to 0.50 inches above the bottom of the $2000 \mathrm{ml}$ beaker containing the simulated salt solution. The mixer was turned on and adjusted to an initial rotational speed of $\sim 250$ revolutions per minute (RPM). A corner was cut off of the bottom of the plastic bag containing the premix and the material was slowly poured into the beaker. The rotational speed of the mixer was increased as needed to ensure wetting of the premix as it was poured into the simulated salt solution. Once all the premix material was added, the speed of the mixer was adjusted until a vortex was formed around the shaft without significantly entraining air into the mix. Mixing continued for approximately three minutes after a vortex was formed. The speed of the mixer was continually adjusted during this time to avoid significant air entrainment. After three minutes of mixing, the mixer was turned off and the material was cast in triplicate in lifts of varying heights into cylindrical molds oriented either vertically or horizontally and cured at ambient temperature.

In addition to the solid vertical lifts, triplicate samples of the vertical cylinders were cast with a Polyvinylchloride (PVC), 1 inch diameter rod in the center of the cylinder to support later percolation tests. The samples were prepared by initially pouring a 0.5 inch lift into a cylindrical mold. After three days, PVC rods were centered in each of the cylinders on top of the first lift. The subsequent lifts were poured with the corresponding solid vertical lift samples but with the grout volume reduced appropriately to account for the PVC rod.

The curing conditions of the vertical samples were managed by sealing the samples in plastic bags containing water saturated wipes to maintain a humid environment. The horizontal samples were covered in Parafilm, capped, and taped prior to curing horizontally.

Table 2-3 is the pour schedule used for each lift. The lifts were placed twice per week. For each sample, the 28 day minimum curing period began after the final lift.

Table 2-3. Pour Schedule for Lift into Cylinders.

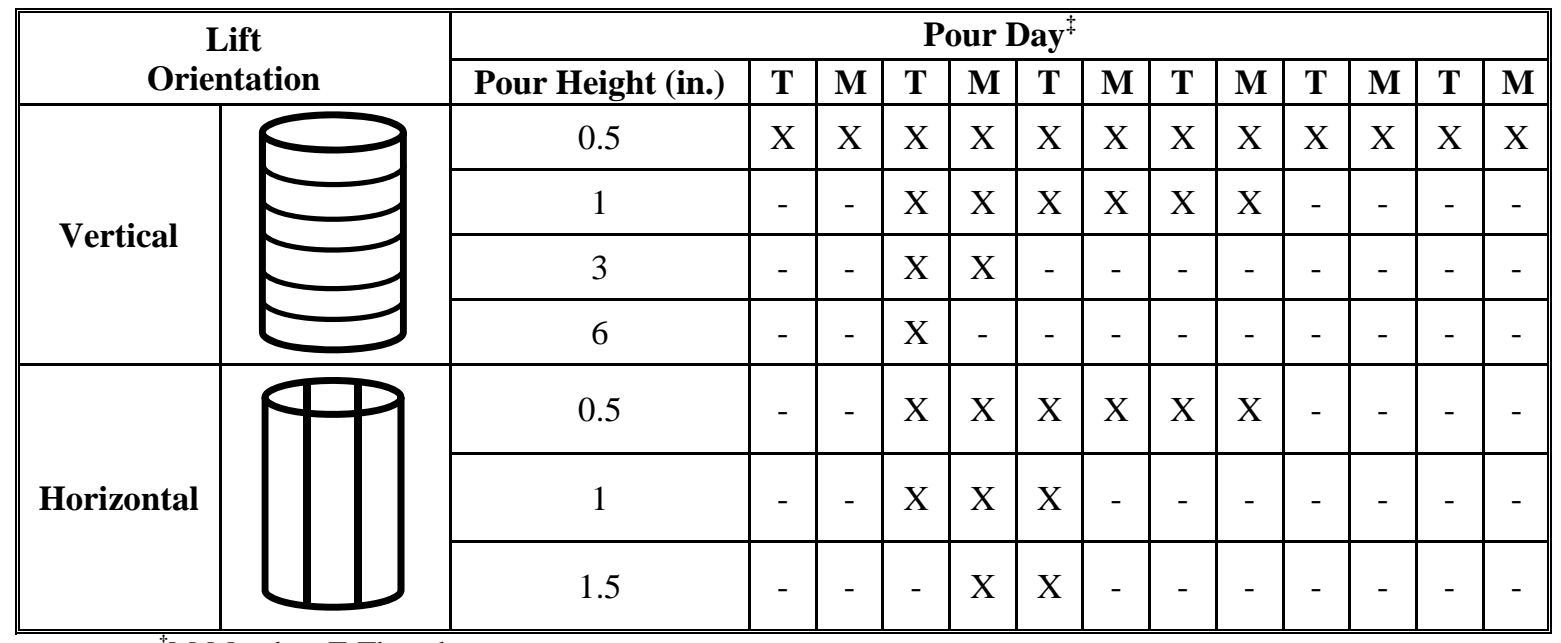

${ }^{\mp}$ M-Monday; T-Thursday 
To fill the vertical samples, the grout density and volume per lift of cylinder were used to calculate the amount of grout needed for each lift. A grout density of $1.72 \mathrm{~g} / \mathrm{cm}^{3}$ was used based on concurrent testing with a similar salt solution. ${ }^{10}$ To fill the horizontal samples, the volume per lift was calculated from the area of the chord ACD in Figure 2-1, where line BD is the cylinder diameter (3 inches) and line ED is the lift height (either 0.5 , 1, or 1.5 inches). Details of these calculations are provided in the task plan. ${ }^{5}$

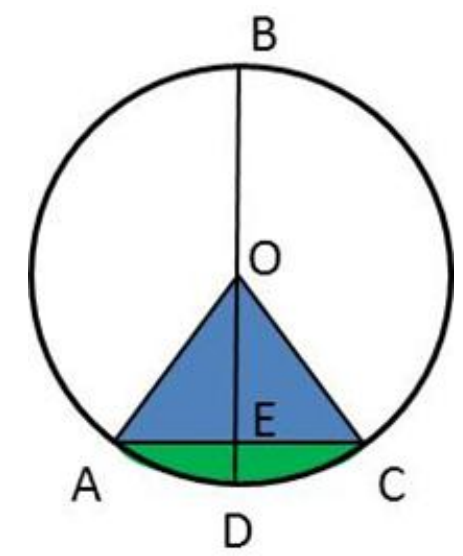

Figure 2-1. Measurements needed to calculate horizontal lift volumes.

Table 2-4 gives the volume of simulated saltstone targeted for each lift height in the horizontal cylinders. The lift intervals are $0.5,1$, and 1.5 inch, resulting in 6,3 , and 2 lifts, respectively. Table 2-5 gives the mass of grout targeted for each of the horizontal lifts. The appropriate mass of material was poured into the vertically oriented molds. The molds were then rotated to a horizontal condition to cure.

Table 2-4. Volume of Grout Needed for Each Lift for the Horizontal Samples.

\begin{tabular}{|c|c|c|c|c|c||}
\hline $\begin{array}{c}\text { Height } \\
\text { (in) }\end{array}$ & $\begin{array}{c}\text { Height } \\
\text { (cm) }\end{array}$ & $\begin{array}{c}\text { Volume } \\
\text { (ml) }\end{array}$ & $\begin{array}{c}\text { Addition } \\
\mathbf{0 . 5} \text { inch (ml) }\end{array}$ & $\begin{array}{c}\text { Addition } \\
\mathbf{1} \text { inch (ml) }\end{array}$ & $\begin{array}{c}\text { Addition } \\
\mathbf{1 . 5} \text { inch (ml) }\end{array}$ \\
\hline 0.5 & 1.27 & 76.1 & 76.1 & -- & -- \\
\hline 1.0 & 2.54 & 202.8 & 126.7 & 202.8 & -- \\
\hline 1.5 & 3.81 & 347.5 & 144.7 & -- & 347.5 \\
\hline 2.0 & 5.08 & -- & 144.7 & 289.4 & -- \\
\hline 2.5 & 6.35 & -- & 126.7 & -- & -- \\
\hline 3.0 & 7.62 & -- & 76.1 & 202.8 & 347.5 \\
\hline
\end{tabular}


Table 2-5. Mass of Grout Needed for Each Lift for the Horizontal Samples.

\begin{tabular}{|c|c|c|c|c|c||}
\hline $\begin{array}{c}\text { Height } \\
\text { (in) }\end{array}$ & $\begin{array}{c}\text { Height } \\
\text { (cm) }\end{array}$ & $\begin{array}{c}\text { Volume } \\
\text { (ml) }\end{array}$ & $\begin{array}{c}\text { Additional } \\
\mathbf{0 . 5} \text { inch (g) }\end{array}$ & $\begin{array}{c}\text { Additional } \\
\mathbf{1} \text { inch (g) }\end{array}$ & $\begin{array}{c}\text { Additional } \\
\mathbf{1 . 5} \text { inch (g) }\end{array}$ \\
\hline 0.5 & 1.27 & 76.1 & 131.2 & -- & -- \\
\hline 1.0 & 2.54 & 202.8 & 218.2 & 349.4 & -- \\
\hline 1.5 & 3.81 & 347.5 & 249.3 & -- & 498.7 \\
\hline 2.0 & 5.08 & -- & 249.3 & 598.7 & -- \\
\hline 2.5 & 6.35 & -- & 218.2 & -- & -- \\
\hline 3.0 & 7.62 & -- & 131.2 & 349.4 & 498.7 \\
\hline
\end{tabular}

\subsection{Sample Analysis}

\subsubsection{Leach Testing}

Degradation and contaminant movement though saltstone is used in the Performance Assessment (PA) to model the release of contaminants to the environment. ${ }^{11}$ Leachability measurements were performed following the ANSI/ANS 16-1 standard. ${ }^{12}$ Leach testing specimens (the vertical pour samples only) were prepared in triplicate at room temperature and cured at room temperature for at least 28 days. The samples were right cylinders, with dimensions as given in Table 2-6. The volume of leachant used during each interval is also included in Table 2-6.

Table 2-6. Leach Testing Sample Dimensions and Leachant Volumes.

\begin{tabular}{|c|c|c|c|c|c|}
\hline $\begin{array}{c}\text { Vertical } \\
\text { Pour Height } \\
\text { (in.) }\end{array}$ & Sample ID & $\begin{array}{c}\text { Diameter } \\
(\mathbf{m m})\end{array}$ & $\begin{array}{c}\text { Height } \\
\mathbf{( m m})\end{array}$ & $\begin{array}{c}\text { Mass } \\
\mathbf{( g )}\end{array}$ & $\begin{array}{c}\text { Leachant Volume } \\
\mathbf{( m l )}\end{array}$ \\
\hline \multirow{3}{*}{0.5} & $0.5-5$ & 76.33 & 119.51 & 943.8 & 3781.0 \\
\cline { 2 - 6 } & $0.5-6$ & 76.78 & 122.12 & 968.1 & 3871.7 \\
\cline { 2 - 6 } & $0.5-13$ & 77.27 & 120.31 & 960.8 & 3858.4 \\
\hline \multirow{3}{*}{1.0} & $1.0-4$ & 76.03 & 127.91 & 989.9 & 4018.1 \\
\cline { 2 - 6 } & $1.0-5$ & 76.54 & 128.63 & 1012.9 & 4006.5 \\
\cline { 2 - 6 } & $1.0-6$ & 77.87 & 129.79 & 1010.8 & 3981.0 \\
\hline \multirow{3}{*}{3.0} & $3.0-4$ & 77.26 & 129.53 & 1020.2 & 3953.2 \\
\cline { 2 - 6 } & $3.0-5$ & 76.16 & 128.27 & 1009.0 & 4013.9 \\
\cline { 2 - 6 } & $3.0-6$ & 76.19 & 127.69 & 995.7 & 4003.7 \\
\hline \multirow{3}{*}{6.0} & $6.0-4$ & 76.88 & 127.31 & 1002.5 & 4040.9 \\
\cline { 2 - 6 } & $6.0-5$ & 76.44 & 129.55 & 1005.7 & 4000.8 \\
\cline { 2 - 6 } & $6.0-6$ & 77.30 & 128.83 & 1013.8 & 4019.4 \\
\hline
\end{tabular}

The leachates were at ambient temperature at the end of each interval. Aliquots of approximately $125 \mathrm{ml}$ of each leachate were analyzed by Inductively Coupled Plasma - Atomic Emission Spectroscopy (ICP-AES) for $\mathrm{Cr}, \mathrm{Na}$, and Re concentrations, and by Ion Chromatography (IC) for $\mathrm{NO}_{2}, \mathrm{NO}_{3}$, and $\mathrm{C}_{2} \mathrm{O}_{4}$ concentrations. Each aliquot was measured twice by ICP-AES with the mean of the two measurements used in calculating the Leachability Index. Single measurements were performed by IC. Blank samples consisting only of deionized water were also included for analysis with the leachate aliquots for each leaching interval.

\subsubsection{Hydraulic Conductivity}

The transport of water through saltstone is an input parameter to the numerical model that supports the PA. ${ }^{13}$ Samples from each of the horizontal and vertical test conditions were 
measured to determine the effect of the saltstone placement on the hydraulic conductivity following ASTM D-5084. ${ }^{14}$ Due to the number of samples to be tested in triplicate, the procedure was carried out by AMEC Environment \& Infrastructure (Atlanta, Georgia).

\subsubsection{Percolation Testing}

Vertical pour samples that were cast with a nonreactive, 1 inch diameter rod to displace a portion of the simulated saltstone were tested in triplicate using a percolation-type test where the void space of the demolded sample was filled with water and allowed to drain. A modified version of the field test was used to measure the unpressurized flow through each sample. ${ }^{15}$ The PVC rods were removed and the samples were demolded and suspended in a covered vessel with water below the bottom of the sample (Figure 2-2). The hole left after removal of the rod was filled with water and the water level within the hole was then monitored over several days. The water was not replenished during the test.

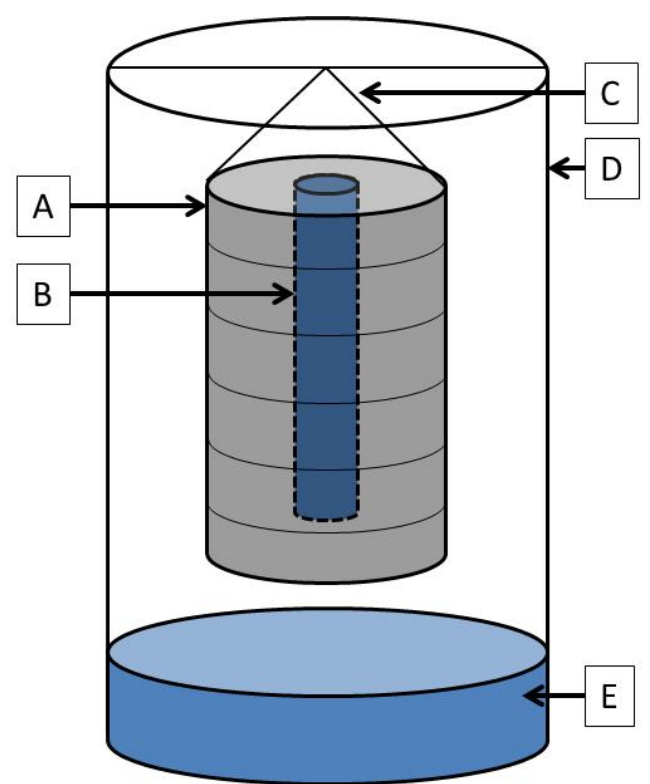

Figure 2-2. Percolation test with a vertical pour sample (A) containing a 1 inch diameter hole filled with water (B) suspended (C) inside a covered vessel (D) containing additional water below the sample (E).

\subsubsection{Compressive Strength}

Compressive strength is not a required property for saltstone permitting. However, compressive strength is commonly used as an indication of the overall quality (mix design and preparation) of the sample. After curing for a minimum of 28 days, vertical pour samples were removed from the molds and tested for compressive strength in triplicate following a procedure based on ASTM C39/39M. ${ }^{16}$ The maximum load prior to cracking was recorded and used as a relative method of comparison among the samples with varying numbers of cold joints.

\subsubsection{Porosity}

Porosity is a material property that is used as an input parameter to the PA. ${ }^{11}$ After curing for a minimum of 28 days, vertical pour samples were removed from their molds and the porosity was measured following the method developed in prior work. ${ }^{17}$ The entire cylinder was measured 
rather than a subsample in order to capture the effect of the thin layers. In addition, the density calculation was confirmed by determining the volume of the cylinder by geometrical methods.

\subsection{Results and Discussion}

Example photographs of the multilayer samples after demolding are shown in Figure 3-1. The cold joints between the layers were visible after demolding. 


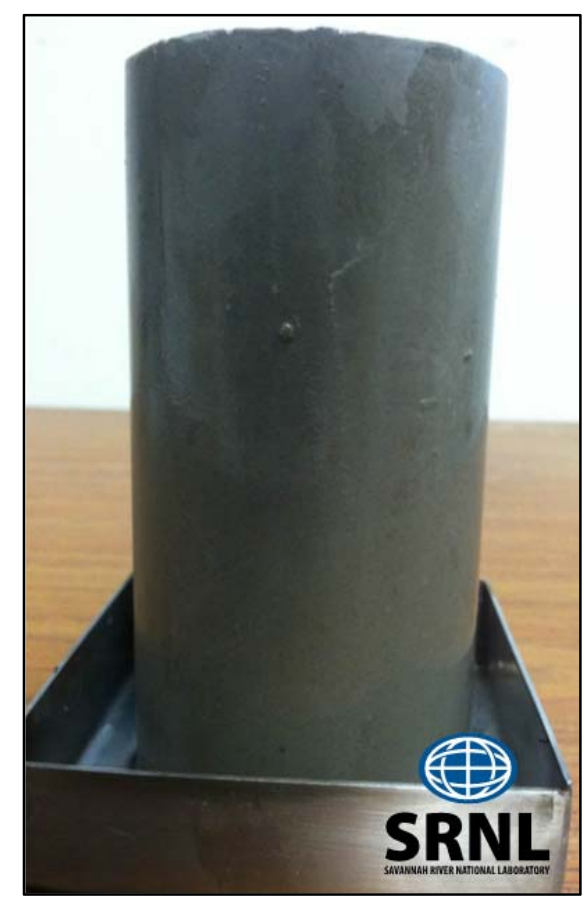

(a)

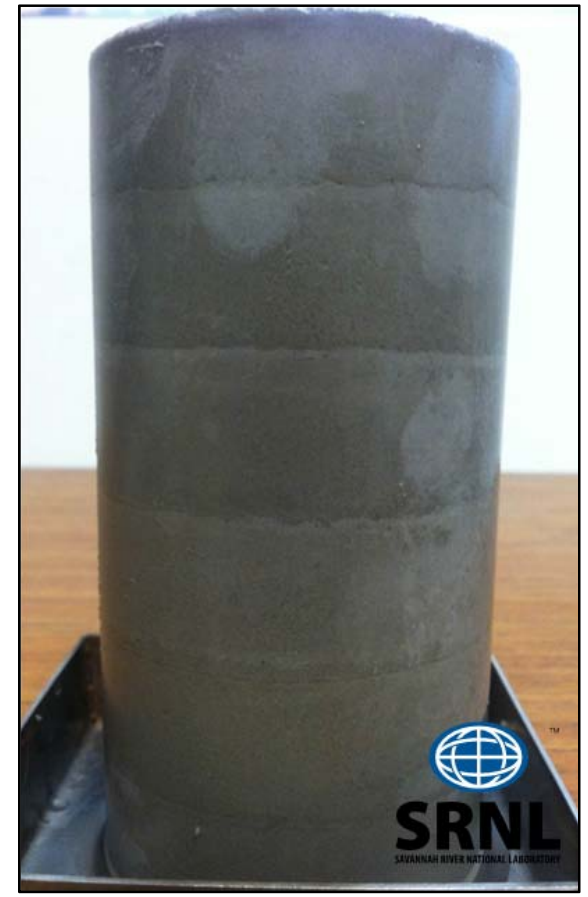

(c)

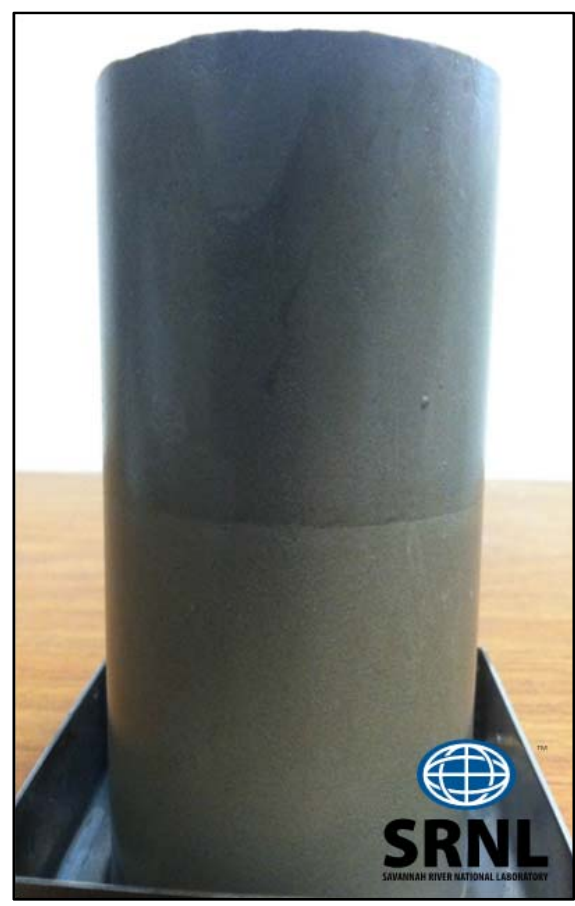

(b)

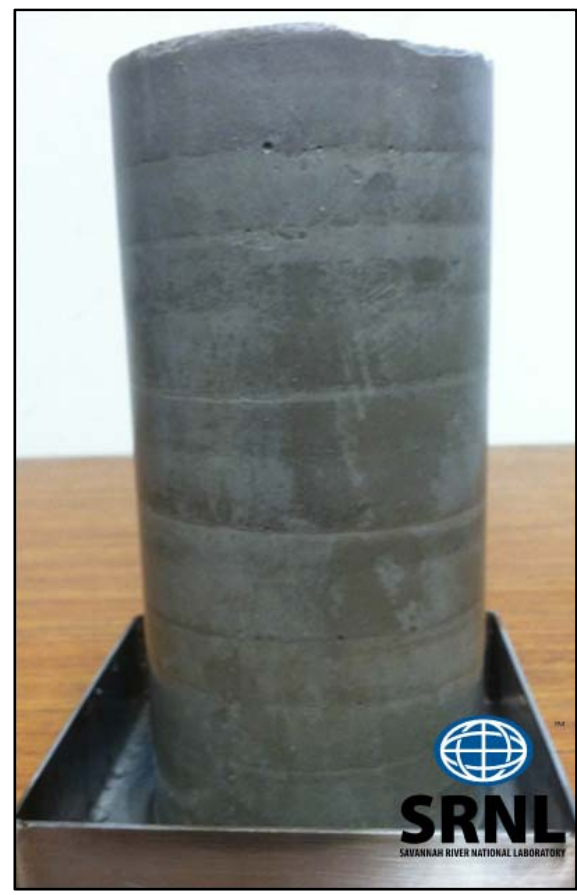

(d)

Figure 3-1. Single layer and multilayer vertical pour samples after demolding. Samples containing a single layer (a), 2 layers (b), 6 layers (c), and 12 layers (d) are shown. 


\subsection{Leach Testing}

Per ANSI/ANS 16-1, ${ }^{12}$ leaching intervals of 2, 7, 24, 48, 72, 96, and 120 hours were used. The last leaching interval for the 1 inch and 3 inch pours had to be extended from 120 hours to 168 hours. Although this deviates from the procedure, Leachability Indices were calculated for these samples by simply using the extended seventh interval. The beginning and end dates and times for each leaching interval were recorded in a controlled laboratory notebook. ${ }^{a}$ The surfaces of the specimens appeared smooth both before and after leaching. No obvious changes in the shape or dimensions of the specimens were observed. No undissolved solids were visible in the leachates. The measured concentrations of each contaminant present in the leachate aliquots after each interval for each sample are given in Appendix A.

The concentrations of $\mathrm{Cr}, \mathrm{Na}, \mathrm{Re}, \mathrm{NO}_{2}$, and $\mathrm{C}_{2} \mathrm{O}_{4}$ were below detection limits for all of the blanks that were analyzed with the leachates. The concentrations of $\mathrm{NO}_{3}$ were below detection limits for all of the blanks except for those submitted with the 6 inch pour samples leached for 30 seconds, 7 hours, and 120 hours. These values were relatively low $(<13 \mathrm{mg} / \mathrm{L})$ and were considered to have no impact on the results of the study.

The concentrations of $\mathrm{Cr}, \mathrm{NO}_{2}$, and $\mathrm{C}_{2} \mathrm{O}_{4}$ were below detection limits for all of the leachates. Therefore, Leachability Index values were not calculated for these contaminates. The Re concentration was below the detection limit for samples 0.5-6 and 0.5-13 after the initial 30 seconds of leaching, and was below the detection limit for sample 0.5-13 after the 120 hour leaching interval. A Leachability Index value is therefore not reported for sample 0.5-13.

Since the $\mathrm{Cr}$ concentrations were all below the detection limit, it is difficult to draw any correlation between leaching of $\mathrm{Cr}$ and Re. A concurrent study has shown that Re does not leach congruently with $\mathrm{Cr}$ and is not a good surrogate for Tc. ${ }^{18}$ No attempt was made at evaluating the oxidation of these samples since no measureable $\mathrm{Cr}$ was leached, although this would appear to indicate that $\mathrm{Cr}$ in the samples remained reduced.

The Leachability Index for each specimen was calculated following ANSI/ANS 16-1. ${ }^{12}$ The resulting values are given in Table 3-1. Note again that the final interval for samples of the 1 inch and 3 inch pours was 168 hours rather than 120 hours. The results show that there is no obvious impact of the number of grout layers on the Leachability Index values.

${ }^{\text {a }}$ SRNL-NB-2012-00059 
Table 3-1. Leachability Index Values for Vertical Pour Samples.

\begin{tabular}{||c|c|c|c|c||}
\hline $\begin{array}{c}\text { Vertical Pour } \\
\text { Height } \\
\text { (in.) }\end{array}$ & Sample ID & \multicolumn{3}{|c|}{ Leachability Index } \\
\cline { 3 - 5 } & & $\mathbf{N a}$ & $\mathbf{R e}$ & $\mathbf{N O}_{3}$ \\
\hline \multirow{3}{*}{0.5} & $0.5-5$ & 9.0 & 10.0 & 9.1 \\
\cline { 2 - 5 } & $0.5-6$ & 8.8 & 9.8 & 8.8 \\
\cline { 2 - 5 } & $0.5-13$ & 9.0 & $10.0^{*}$ & 9.1 \\
\hline \multirow{3}{*}{1.0} & $1.0-4$ & 8.7 & 9.9 & 8.9 \\
\cline { 2 - 5 } & $1.0-5$ & 8.9 & 10.1 & 9.0 \\
\cline { 2 - 5 } & $1.0-6$ & 8.9 & 10.1 & 9.1 \\
\hline \multirow{3}{*}{6.0} & $3.0-4$ & 8.9 & 10.1 & 9.2 \\
\cline { 2 - 5 } & $3.0-5$ & 8.8 & 10.0 & 9.1 \\
\cline { 2 - 5 } & $3.0-6$ & 8.7 & 9.9 & 8.9 \\
\hline \multirow{3}{*}{6.0} & $6.0-4$ & 8.8 & 9.9 & 8.9 \\
\cline { 2 - 5 } & $6.0-5$ & 8.6 & 9.7 & 8.6 \\
\cline { 2 - 5 } & $6.0-6$ & 9.0 & 10.0 & 9.2 \\
\hline
\end{tabular}

*Uses time intervals 1-6 since the Re concentration was below the detection limit for interval 7.

\subsection{Hydraulic conductivity}

Hydraulic conductivity measurements following ASTM D5084 were completed by AMEC. A copy of the test report from AMEC is included as Appendix B. A summary of the hydraulic conductivity data is presented in Table 3-2.

Table 3-2. Summary of Hydraulic Conductivity Data for the Vertical and Horizontal Samples with Single and Multiple Lifts.

\begin{tabular}{|c|c|c|c|c|c|}
\hline \multirow{2}{*}{$\begin{array}{c}\text { Lift } \\
\text { Orientation }\end{array}$} & \multirow{2}{*}{$\begin{array}{l}\text { Pour Height } \\
\text { (in.) }\end{array}$} & \multicolumn{4}{|c|}{ Hydraulic Conductivity $(\mathrm{cm} / \mathrm{sec})$ at $20^{\circ} \mathrm{C}$} \\
\hline & & Replicate 1 & Replicate 2 & Replicate 3 & Mean \\
\hline \multirow{4}{*}{ Vertical } & 0.5 & $1.6 \mathrm{E}-9$ & 4.2E-9 & $3.1 \mathrm{E}-9$ & 3.0E-9 \\
\hline & 1 & 2.5E-9 & 2.2E-9 & $4.0 \mathrm{E}-9$ & 2.9E-9 \\
\hline & 3 & 2.3E-9 & 2.0E-9 & $1.4 \mathrm{E}-9$ & $1.9 \mathrm{E}-9$ \\
\hline & 6 & $5.9 \mathrm{E}-11$ & $1.6 \mathrm{E}-9$ & $2.6 \mathrm{E}-10$ & 6.4E-10 \\
\hline \multirow{3}{*}{ Horizontal } & 0.5 & $3.6 \mathrm{E}-8$ & 4.7E-8 & 3.7E-8 & $4.0 \mathrm{E}-8$ \\
\hline & 1 & $4.0 \mathrm{E}-8$ & $2.4 \mathrm{E}-7$ & $1.8 \mathrm{E}-7$ & $1.5 \mathrm{E}-7$ \\
\hline & 1.5 & $4.0 \mathrm{E}-8$ & $2.0 \mathrm{E}-7$ & $1.4 \mathrm{E}-8$ & 8.5E-8 \\
\hline
\end{tabular}

The number of cold joints in the samples does not appear to have a significant impact on hydraulic conductivity for the vertical lift orientation (i.e., when the flow path is perpendicular to the cold joints). Note that there is more variability in the measurements for the 6 inch vertical pour samples (i.e., the monolithic pours). This is likely due to these values being so low that they are near the limit of what can be measured using this technique. For the horizontal lift orientation (i.e., when the flow path is parallel to the cold joints), the number of cold joints in the samples again does not appear to have a significant impact on hydraulic conductivity. Hydraulic conductivity appears to be somewhat faster when the flow path is parallel to the cold joints as compared to when the flow path is perpendicular to the cold joints. 
SRNL-STI-2012-00522

Revision 0

\subsection{Percolation Testing}

The results of the percolation testing for the vertical pour samples are given in Table 3-3.

Table 3-3. Change in Water Level (mm) for Vertical Pour Percolation Test Samples.

\begin{tabular}{||c|c|c|c|c|c|c|c|c|c||}
\hline \multirow{2}{*}{$\begin{array}{c}\text { Lift } \\
\text { Orientation }\end{array}$} & Pour & \multicolumn{10}{|c|}{ Hours } \\
\cline { 2 - 11 } & Height (in.) & $\mathbf{1}$ & $\mathbf{8}$ & $\mathbf{2 4}$ & $\mathbf{3 2}$ & $\mathbf{4 8}$ & $\mathbf{5 6}$ & $\mathbf{7 2}$ & $\mathbf{8 0}$ \\
\hline \multirow{3}{*}{ Vertical } & 0.5 & 140 & 133 & 108 & 102 & 76 & 70 & 44 & 38 \\
\cline { 2 - 11 } & 1 & 140 & 137 & 127 & 121 & 119 & 116 & 113 & 110 \\
\cline { 2 - 10 } & 3 & 140 & 138 & 135 & 133 & 132 & 130 & 129 & 125 \\
\cline { 2 - 11 } & 6 & 140 & 138 & 135 & 133 & 130 & 129 & 125 & 124 \\
\hline
\end{tabular}

The sample with 0.5 inch lifts lost more water than the other samples ( 102 mm). The sample with 1 inch lifts lost $\sim 30 \mathrm{~mm}$, and the samples with 3 and 6 inch lifts lost $\sim 15 \mathrm{~mm}$. These results are presented graphically in Figure 3-2.

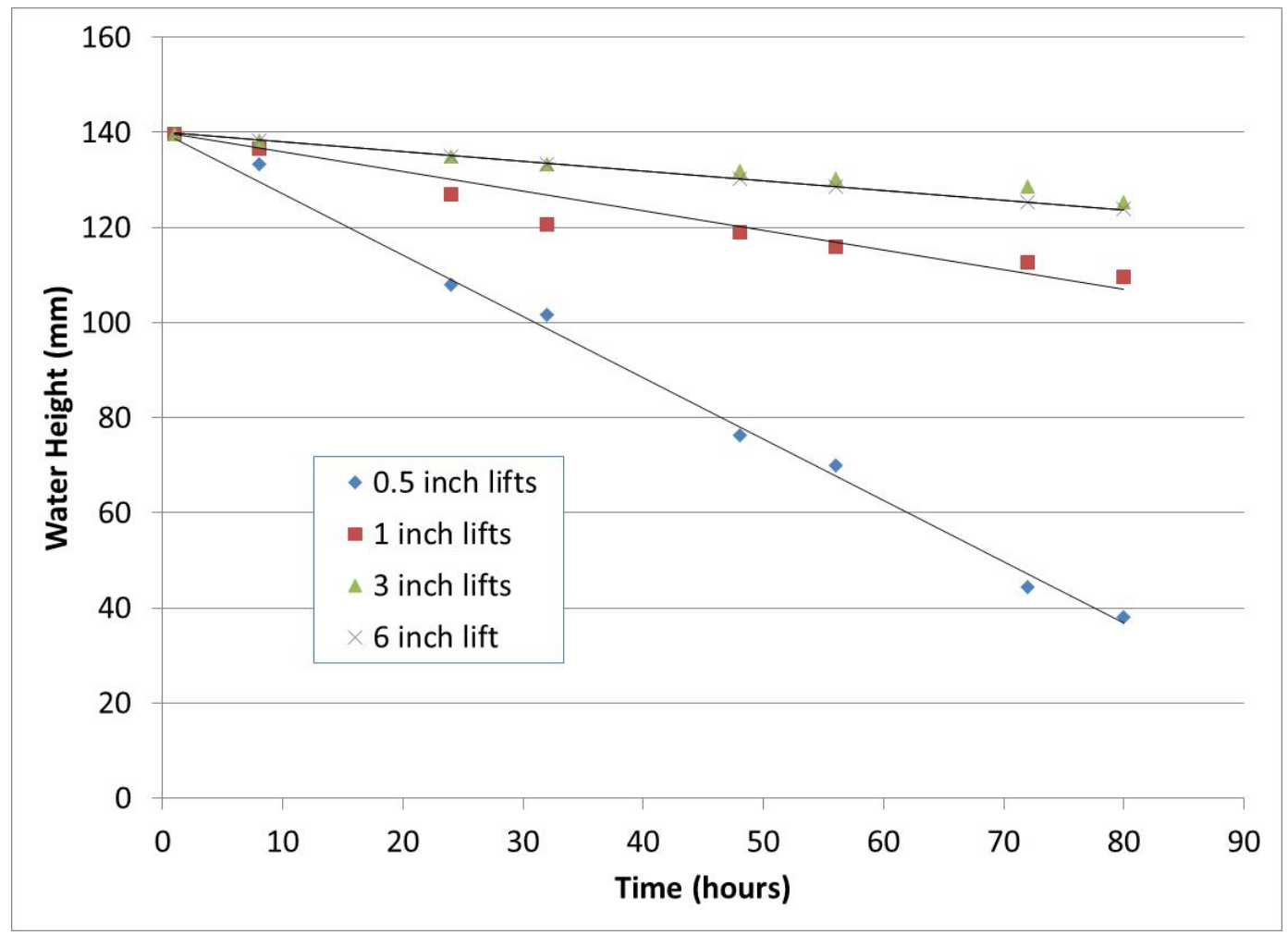

Figure 3-2. Change in Water Level (mm) for Vertical Pour Percolation Test Samples.

As opposed to the hydraulic conductivity tests on samples with vertical lifts where the sides of the samples are constrained to force flow perpendicular to the cold joints (see Section 3.2), the percolation method permitted flow parallel to or through the cold joints, more akin to the hydraulic conductivity testing of the samples with horizontally placed lifts. While the number of lifts had no obvious impact on the hydraulic conductivity results, the percolation results do show increased flow when the number of cold joints is increased. 


\subsection{Compressive Strength}

Vertical pour samples were removed from the molds after curing for a minimum of 28 days and tested for compressive strength in triplicate following a procedure based on ASTM C39/39M. ${ }^{16}$ The maximum load prior to cracking was recorded for use as a relative method of comparison among the samples with varying numbers of cold joints. The resulting data are given in Table 3-4.

Table 3-4. Maximum Load at the Onset of Cracking for Vertical Pour Samples.

\begin{tabular}{|c|c|c|c|c|}
\hline $\begin{array}{c}\begin{array}{c}\text { Pour Height } \\
\text { (in.) }\end{array} \\
\end{array}$ & Sample ID & $\begin{array}{c}\text { Max. Load } \\
\text { (lb) }\end{array}$ & $\begin{array}{c}\text { Max. Load } \\
\text { (kN) }\end{array}$ & $\begin{array}{c}\text { Mean Max. Load } \\
(k N)\end{array}$ \\
\hline \multirow{3}{*}{0.5} & $0.5-7$ & 9820 & 43.7 & \multirow{3}{*}{43.9} \\
\hline & $0.5-8$ & 9869 & 43.9 & \\
\hline & $0.5-9$ & 9920 & 44.1 & \\
\hline \multirow{3}{*}{1} & $1.0-7$ & 9643 & 42.9 & \multirow{3}{*}{42.5} \\
\hline & $1.0-8$ & 9512 & 42.3 & \\
\hline & $1.0-9$ & 9497 & 42.2 & \\
\hline \multirow{3}{*}{3} & $3.0-7$ & 9618 & 42.8 & \multirow{3}{*}{42.1} \\
\hline & $3.0-8$ & 9449 & 42.0 & \\
\hline & $3.0-9$ & 9333 & 41.5 & \\
\hline \multirow{3}{*}{6} & $6.0-7$ & 11,697 & 52.0 & \multirow{3}{*}{54.0} \\
\hline & $6.0-8$ & 12,838 & 57.1 & \\
\hline & $6.0-9$ & 11,888 & 52.9 & \\
\hline
\end{tabular}

A review of the data in Table 3-4 shows that the maximum load at the onset of cracking was reduced for those samples that contained cold joints (i.e., those with $0.5,1$, and 3 inch pour heights). The monolithic sample (i.e., the 6 inch pour height) had a mean maximum load prior to cracking that was about $26 \%$ greater than the samples with cold joints. The number of cold joints in the sample (from 1 in the samples with a 3 inch pour height to 11 in the samples with a 0.5 inch pour height) appears to have no significant impact on the maximum load prior to cracking.

\subsection{Porosity}

Vertical pour samples were removed from the molds after curing for a minimum of 28 days. The porosity was measured using the method developed in prior saltstone support. ${ }^{17}$ The sample was demolded, weighed, and dried to a constant mass. The mass loss, assumed to be pore water, was used to calculate the mass of the pore solution, assumed to be the salt solution in Section 2.1. The density of the salt solution was used to calculate the volume of the pore solution. The volume of the sample was determined from the geometric measurements of the cylinders. The sample porosity given in Table 3-5 was calculated by dividing the volume of pore solution by the sample volume. A review of Table 3-5 shows that the porosity of the samples was not influenced by the number of cold joints. This result was expected as the porosity is a material property affected by the properties of the components (premix and salt solution) and the water to premix ratio, and because the cold joints account for a very small portion of the total sample volume. 
Table 3-5. Porosity of Vertical Pour Samples.

\begin{tabular}{|c|c|c|}
\hline $\begin{array}{c}\text { Pour Height } \\
\text { (in.) }\end{array}$ & Sample ID & $\begin{array}{c}\text { Porosity } \\
\text { (\%) }\end{array}$ \\
\hline 0.5 & $0.5-10$ & 55.1 \\
\hline 1 & $1.0-10$ & 54.3 \\
\hline 3 & $3.0-10$ & 55.3 \\
\hline 6 & $6.0-10$ & 54.2 \\
\hline
\end{tabular}

\subsection{Conclusions}

The larger size of the planned SDU6 could result in saltstone being placed in thinner lifts as the unit is filled. This study was performed to determine whether thinner layers of saltstone negatively impact the performance of the waste form. A larger number of cold joints could potentially result in increased drying, salt deposition, and surface oxidation. A matrix of samples was prepared in 3 inch by 6 inch cylindrical molds to simulate thin pours ranging from 0.5 to 6 inches thick. These consisted of one lift (i.e., a full mold with no cold joints) up to twelve lifts (i.e., a full mold containing eleven cold joints) with the long axis of the cylindrical molds oriented both vertically and horizontally. Each sample was cured for at least 28 days prior to further characterization.

Leachability results showed that there is no obvious impact of the number of grout layers on the Leachability Index values for $\mathrm{Na}$ and $\mathrm{NO}_{3}$. The concentrations of $\mathrm{Cr}, \mathrm{NO}_{2}$, and $\mathrm{C}_{2} \mathrm{O}_{4}$ were below detection limits for all of the leachates. Since the $\mathrm{Cr}$ concentrations were all below the detection limit, no correlation was identified between leaching of $\mathrm{Cr}$ and $\mathrm{NO}_{3}$. Chromium is chemically stabilized in saltstone, while $\mathrm{NO}_{3}$ is assumed to be completely soluble. No attempt was made to evaluate the oxidation of these samples since no measureable $\mathrm{Cr}$ was leached, although this would appear to indicate that $\mathrm{Cr}$ in the samples remained reduced for cold joints with surfaces exposed for approximately four days.

The results of hydraulic conductivity measurements showed that the number of cold joints in the samples did not have a significant impact on the measured values for the vertical lift orientation (i.e., when the flow path is perpendicular to the cold joints). For the horizontal lift orientation (i.e., when the flow path is parallel to the cold joints), the number of cold joints in the samples also did not appear to have a significant impact on hydraulic conductivity. The measured hydraulic conductivity was faster when the flow path was parallel to the cold joints as compared to when the flow path was perpendicular to the cold joints. Percolation testing showed increased flow when the number of cold joints was increased.

Compressive strength testing showed that the maximum load at the onset of cracking was reduced by approximately $26 \%$ for those samples that contained cold joints (i.e., those with $0.5,1$, and 3 inch pour heights) as compared to the monolithic samples (i.e., the 6 inch pour height). The number of cold joints in the sample had no significant impact on the maximum load prior to cracking.

The porosity of the samples was not influenced by cold joints. This result was expected as the porosity is a material property affected by the properties of the components (premix and salt solution) and the water to premix ratio.

Overall, the only obvious impact of cold joints in the samples was to significantly increase hydraulic conductivity in the direction parallel to the cold joints. An increasing number of cold 
joints (thin layers) in the simulated saltstone samples did not exacerbate this effect, nor did it have a negative impact on the Leachability Indices or porosity for surfaces exposed for approximately four days. The presence of a cold joint reduced the compressive strength of the material, although this impact was seen regardless of the number of cold joints in the sample. An increasing number of thin layers did not further reduce the compressive strength, and this property is not considered in assessing the predicted performance of saltstone.

\subsection{Future Work}

The cold joints examined in this study cured for three to four days between pours. Longer times may have impacts on leachability due to surface oxidation and the deposition of salts resulting from drying. Future experiments could be tailored to determine whether the exposure time of the cold joints has a significant impact on saltstone properties. 
SRNL-STI-2012-00522

Revision 0

\subsection{References}

1. Baughman, T. C., "Saltstone Facility Disposal Unit \#6 Project, Bldg. 451-006Z (U)," U.S. Department of Energy Document M-TC-Z-00008, Revision 1, Savannah River Remediation, Aiken, SC (2012).

2. Wagnon, T. J., “Saltstone Disposal Unit (SDU) 6 Pre Mortem,” U.S. Department of Energy Document SRR-LWP-2011-00058, Savannah River Remediation, Aiken, SC (2011).

3. Brooks, T. E., "Saltstone Disposal Unit 6 Technology Development Program Plan,” U.S. Department of Energy Document G-TDP-Z-00001, Revision 0, Savannah River Remediation, Aiken, SC (2012).

4. Brooks, T. E., "Evaluate/Recommend Saltstone Grout and Placement Technical Issues for SDU\#6," U.S. Department of Energy Document G-TTR-Z-00001, Savannah River Remediation, Aiken, SC (2012).

5. Cozzi, A. D. and C. A. Langton, "Task Technical and Quality Assurance Plan for Task 3, Evaluation of the Impact of Thin Pours on Saltstone Properties,” U.S. Department of Energy Report SRNL-RP-2012-00266, Revision 0, Savannah River National Laboratory, Aiken, SC (2012).

6. "Specification of Procurement of Portland Type II Cement for the SPF (U)," U.S. Department of Energy Document X-SPP-Z-0004, Revision 3, Savannah River Remediation,

7. "Specification of Procurement of Slag for the SPF (U)," U.S. Department of Energy Document $X$-SPP-Z-0003, Revision 2, Savannah River Remediation,

8. "Specification of Procurement of Thermally Beneficiated Class F Fly Ash for the SPF (U)," U.S. Department of Energy Document X-SPP-Z-0002, Revision 3, Savannah River Remediation,

9. Reigel, M. M., "Results for the First Quarter 2011 Tank 50 WAC Slurry Sample: Chemical and Radionuclide Contaminant Results," U.S. Department of Energy Report SRNL-STI-201100030, Revision 0, Savannah River National Laboratory, Aiken, SC (2011).

10. Pickenheim, B. R. and M. M. Reigel, "Task Technical and Quality Assurance Plan for Properties of Various Water to Premix Ratios," U.S. Department of Energy Report SRNL-RP2012-00025, Revision 0, Savannah River National Laboratory, Aiken, SC (2012).

11. "Performance Assessment for the Saltstone Disposal Facility at the Savannah River Site," U.S. Department of Energy Report SRR-CWDA-2009-00017, Revision 0, (2009).

12. ANSI/ANS, "Measurement of the Leachability of Solidified Low-Level Radioactive Wastes by a Short-Term Test Procedure,” ANSI-ANS 16.1, (2008).

13. Flach, G. P., J. M. Jordan, and T. Whiteside, "Numerical Flow and Transport Simulations Supporting the Saltstone Disposal Facility Performance Assessment,” U.S. Department of Energy Report SRNL-STI-2009-00115, Revision 0, Savannah River National Laboratory, Aiken, SC (2009). 
14. ASTM, "Standard Test Methods for Measurement of Hydraulic Conductivity of Saturated Porous Materials Using a Flexible Wall Permeameter,” ASTM D-5084, (2003).

15. ASTM, "Standard Test Method for Field Measurement of Hydraulic Conductivity Using Borehole Infiltration,” ASTM D-6391, (2011).

16. ASTM, "Standard Test Method for Compressive Strength of Cylindrical Concrete Specimens,” ASTM C39/39M, (2010).

17. Harbour, J. R., V. J. Williams, T. B. Edwards, R. E. Eibling, and R. F. Schumacher, "Saltstone Variability Study - Measurement of Porosity," U.S. Department of Energy Report WSRC-STI-2007-00352, Rev. 0, Savannah River National Laboratory, Aiken, SC (2007).

18. Almond, P. M., D. I. Kaplan, C. A. Langton, D. B. Stefanko, W. A. Spencer, A. Hatfield, and Y. Arai, "Method Evaluation and Field Sample Measurements For The Rate of Movement of the Oxidation Front in Saltstone," U.S. Department of Energy Report SRNL-STI-2012-00468, Rev. 0, Savannah River National Laboratory, Aiken, SC (2012). 
SRNL-STI-2012-00522

Revision 0

Appendix A. Measurements from the Leachability Experiments. 
Table A-1. Measured Concentration of Each Contaminant, Conductivity, and pH for Each Leachate Aliquot after Each Interval for Each Sample.

\begin{tabular}{|c|c|c|c|c|c|c|c|c|c|c|}
\hline 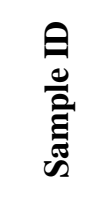 & 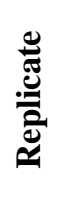 & 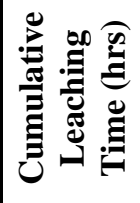 & $\begin{array}{c}\mathrm{Cr} \\
(\mathrm{mg} / \mathrm{L})\end{array}$ & $\begin{array}{c}\mathrm{Na} \\
(\mathrm{mg} / \mathrm{L})\end{array}$ & $\begin{array}{c}\text { Re } \\
(\mathrm{mg} / \mathrm{L})\end{array}$ & $\begin{array}{c}\mathrm{NO}_{2} \\
(\mathrm{mg} / \mathrm{L})\end{array}$ & $\begin{array}{c}\mathrm{NO}_{3} \\
(\mathrm{mg} / \mathrm{L})\end{array}$ & $\begin{array}{c}\mathrm{C}_{2} \mathrm{O}_{4} \\
(\mathrm{mg} / \mathrm{L})\end{array}$ & 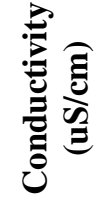 & pH \\
\hline $0.5-13$ & 1 & $30 \mathrm{~s}$ & $<0.100$ & 9.45 & $<0.040$ & $<10.0$ & 12.3 & $<10.0$ & 66.6 & 10.1 \\
\hline $0.5-13$ & 2 & $30 s$ & $<0.100$ & 9.39 & $<0.040$ & - & - & - & - & - \\
\hline $0.5-5$ & 1 & $30 s$ & $<0.100$ & 15 & 0.047 & $<10.0$ & 22.3 & $<10.0$ & 111 & 10.3 \\
\hline $0.5-5$ & 2 & $30 s$ & $<0.100$ & 15.2 & 0.051 & - & - & - & - & - \\
\hline $0.5-6$ & 1 & $30 s$ & $<0.100$ & 10.7 & $<0.040$ & $<10.0$ & 14.1 & $<10.0$ & 66.1 & 9.89 \\
\hline $0.5-6$ & 2 & $30 s$ & $<0.100$ & 10.9 & $<0.040$ & - & - & - & - & - \\
\hline $1.0-4$ & 1 & $30 s$ & $<0.100$ & 12.93 & 0.0427 & $<10.0$ & 13.3 & $<10.0$ & 67 & 7.76 \\
\hline $1.0-4$ & 2 & $30 s$ & $<0.100$ & 12.93 & 0.0466 & - & - & - & - & - \\
\hline $1.0-5$ & 1 & $30 s$ & $<0.100$ & 11.94 & 0.0414 & $<10.0$ & 11.6 & $<10.0$ & 70 & 9.81 \\
\hline $1.0-5$ & 2 & $30 s$ & $<0.100$ & 12.16 & 0.0427 & - & - & - & - & - \\
\hline $1.0-6$ & 1 & $30 s$ & $<0.100$ & 11.65 & 0.0447 & $<10.0$ & 11.7 & $<10.0$ & 76.3 & 10.6 \\
\hline $1.0-6$ & 2 & $30 s$ & $<0.100$ & 12.07 & 0.0479 & - & - & - & - & - \\
\hline $3.0-4$ & 1 & $30 s$ & $<0.100$ & 11.59 & 0.0445 & $<10.0$ & 13.2 & $<10.0$ & 72.7 & 10 \\
\hline $3.0-4$ & 2 & $30 s$ & $<0.100$ & 11.72 & 0.0467 & - & - & - & - & - \\
\hline $3.0-5$ & 1 & $30 s$ & $<0.100$ & 11.34 & 0.0459 & $<10.0$ & 12.1 & $<10.0$ & 69.9 & 9.93 \\
\hline $3.0-5$ & 2 & $30 \mathrm{~s}$ & $<0.100$ & 11.71 & 0.045 & - & - & - & - & - \\
\hline $3.0-6$ & 1 & $30 s$ & $<0.100$ & 15.42 & 0.0548 & $<10.0$ & 17.16 & $<10.0$ & 96.3 & 10.103 \\
\hline $3.0-6$ & 2 & $30 \mathrm{~s}$ & $<0.100$ & 15.68 & 0.0567 & - & - & - & - & - \\
\hline $6.0-4$ & 1 & $30 \mathrm{~s}$ & $<0.100$ & 8.59 & 0.044 & $<10.0$ & 14.7 & $<10.0$ & 56.4 & 9.07 \\
\hline $6.0-4$ & 2 & $30 \mathrm{~s}$ & $<0.100$ & 8.73 & 0.045 & - & - & - & - & - \\
\hline $6.0-5$ & 1 & $30 \mathrm{~s}$ & $<0.100$ & 9.79 & 0.046 & $<10.0$ & 16.4 & $<10.0$ & 67.8 & 9.16 \\
\hline $6.0-5$ & 2 & $30 s$ & $<0.100$ & 9.93 & 0.045 & - & - & - & - & - \\
\hline $6.0-6$ & 1 & $30 s$ & $<0.100$ & 8.93 & 0.045 & $<10.0$ & 13.8 & $<10.0$ & 50.5 & 8.54 \\
\hline $6.0-6$ & 2 & $30 s$ & $<0.100$ & 8.75 & 0.044 & - & - & - & - & - \\
\hline $0.5-13$ & 1 & 2 & $<0.100$ & 17.8 & 0.044 & $<10.0$ & 18.4 & $<10.0$ & 152 & 11 \\
\hline $0.5-13$ & 2 & 2 & $<0.100$ & 18.2 & 0.043 & - & - & - & - & - \\
\hline $0.5-5$ & 1 & 2 & $<0.100$ & 16.3 & 0.044 & $<10.0$ & 17.9 & $<10.0$ & 138 & 10.8 \\
\hline $0.5-5$ & 2 & 2 & $<0.100$ & 16.2 & 0.048 & - & - & - & - & - \\
\hline $0.5-6$ & 1 & 2 & $<0.100$ & 19.5 & 0.055 & $<10.0$ & 23.3 & $<10.0$ & 159 & 10.8 \\
\hline $0.5-6$ & 2 & 2 & $<0.100$ & 19.3 & 0.056 & - & - & - & - & - \\
\hline $1.0-4$ & 1 & 2 & $<0.100$ & 37.81 & 0.0999 & $<10.0$ & 44.4 & $<10.0$ & 285.1 & 10.8 \\
\hline $1.0-4$ & 2 & 2 & $<0.100$ & 38.55 & 0.0962 & - & - & - & - & - \\
\hline $1.0-5$ & 1 & 2 & $<0.100$ & 19.5 & 0.05599 & $<10.0$ & 44.7 & $<10.0$ & 137.5 & 10.49 \\
\hline $1.0-5$ & 2 & 2 & $<0.100$ & 19.23 & 0.0547 & - & - & - & - & - \\
\hline $1.0-6$ & 1 & 2 & $<0.100$ & 23 & 0.0628 & $<10.0$ & 24.2 & $<10.0$ & 155.8 & 10.5 \\
\hline $1.0-6$ & 2 & 2 & $<0.100$ & 23.45 & 0.0642 & - & - & - & - & - \\
\hline $3.0-4$ & 1 & 2 & $<0.100$ & 20.06 & 0.0543 & $<10.0$ & 19.3 & $<10.0$ & 148.3 & 10.52 \\
\hline $3.0-4$ & 2 & 2 & $<0.100$ & 20.15 & 0.0542 & - & - & - & - & - \\
\hline $3.0-5$ & 1 & 2 & $<0.100$ & 31.42 & 0.0823 & $<10.0$ & 33.9 & $<10.0$ & 222.6 & 10.67 \\
\hline $3.0-5$ & 2 & 2 & $<0.100$ & 31.59 & 0.0805 & - & - & - & - & - \\
\hline $3.0-6$ & 1 & 2 & $<0.100$ & 42.42 & 0.0982 & $<10.0$ & 47.7 & $<10.0$ & 302 & 10.81 \\
\hline
\end{tabular}


Table A-1. Measured Concentration of Each Contaminant, Conductivity, and pH for Each Leachate Aliquot after Each Interval for Each Sample. (cont'd)

\begin{tabular}{|c|c|c|c|c|c|c|c|c|c|c|}
\hline 会 & 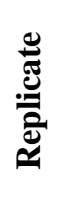 & 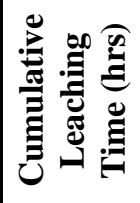 & $\underset{(\mathrm{mg} / \mathrm{L})}{\mathrm{Cr}}$ & $\begin{array}{c}\mathrm{Na} \\
(\mathrm{mg} / \mathrm{L})\end{array}$ & $\begin{array}{c}\operatorname{Re} \\
(\mathrm{mg} / \mathrm{L})\end{array}$ & $\begin{array}{c}\mathrm{NO}_{2} \\
(\mathrm{mg} / \mathrm{L})\end{array}$ & $\begin{array}{c}\mathrm{NO}_{3} \\
(\mathrm{mg} / \mathrm{L})\end{array}$ & $\begin{array}{c}\mathrm{C}_{2} \mathrm{O}_{4} \\
(\mathrm{mg} / \mathrm{L})\end{array}$ & 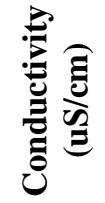 & pH \\
\hline $3.0-6$ & 2 & 2 & $<0.100$ & 43.2 & 0.0956 & - & - & - & - & - \\
\hline $6.0-4$ & 1 & 2 & $<0.100$ & 22.3 & 0.069 & $<10.0$ & 27.4 & $<10.0$ & 176.2 & 10.09 \\
\hline $6.0-4$ & 2 & 2 & $<0.100$ & 22.2 & 0.072 & - & - & - & - & - \\
\hline $6.0-5$ & 1 & 2 & $<0.100$ & 25.8 & 0.077 & $<10.0$ & 33.9 & $<10.0$ & 197.5 & 10.13 \\
\hline $6.0-5$ & 2 & 2 & $<0.100$ & 26.1 & 0.077 & - & - & - & - & - \\
\hline $6.0-6$ & 1 & 2 & $<0.100$ & 21.4 & 0.07 & $<10.0$ & 26.8 & $<10.0$ & 168 & 9.99 \\
\hline $6.0-6$ & 2 & 2 & $<0.100$ & 21.3 & 0.069 & - & - & - & - & - \\
\hline $0.5-13$ & 1 & 7 & $<0.100$ & 23.3 & 0.071 & $<10.0$ & 27.2 & $<10.0$ & 200 & 11 \\
\hline $0.5-13$ & 2 & 7 & $<0.100$ & 23.3 & 0.07 & - & - & - & - & - \\
\hline $0.5-5$ & 1 & 7 & $<0.100$ & 17.7 & 0.059 & $<10.0$ & 22.5 & $<10.0$ & 157 & 10.8 \\
\hline $0.5-5$ & 2 & 7 & $<0.100$ & 17.9 & 0.058 & - & - & - & - & - \\
\hline $0.5-6$ & 1 & 7 & $<0.100$ & 20.7 & 0.065 & $<10.0$ & 25.2 & $<10.0$ & 182 & 10.8 \\
\hline $0.5-6$ & 2 & 7 & $<0.100$ & 21.4 & 0.067 & - & - & - & - & - \\
\hline $1.0-4$ & 1 & 7 & $<0.100$ & 19.34 & 0.0543 & $<10.0$ & 19.1 & $<10.0$ & 150.1 & 10.52 \\
\hline $1.0-4$ & 2 & 7 & $<0.100$ & 19.51 & 0.0586 & - & - & - & - & - \\
\hline $1.0-5$ & 1 & 7 & $<0.100$ & 22.88 & 0.062 & $<10.0$ & 25.1 & $<10.0$ & 115.3 & 8.32 \\
\hline $1.0-5$ & 2 & 7 & $<0.100$ & 22.59 & 0.0576 & - & - & - & - & - \\
\hline $1.0-6$ & 1 & 7 & $<0.100$ & 27.03 & 0.0718 & $<10.0$ & 31 & $<10.0$ & 209.2 & 10.62 \\
\hline $1.0-6$ & 2 & 7 & $<0.100$ & 27.16 & 0.0736 & - & - & - & - & - \\
\hline $3.0-4$ & 1 & 7 & $<0.100$ & 20.33 & 0.0541 & $<10.0$ & 19.8 & $<10.0$ & 103.2 & 8.43 \\
\hline $3.0-4$ & 2 & 7 & $<0.100$ & 20.42 & 0.0546 & - & - & - & - & - \\
\hline $3.0-5$ & 1 & 7 & $<0.100$ & 25.31 & 0.0685 & $<10.0$ & 25.3 & $<10.0$ & 127.9 & 8.3 \\
\hline $3.0-5$ & 2 & 7 & $<0.100$ & 25.29 & 0.0666 & - & - & - & - & - \\
\hline $3.0-6$ & 1 & 7 & $<0.100$ & 35.35 & 0.0887 & $<10.0$ & 37.47 & $<10.0$ & 231.6 & 10.39 \\
\hline $3.0-6$ & 2 & 7 & $<0.100$ & 35.76 & 0.0872 & - & - & - & - & - \\
\hline $6.0-4$ & 1 & 7 & $<0.100$ & 39.3 & 0.099 & $<10.0$ & 48.8 & $<10.0$ & 340 & 10.35 \\
\hline $6.0-4$ & 2 & 7 & $<0.100$ & 39.2 & 0.1 & - & - & - & - & - \\
\hline $6.0-5$ & 1 & 7 & $<0.100$ & 39.4 & 0.108 & $<10.0$ & 50 & $<10.0$ & 342 & 10.33 \\
\hline $6.0-5$ & 2 & 7 & $<0.100$ & 39.2 & 0.108 & - & - & - & - & - \\
\hline $6.0-6$ & 1 & 7 & $<0.100$ & 23.7 & 0.068 & $<10.0$ & 30.7 & $<10.0$ & 211.5 & 10.08 \\
\hline $6.0-6$ & 2 & 7 & $<0.100$ & 23.4 & 0.068 & - & - & - & - & - \\
\hline $0.5-13$ & 1 & 24 & $<0.100$ & 39.2 & 0.107 & $<10.0$ & 47 & $<10.0$ & 331 & 11.2 \\
\hline $0.5-13$ & 2 & 24 & $<0.100$ & 39.4 & 0.109 & - & - & - & - & - \\
\hline $0.5-5$ & 1 & 24 & $<0.100$ & 47 & 0.127 & $<10.0$ & 60.6 & $<10.0$ & 393 & 11.2 \\
\hline $0.5-5$ & 2 & 24 & $<0.100$ & 46.9 & 0.129 & - & - & - & - & - \\
\hline $0.5-6$ & 1 & 24 & $<0.100$ & 42.8 & 0.113 & $<10.0$ & 53.9 & $<<10.0$ & 343 & 11.2 \\
\hline $0.5-6$ & 2 & 24 & $<0.100$ & 43 & 0.115 & - & - & - & - & - \\
\hline $1.0-4$ & 1 & 24 & $<0.100$ & 62.4 & 0.1479 & $<10.0$ & 75.1 & $<10.0$ & 492 & 11.01 \\
\hline $1.0-4$ & 2 & 24 & $<0.100$ & 63.1 & 0.1468 & - & - & - & - & - \\
\hline $1.0-5$ & 1 & 24 & $<0.100$ & 42.7 & 0.1042 & $<10.0$ & 46.8 & $<10.0$ & 340 & 10.89 \\
\hline $\begin{array}{l}1.0-5 \\
\end{array}$ & 2 & 24 & $<0.100$ & 41.95 & 0.1065 & - & - & - & - & - \\
\hline $1.0-6$ & 1 & 24 & $<0.100$ & 30.9 & 0.075 & $<10.0$ & 34.1 & $<10.0$ & 241.7 & 10.75 \\
\hline
\end{tabular}


Table A-1. Measured Concentration of Each Contaminant, Conductivity, and pH for Each Leachate Aliquot after Each Interval for Each Sample. (cont'd)

\begin{tabular}{|c|c|c|c|c|c|c|c|c|c|c|}
\hline 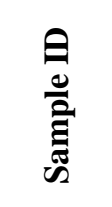 & 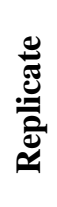 & 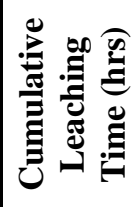 & $\underset{(\mathrm{mg} / \mathrm{L})}{\mathrm{Cr}}$ & $\begin{array}{c}\mathrm{Na} \\
(\mathrm{mg} / \mathrm{L})\end{array}$ & $\begin{array}{c}\mathrm{Re} \\
(\mathrm{mg} / \mathrm{L})\end{array}$ & $\begin{array}{c}\mathrm{NO}_{2} \\
(\mathrm{mg} / \mathrm{L})\end{array}$ & $\begin{array}{c}\mathrm{NO}_{3} \\
(\mathrm{mg} / \mathrm{L})\end{array}$ & $\begin{array}{c}\mathrm{C}_{2} \mathrm{O}_{4} \\
(\mathrm{mg} / \mathrm{L})\end{array}$ & 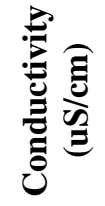 & pH \\
\hline $1.0-6$ & 2 & 24 & $<0.100$ & 31.4 & 0.0747 & - & - & - & - & - \\
\hline $3.0-4$ & 1 & 24 & $<0.100$ & 69.85 & 0.1419 & $<10.0$ & 75.6 & $<10.0$ & 526 & 11.11 \\
\hline $3.0-4$ & 2 & 24 & $<0.100$ & 70.2 & 0.1397 & - & - & - & - & - \\
\hline $3.0-5$ & 1 & 24 & $<0.100$ & 31.74 & 0.0738 & $<10.0$ & 32.9 & $<10.0$ & 256.1 & 10.71 \\
\hline $3.0-5$ & 2 & 24 & $<0.100$ & 32.75 & 0.076 & - & - & - & - & - \\
\hline $3.0-6$ & 1 & 24 & $<0.100$ & 33.49 & 0.0775 & $<10.0$ & 34.4 & $<10.0$ & 270.4 & 10.71 \\
\hline $3.0-6$ & 2 & 24 & $<0.100$ & 33.43 & 0.075 & - & - & - & - & - \\
\hline $6.0-4$ & 1 & 24 & $<0.100$ & 59.9 & 0.143 & $<10.0$ & 71.6 & $<10.0$ & 543 & 10.55 \\
\hline $6.0-4$ & 2 & 24 & $<0.100$ & 60 & 0.139 & - & - & - & - & - \\
\hline $6.0-5$ & 1 & 24 & $<0.100$ & 63.4 & 0.158 & $<10.0$ & 76.2 & $<10.0$ & 544 & 10.6 \\
\hline $6.0-5$ & 2 & 24 & $<0.100$ & 62.9 & 0.155 & - & - & - & - & - \\
\hline $6.0-6$ & 1 & 24 & $<0.100$ & 25.8 & 0.075 & $<10.0$ & 29.3 & $<10.0$ & 236 & 10.22 \\
\hline $6.0-6$ & 2 & 24 & $<0.100$ & 26 & 0.074 & - & - & - & - & - \\
\hline $0.5-13$ & 1 & 48 & $<0.100$ & 35.4 & 0.088 & $<10.0$ & 41.1 & $<<10.0$ & 304 & 11 \\
\hline $0.5-13$ & 2 & 48 & $<0.100$ & 35.1 & 0.088 & - & - & - & - & - \\
\hline $0.5-5$ & 1 & 48 & $<0.100$ & 27.3 & 0.073 & $<10.0$ & 31.2 & $<10.0$ & 231 & 11 \\
\hline $0.5-5$ & 2 & 48 & $<0.100$ & 26.5 & 0.079 & - & - & - & - & - \\
\hline $0.5-6$ & 1 & 48 & $<0.100$ & 44.2 & 0.116 & $<10.0$ & 54.7 & $<10.0$ & 377 & 11.1 \\
\hline $0.5-6$ & 2 & 48 & $<0.100$ & 44.5 & 0.115 & - & - & - & - & - \\
\hline $1.0-4$ & 1 & 48 & $<0.100$ & 26.62 & 0.0693 & $<10.0$ & 27.8 & $<10.0$ & 233.7 & 10.66 \\
\hline $1.0-4$ & 2 & 48 & $<0.100$ & 26.51 & 0.0713 & - & - & - & - & - \\
\hline $1.0-5$ & 1 & 48 & $<0.100$ & 44.7 & 0.1029 & $<10.0$ & 50.4 & $<10.0$ & 397 & 10.9 \\
\hline $1.0-5$ & 2 & 48 & $<0.100$ & 45 & 0.1019 & - & - & - & - & - \\
\hline $1.0-6$ & 1 & 48 & $<0.100$ & 32.09 & 0.0822 & $<10.0$ & 32.8 & $<10.0$ & 237 & 10.55 \\
\hline $1.0-6$ & 2 & 48 & $<0.100$ & 31.46 & 0.0808 & - & - & - & - & - \\
\hline $3.0-4$ & 1 & 48 & $<0.100$ & 24.6 & 0.0634 & $<10.0$ & 22.6 & $<10.0$ & 218.8 & 10.54 \\
\hline $3.0-4$ & 2 & 48 & $<0.100$ & 24.2 & 0.0607 & - & - & - & - & - \\
\hline $3.0-5$ & 1 & 48 & $<0.100$ & 45.38 & 0.1079 & $<10.0$ & 23.9 & $<10.0$ & 390 & 10.83 \\
\hline $3.0-5$ & 2 & 48 & $<0.100$ & 46 & 0.1052 & - & - & - & - & - \\
\hline $3.0-6$ & 1 & 48 & $<0.100$ & 54.6 & 0.1283 & $<10.0$ & 56.47 & $<10.0$ & 453 & 10.92 \\
\hline $3.0-6$ & 2 & 48 & $<0.100$ & 54.24 & 0.1269 & - & - & - & - & - \\
\hline $6.0-4$ & 1 & 48 & $<0.100$ & 24.9 & 0.064 & $<10.0$ & 28.3 & $<10.0$ & 236.5 & 10.16 \\
\hline $6.0-4$ & 2 & 48 & $<0.100$ & 24.8 & 0.065 & - & - & - & - & - \\
\hline $6.0-5$ & 1 & 48 & $<0.100$ & 45 & 0.111 & $<10.0$ & 53 & $<10.0$ & 426 & 10.48 \\
\hline $6.0-5$ & 2 & 48 & $<0.100$ & 45.4 & 0.11 & - & - & - & - & - \\
\hline $6.0-6$ & 1 & 48 & $<0.100$ & 23.4 & 0.066 & $<10.0$ & 24.6 & $<10.0$ & 223.3 & 10.21 \\
\hline $6.0-6$ & 2 & 48 & $<0.100$ & 23.5 & 0.065 & - & - & - & - & - \\
\hline $0.5-13$ & 1 & 72 & $<0.100$ & 16.1 & 0.046 & $<10.0$ & 17.1 & $<10.0$ & 145 & 10.9 \\
\hline $0.5-13$ & 2 & 72 & $<0.100$ & 15.1 & 0.046 & - & - & - & - & - \\
\hline $0.5-5$ & 1 & 72 & $<0.100$ & 16.6 & 0.051 & $<10.0$ & 17.7 & $<10.0$ & 154 & 10.9 \\
\hline $\begin{array}{c}0.5-5 \\
\end{array}$ & 2 & 72 & $<0.100$ & 16.3 & 0.053 & - & - & - & - & - \\
\hline $0.5-6$ & 1 & 72 & $<0.100$ & 27.2 & 0.077 & $<10.0$ & 30.3 & $<10.0$ & 251 & 11.1 \\
\hline
\end{tabular}


Table A-1. Measured Concentration of Each Contaminant, Conductivity, and pH for Each Leachate Aliquot after Each Interval for Each Sample. (cont'd)

\begin{tabular}{|c|c|c|c|c|c|c|c|c|c|c|}
\hline 会 & 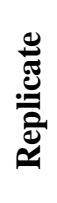 & 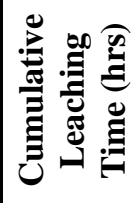 & $\underset{(\mathrm{mg} / \mathrm{L})}{\mathrm{Cr}}$ & $\begin{array}{c}\mathrm{Na} \\
(\mathrm{mg} / \mathrm{L})\end{array}$ & $\begin{array}{c}\operatorname{Re} \\
(\mathrm{mg} / \mathrm{L})\end{array}$ & $\begin{array}{c}\mathrm{NO}_{2} \\
(\mathrm{mg} / \mathrm{L})\end{array}$ & $\begin{array}{c}\mathrm{NO}_{3} \\
(\mathrm{mg} / \mathrm{L})\end{array}$ & $\begin{array}{c}\mathrm{C}_{2} \mathrm{O}_{4} \\
(\mathrm{mg} / \mathrm{L})\end{array}$ & 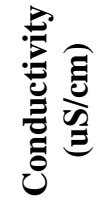 & pH \\
\hline $0.5-6$ & 2 & 72 & $<0.100$ & 28 & 0.078 & - & - & - & - & - \\
\hline $1.0-4$ & 1 & 72 & $<0.100$ & 34.9 & 0.0856 & $<10.0$ & 34.4 & $<10.0$ & 320 & 10.9 \\
\hline $1.0-4$ & 2 & 72 & $<0.100$ & 34.1 & 0.0857 & - & - & - & - & - \\
\hline $1.0-5$ & 1 & 72 & $<0.100$ & 20.84 & 0.055 & $<10.0$ & 17.2 & $<10.0$ & 185.7 & 10.72 \\
\hline $1.0-5$ & 2 & 72 & $<0.100$ & 21.15 & 0.0505 & - & - & - & - & - \\
\hline $1.0-6$ & 1 & 72 & $<0.100$ & 24.57 & 0.0609 & $<10.0$ & 21.26 & $<10.0$ & 212.1 & 10.8 \\
\hline $1.0-6$ & 2 & 72 & $<0.100$ & 24.72 & 0.0597 & - & - & - & - & - \\
\hline $3.0-4$ & 1 & 72 & $<0.100$ & 18.26 & 0.0439 & $<10.0$ & 14.2 & $<10.0$ & 167.6 & 10.66 \\
\hline $3.0-4$ & 2 & 72 & $<0.100$ & 18.34 & 0.0444 & - & - & - & - & - \\
\hline $3.0-5$ & 1 & 72 & $<0.100$ & 26.4 & 0.064 & $<10.0$ & 24.9 & $<10.0$ & 244.8 & 10.84 \\
\hline $3.0-5$ & 2 & 72 & $<0.100$ & 26.51 & 0.0629 & - & - & - & - & - \\
\hline $3.0-6$ & 1 & 72 & $<0.100$ & 20.59 & 0.0484 & $<10.0$ & 16.27 & $<10.0$ & 163.4 & 10.64 \\
\hline $3.0-6$ & 2 & 72 & $<0.100$ & 20.7 & 0.0489 & - & - & - & - & - \\
\hline $6.0-4$ & 1 & 72 & $<0.100$ & 19.2 & 0.054 & $<10.0$ & 19.8 & $<<10.0$ & 200 & 10.16 \\
\hline $6.0-4$ & 2 & 72 & $<0.100$ & 19 & 0.054 & - & - & - & - & - \\
\hline $6.0-5$ & 1 & 72 & $<0.100$ & 31.2 & 0.082 & $<10.0$ & 36.3 & $<10.0$ & 317 & 10.34 \\
\hline $6.0-5$ & 2 & 72 & $<0.100$ & 31.7 & 0.081 & - & - & - & - & - \\
\hline $6.0-6$ & 1 & 72 & $<0.100$ & 17.8 & 0.055 & $<10.0$ & 18.2 & $<10.0$ & 182.3 & 10.14 \\
\hline $6.0-6$ & 2 & 72 & $<0.100$ & 18 & 0.055 & - & - & - & - & - \\
\hline $0.5-13$ & 1 & 96 & $<0.100$ & 16.8 & 0.052 & $<10.0$ & 19.3 & $<10.0$ & 168 & 10.9 \\
\hline $0.5-13$ & 2 & 96 & $<0.100$ & 16.9 & 0.05 & - & - & - & - & - \\
\hline $0.5-5$ & 1 & 96 & $<0.100$ & 21.3 & 0.062 & $<10.0$ & 25.7 & $<10.0$ & 213 & 11 \\
\hline $0.5-5$ & 2 & 96 & $<0.100$ & 21.1 & 0.063 & - & - & - & - & - \\
\hline $0.5-6$ & 1 & 96 & $<0.100$ & 24.4 & 0.07 & $<10.0$ & 29.4 & $<10.0$ & 229 & 11 \\
\hline $0.5-6$ & 2 & 96 & $<0.100$ & 24.6 & 0.071 & - & - & - & - & - \\
\hline $1.0-4$ & 1 & 96 & $<0.100$ & 19.67 & 0.051 & $<10.0$ & 17.6 & $<10.0$ & 172.9 & 10.55 \\
\hline $1.0-4$ & 2 & 96 & $<0.100$ & 19.48 & 0.0566 & - & - & - & - & - \\
\hline $1.0-5$ & 1 & 96 & $<0.100$ & 18.95 & 0.047 & $<10.0$ & 15.4 & $<10.0$ & 173.5 & 10.53 \\
\hline $1.0-5$ & 2 & 96 & $<0.100$ & 19.3 & 0.0473 & - & - & - & - & - \\
\hline $1.0-6$ & 1 & 96 & $<0.100$ & 25.2 & 0.0602 & $<10.0$ & 21.26 & $<10.0$ & 221.6 & 10.63 \\
\hline $1.0-6$ & 2 & 96 & $<0.100$ & 24.77 & 0.0639 & - & - & - & - & - \\
\hline $3.0-4$ & 1 & 96 & $<0.100$ & 20.06 & 0.0506 & $<10.0$ & 17.4 & $<10.0$ & 183.9 & 10.51 \\
\hline $3.0-4$ & 2 & 96 & $<0.100$ & 19.91 & 0.0533 & - & - & - & - & - \\
\hline $3.0-5$ & 1 & 96 & $<0.100$ & 26.2 & 0.064 & $<10.0$ & 18 & $<10.0$ & 247.7 & 10.68 \\
\hline $3.0-5$ & 2 & 96 & $<0.100$ & 26.45 & 0.065 & - & - & - & - & - \\
\hline $3.0-6$ & 1 & 96 & $<0.100$ & 24.26 & 0.0568 & $<10.0$ & 20.7 & $<10.0$ & 219.5 & 10.63 \\
\hline $3.0-6$ & 2 & 96 & $<0.100$ & 24.19 & 0.0613 & - & - & - & - & - \\
\hline $6.0-4$ & 1 & 96 & $<0.100$ & 19.5 & 0.054 & $<10.0$ & 20.9 & $<10.0$ & 199.3 & 10.44 \\
\hline $6.0-4$ & 2 & 96 & $<0.100$ & 19.4 & 0.055 & - & - & - & - & - \\
\hline $6.0-5$ & 1 & 96 & $<0.100$ & 23.3 & 0.07 & $<10.0$ & 29.9 & $<10.0$ & 280.8 & 10.6 \\
\hline $6.0-5$ & 2 & 96 & $<0.100$ & 26.5 & 0.069 & - & - & - & - & - \\
\hline $6.0-6$ & 1 & 96 & $<0.100$ & 11.7 & 0.041 & $<10.0$ & 11.4 & $<10.0$ & 129.7 & 10.27 \\
\hline
\end{tabular}


Table A-1. Measured Concentration of Each Contaminant, Conductivity, and pH for Each Leachate Aliquot after Each Interval for Each Sample. (cont'd)

\begin{tabular}{|c|c|c|c|c|c|c|c|c|c|c|}
\hline 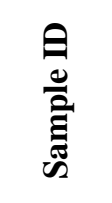 & 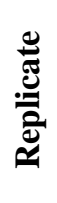 & 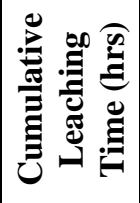 & $\underset{(\mathrm{mg} / \mathrm{L})}{\mathrm{Cr}}$ & $\begin{array}{c}\mathrm{Na} \\
(\mathrm{mg} / \mathrm{L})\end{array}$ & $\begin{array}{c}\operatorname{Re} \\
(\mathrm{mg} / \mathrm{L})\end{array}$ & $\begin{array}{c}\mathrm{NO}_{2} \\
(\mathrm{mg} / \mathrm{L})\end{array}$ & $\begin{array}{c}\mathrm{NO}_{3} \\
\text { (mg/L) }\end{array}$ & $\begin{array}{c}\mathrm{C}_{2} \mathrm{O}_{4} \\
(\mathrm{mg} / \mathrm{L})\end{array}$ & 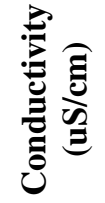 & pH \\
\hline $6.0-6$ & 2 & 96 & $<0.100$ & 11.6 & 0.04 & - & - & - & - & - \\
\hline $0.5-13$ & 1 & 120 & $<0.100$ & 9.63 & $<0.040$ & $<10.0$ & 10.7 & $<10.0$ & 94 & 10.5 \\
\hline $0.5-13$ & 2 & 120 & $<0.100$ & 10.7 & $<0.040$ & - & - & - & - & - \\
\hline $0.5-5$ & 1 & 120 & $<0.100$ & 15.8 & 0.048 & $<10.0$ & 16.8 & $<10.0$ & 162 & 10.8 \\
\hline $0.5-5$ & 2 & 120 & $<0.100$ & 15.7 & 0.047 & - & - & - & - & - \\
\hline $0.5-6$ & 1 & 120 & $<0.100$ & 23.9 & 0.067 & $<10.0$ & 27.3 & $<10.0$ & 230 & 11 \\
\hline $0.5-6$ & 2 & 120 & $<0.100$ & 24 & 0.069 & - & - & - & - & - \\
\hline $6.0-4$ & 1 & 120 & $<0.100$ & 28 & 0.073 & $<10.0$ & 28.9 & $<10.0$ & 269.9 & 10.48 \\
\hline $6.0-4$ & 2 & 120 & $<0.100$ & 28.3 & 0.072 & - & - & - & - & - \\
\hline $6.0-5$ & 1 & 120 & $<0.100$ & 25.5 & 0.067 & $<10.0$ & 25.5 & $<10.0$ & 223 & 10.38 \\
\hline $6.0-5$ & 2 & 120 & $<0.100$ & 25.4 & 0.066 & - & - & - & - & - \\
\hline $6.0-6$ & 1 & 120 & $<0.100$ & 25.5 & 0.066 & $<10.0$ & 24.6 & $<10.0$ & 229.7 & 10.35 \\
\hline $6.0-6$ & 2 & 120 & $<0.100$ & 26.5 & 0.068 & - & - & - & - & - \\
\hline $1.0-4$ & 1 & 168 & $<0.100$ & 59.96 & 0.1398 & $<10.0$ & 66.4 & $<10.0$ & 517 & 11.02 \\
\hline $1.0-4$ & 2 & 168 & $<0.100$ & 60.5 & 0.1374 & - & - & - & - & - \\
\hline $1.0-5$ & 1 & 168 & $<0.100$ & 35.69 & 0.0773 & $<10.0$ & 34 & $<10.0$ & 283.5 & 10.68 \\
\hline $1.0-5$ & 2 & 168 & $<0.100$ & 35.34 & 0.0807 & - & - & - & - & - \\
\hline $1.0-6$ & 1 & 168 & $<0.100$ & 33.77 & 0.0745 & $<10.0$ & 29.3 & $<10.0$ & 254.4 & 10.64 \\
\hline $1.0-6$ & 2 & 168 & $<0.100$ & 33.7 & 0.0714 & - & - & - & - & - \\
\hline $3.0-4$ & 1 & 168 & $<0.100$ & 41.46 & 0.0927 & $<10.0$ & 46.2 & $<10.0$ & 354 & 10.79 \\
\hline $3.0-4$ & 2 & 168 & $<0.100$ & 42.85 & 0.0907 & - & - & - & - & - \\
\hline $3.0-5$ & 1 & 168 & $<0.100$ & 44.68 & 0.0957 & $<10.0$ & 44.87 & $<10.0$ & 373 & 10.87 \\
\hline $3.0-5$ & 2 & 168 & $<0.100$ & 44.82 & 0.0982 & - & - & - & - & - \\
\hline $3.0-6$ & 1 & 168 & $<0.100$ & 51.79 & 0.1131 & $<10.0$ & 55.7 & $<10.0$ & 398 & 10.84 \\
\hline $3.0-6$ & 2 & 168 & $<0.100$ & 53.06 & 0.1065 & - & - & - & - & - \\
\hline
\end{tabular}


SRNL-STI-2012-00522

Revision 0

Appendix B. AMEC Hydraulic Conductivity Test Report. 


\section{$\operatorname{amec}^{\theta}$}

August 1, 2012

Savannah River Nuclear Solutions

Bldg. 730-2B Room 2158

Aiken, SC 29808

Attention: Mr. Bill Joyce, STR

Subject: $\quad$ Test Report - SDU-6 Task 3 Grout Samples

Subcontract No. AC54317N, Delivery Order No. 34

Specification K-SPC-G-00013, Rev. 13

AMEC Project No. 6155-08-0031

Dear Mr. Joyce:

AMEC Environment \& Infrastructure (AMEC) has completed the assigned testing services for Delivery Order No. 34, Subcontract No. AC54317N. The test results are included in Attachment 1. An equipment list used in this Delivery Order is included in Attachment 2. The tests performed in this Delivery Order are listed below along with applicable ASTM or other procedures:

Permeability

ASTM D5084

These tests were performed in accordance with the above referenced contract order and AMEC's Quality Assurance Manual (QAM) Revision 1.

We appreciate the opportunity of serving your geotechnical laboratory testing needs. If you have questions, please contact us.

Sincerely,
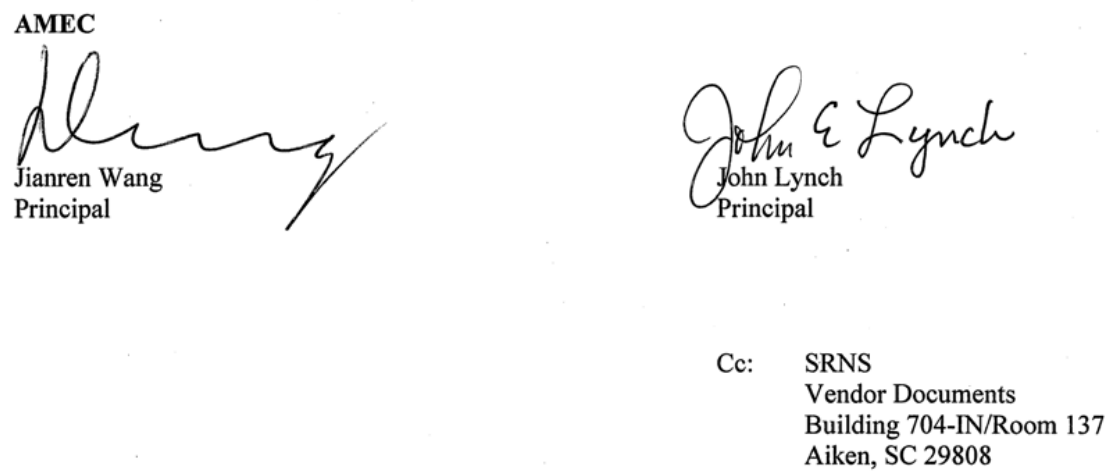

AMEC Environment \& Infrastructure

396 Plasters Avenue, NE • Atlanta, GA 30324 • Phone: 404-873-4761 • Fax: 404-817-0221

AMEC.com 
SRNL-STI-2012-00522

Revision 0

SDU-6 Task 3 Grout Samples AC54317N DO34 AMEC Project No. 6155-08-0031

August 1, 2012

\section{ATTACHMENT 1}

Page 2 of 3

RCN: SRS256

Page 2 of 46

B-3 


\section{amec $^{\circ}$}

\section{HYDRAULIC CONDUCTIVITY}

$\begin{array}{ll}\text { Project No. } & \text { 6155-08-0031.34 } \\ \text { Project Name } & \text { SDU-6 Task } 3 \text { Grout Samples } \\ \text { Boring No. } & \text { V6.0-1 } \\ \text { Sample No. } & \text { V6.0-1 } \\ \text { Sample Depth } & \text { N/A } \\ \text { Sample Description Grout Core }\end{array}$

Project No.

\author{
Tested By $\boldsymbol{J W} \boldsymbol{\&} \boldsymbol{L}$ \\ Test Date $\quad 6 / 15 / 2012$ \\ Reviewed By $J W$ OCG \\ Review Date $8 / 1 / 2012$ \\ Lab No. $\quad 11605$
}

ASTM D5084 - Method F (CVFH)

\begin{tabular}{|l|l|}
\hline Sample Type: & Core \\
\hline Sample Orientation: & Vertical \\
\hline Initial Water Content, \%: & 44.1 \\
\hline Wet Unit Weight, pcf: & 105.8 \\
\hline Dry Unit Weight, pcf: & 73.4 \\
\hline Compaction, \%: & N/A \\
\hline Hydraulic Conductivity, cm/sec. @20 ${ }^{\circ} \mathrm{C}$ & $\mathbf{5 . 9 E - 1 1}$ \\
\hline
\end{tabular}

Remarks: 
SRNL-STI-2012-00522

Revision 0

PERMEABILITY TEST

(ASTM D5084 - 03) (Method F, Constant Volume Falling Head)

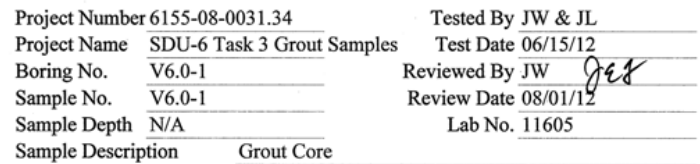

\begin{tabular}{|c|r|c|r|c|r|}
\hline \multicolumn{4}{|c|}{ Initial Sample Data } & \multicolumn{2}{c|}{ Final Sample Data } \\
\hline \multicolumn{2}{|c|}{ Length, in } & \multicolumn{2}{|c|}{ Diameter, in } & Pan No. & N/A \\
\hline Location 1 & 4.137 & Location 1 & 3.040 & Wet Soil+Pan, grams & 825.75 \\
\hline Location 2 & 4.159 & Location 2 & 3.023 & Dry Soil + Pan, grams & 572.66 \\
\hline Location3 & 4.087 & Location 3 & 3.021 & Pan Weight, grams & 0 \\
\hline Average & 4.128 & Average & 3.028 & Moisture Content, \% & 44.2 \\
\hline Volume, in & 29.72 & Wet Soil + Tare, grams & 825.13 & Dry Unit Weight, pcf & 73.4 \\
\hline SG Assumed & 2.40 & Tare Weight, grams & 0.00 & Saturation, \% & 101.9 \\
\hline Soil Sample Wt., g & 825.13 & Dry Soil +Tare, grams & 572.66 & Diameter, in. & N/A \\
\hline Dry UW, pcf & 73.4 & Moisture Content, \% & 44.1 & Length, in. & N/A \\
\hline Saturation, \% & 101.7 & & & Volume, in ${ }^{3}$ & N/A \\
\hline
\end{tabular}

\section{$\operatorname{amec}^{\theta}$}

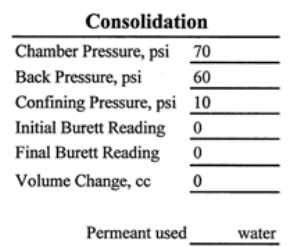

\begin{tabular}{|r|r|r|r|r|r|r|r|r|r|}
\hline $\begin{array}{c}\text { Elapsed Time } \\
(\mathrm{sec})\end{array}$ & $\begin{array}{c}\mathrm{z}_{\mathrm{o}} \\
(\mathrm{cm})\end{array}$ & $\begin{array}{c}\mathrm{za} \\
(\mathrm{cm})\end{array}$ & $\begin{array}{c}\mathrm{zb} \\
(\mathrm{cm})\end{array}$ & $\begin{array}{c}\Delta \mathrm{z}_{\mathrm{p}} \\
(\mathrm{cm})\end{array}$ & $\begin{array}{c}\text { Temp } \\
\left({ }^{\circ} \mathrm{C}\right)\end{array}$ & $\begin{array}{c}\text { Intial } \\
\text { Hydraulic } \\
\text { Gradient }\end{array}$ & $\begin{array}{c}\text { Final } \\
\text { Hydraulic } \\
\text { Gradient }\end{array}$ & $\begin{array}{c}\mathrm{k} \\
\mathrm{cm} / \mathrm{sec}\end{array}$ & $\begin{array}{c}\mathrm{k} \\
\mathrm{cm} / \mathrm{sec} \\
\mathrm{at} 20^{\circ} \mathrm{C}\end{array}$ \\
\hline 67440 & 1.70 & 27.10 & 26.90 & 0.20 & 23.6 & 30.5 & 30.2 & $6.61 \mathrm{E}-11$ & $6.07 \mathrm{E}-11$ \\
\hline 95520 & 1.70 & 27.10 & 26.85 & 0.25 & 22.3 & 30.5 & 30.1 & $5.84 \mathrm{E}-11$ & $5.53 \mathrm{E}-11$ \\
\hline 330660 & 1.70 & 25.70 & 24.90 & 0.80 & 22.6 & 28.8 & 27.8 & $5.79 \mathrm{E}-11$ & $5.44 \mathrm{E}-11$ \\
\hline 347580 & 1.70 & 25.70 & 24.80 & 0.90 & 22.6 & 28.8 & 27.7 & $6.21 \mathrm{E}-11$ & $5.83 \mathrm{E}-11$ \\
\hline 360000 & 1.70 & 25.70 & 24.75 & 0.95 & 22.6 & 28.8 & 27.6 & $6.33 \mathrm{E}-11$ & $5.95 \mathrm{E}-11$ \\
\hline 414021 & 1.70 & 25.70 & 24.50 & 1.20 & 22.3 & 28.8 & 27.3 & $6.99 \mathrm{E}-11$ & $6.62 \mathrm{E}-11$ \\
\hline & & & & & & & & & \\
\hline
\end{tabular}

\begin{tabular}{|c|c|c|c|c|}
\hline No. of Trials & $\begin{array}{c}\text { Sample } \\
\text { Type }\end{array}$ & $\begin{array}{c}\text { Max. Density } \\
(\mathrm{pcf})\end{array}$ & $\begin{array}{c}\text { Compaction } \\
\%\end{array}$ & $\begin{array}{c}\text { Sample } \\
\text { Orientation }\end{array}$ \\
\hline 6 & Core & N/A & N/A & Vertical \\
\hline
\end{tabular}

Avg. $\mathrm{k}$ at $20^{\circ} \mathrm{C} \quad \underline{5.9 \mathrm{E}-11} \mathrm{~cm} / \mathrm{sec}$

$$
\begin{aligned}
& \mathrm{a}_{\mathrm{2}}=\quad 0.76712 \mathrm{~cm}^{2} \\
& \mathrm{~A}=\quad-46.46 \mathrm{~cm}^{2} \\
& \mathrm{~L}=\quad 10.48 \mathrm{~cm}
\end{aligned}
$$

$S=L / A=0.225671 / \mathrm{cm}$

$\mathrm{a}_{\mathrm{p}}=0.031416 \mathrm{~cm}^{2}$

$\mathrm{M}_{1}=\quad 0.03018$

$\mathrm{M}_{2}=\quad 1.04095$

$\mathrm{C}=\mathrm{M}_{1} \mathrm{~S} /\left(\mathrm{G}_{\mathrm{Hg}}-1\right)=0.0005418$ for $15^{\circ}$ to $25^{\circ}$

Remarks 


\section{amec $^{\theta}$}

\section{HYDRAULIC CONDUCTIVITY}

\begin{tabular}{|c|c|c|c|}
\hline Project No. & $6155-08-0031.34$ & Tested By & $J W \& J L$ \\
\hline Project Name & SDU-6 Task 3 Grout Samples & Test Date & $6 / 15 / 2012$ \\
\hline Boring No. & V6.0-2 & Reviewed By & $J W \partial \gamma$ \\
\hline Sample No. & V6.0-2 & Review Date & $8 / 1 / 2012$ \\
\hline Sample Depth & $N / A$ & Lab No. & 11606 \\
\hline
\end{tabular}

Sample Description Grout Core

\begin{tabular}{|l|l|}
\multicolumn{1}{c}{ ASTM D5084 - Method F (CVFH) } \\
\hline Sample Type: & Core \\
\hline Sample Orientation: & Vertical \\
\hline Initial Water Content, \%: & 43.4 \\
\hline Wet Unit Weight, pcf: & 107.2 \\
\hline Dry Unit Weight, pcf: & 74.8 \\
\hline Compaction, \%: & N/A \\
\hline Hydraulic Conductivity, cm/sec. @20 ${ }^{\circ} \mathrm{C}$ & $1.6 E-09$ \\
\hline
\end{tabular}

Remarks: 
SRNL-STI-2012-00522

Revision 0

\section{Equipment List}

SRNS Delivery Order No. 34

Subcontract No. AC54317N

\begin{tabular}{|c|c|}
\hline Equipment Name & Laboratory ID \\
\hline Oven & 109 \\
\hline Balance & 416 \\
\hline Thermometer & 2866 \\
\hline Caliper & 2373 \\
\hline Pressure Transducers & 3638 \\
\hline & \\
\hline & \\
\hline & \\
\hline & \\
\hline & \\
\hline & \\
\hline
\end{tabular}


SRNL-STI-2012-00522

Revision 0

PERMEABILITY TEST

(ASTM D5084 - 03) (Method F, Constant Volume Falling Head)

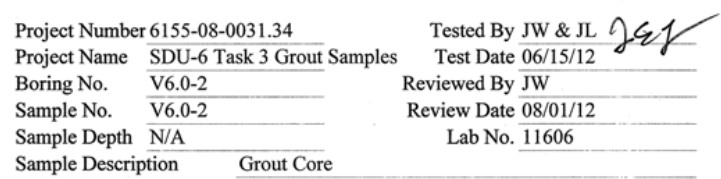

Sample Description Grout Core

\begin{tabular}{|c|r|c|r|c|r|r|}
\hline \multicolumn{4}{|c|}{ Initial Sample Data } & \multicolumn{2}{c|}{ Final Sample Data } \\
\hline \multicolumn{2}{|c|}{ Length, in } & \multicolumn{2}{|c|}{ Diameter, in } & Pan No. & \multicolumn{1}{|c|}{ N/A } \\
\hline Location 1 & 4.508 & Location 1 & 3.009 & Wet Soil+Pan, grams & 902.40 \\
\hline Location 2 & 4.531 & Location 2 & 3.005 & Dry Soil + Pan, grams & 629.27 \\
\hline Location3 & 4.519 & Location 3 & 3.001 & Pan Weight, grams & 0 \\
\hline Average & 4.519 & Average & 3.005 & Moisture Content, \% & 43.4 \\
\hline Volume, in & 3 & 32.05 & Wet Soil + Tare, grams & 902.16 & Dry Unit Weight, pcf & 74.8 \\
\hline SG Assumed & 2.40 & Tare Weight, grams & 0.00 & Saturation, \% & 103.9 \\
\hline Soil Sample Wt., g & 902.16 & Dry Soil + Tare, grams & 629.27 & Diameter, in. & N/A \\
\hline Dry UW, pcf & 74.8 & Moisture Content, \% & 43.4 & Length, in. & N/A \\
\hline Saturation, \% & 103.8 & & & Volume, in & N/A \\
\hline
\end{tabular}

\section{$\operatorname{amec}^{\theta}$}

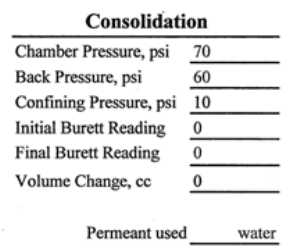

\begin{tabular}{|r|c|c|c|c|c|c|c|c|c|}
\hline $\begin{array}{c}\text { Elapsed Time } \\
(\mathrm{sec})\end{array}$ & $\begin{array}{c}\mathrm{z}_{\mathrm{o}} \\
(\mathrm{cm})\end{array}$ & $\begin{array}{c}\mathrm{za} \\
(\mathrm{cm})\end{array}$ & $\begin{array}{c}\mathrm{zb} \\
(\mathrm{cm})\end{array}$ & $\begin{array}{c}\Delta \mathrm{z}_{\mathrm{p}} \\
(\mathrm{cm})\end{array}$ & $\begin{array}{c}\text { Temp } \\
\left({ }^{\circ} \mathrm{C}\right)\end{array}$ & $\begin{array}{c}\text { Intial } \\
\text { Hydraulic } \\
\text { Gradient }\end{array}$ & $\begin{array}{c}\text { Final } \\
\text { Hydraulic } \\
\text { Gradient }\end{array}$ & $\begin{array}{c}\mathrm{k} \\
\mathrm{cm} / \mathrm{sec}\end{array}$ & $\begin{array}{c}\mathrm{k} \\
\mathrm{cm} / \mathrm{sec} \\
\mathrm{at} 20^{\circ} \mathrm{C}\end{array}$ \\
\hline 3120 & 1.70 & 24.40 & 24.20 & 0.20 & 22.1 & 24.9 & 24.6 & $1.78 \mathrm{E}-09$ & $1.69 \mathrm{E}-09$ \\
\hline 6600 & 1.70 & 24.40 & 24.10 & 0.30 & 22.1 & 24.9 & 24.5 & $1.26 \mathrm{E}-09$ & $1.20 \mathrm{E}-09$ \\
\hline 10980 & 1.50 & 26.40 & 25.30 & 1.10 & 22.3 & 27.3 & 26.0 & $2.58 \mathrm{E}-09$ & $2.44 \mathrm{E}-09$ \\
\hline 13748 & 1.50 & 26.40 & 25.10 & 1.30 & 22.4 & 27.3 & 25.8 & $2.45 \mathrm{E}-09$ & $2.31 \mathrm{E}-09$ \\
\hline 68880 & 1.50 & 26.40 & 23.00 & 3.40 & 22.3 & 27.3 & 23.4 & $1.34 \mathrm{E}-09$ & $1.27 \mathrm{E}-09$ \\
\hline 78360 & 1.50 & 26.40 & 22.80 & 3.60 & 22.3 & 27.3 & 23.2 & $1.25 \mathrm{E}-09$ & $1.19 \mathrm{E}-09$ \\
\hline 90120 & 1.50 & 26.40 & 22.00 & 4.40 & 22.6 & 27.3 & 22.3 & $1.36 \mathrm{E}-09$ & $1.28 \mathrm{E}-09$ \\
\hline
\end{tabular}

\begin{tabular}{|c|c|c|c|c|}
\hline No. of Trials & $\begin{array}{c}\text { Sample } \\
\text { Type }\end{array}$ & $\begin{array}{c}\text { Max. Density } \\
(\mathrm{pcf})\end{array}$ & $\begin{array}{c}\text { Compaction } \\
\%\end{array}$ & $\begin{array}{c}\text { Sample } \\
\text { Orientation }\end{array}$ \\
\hline 7 & Core & N/A & N/A & Vertical \\
\hline
\end{tabular}

Avg. $\mathrm{k}$ at $20^{\circ} \mathrm{C} \quad 1.6 \mathrm{E}-09 \mathrm{~cm} / \mathrm{sec}$

$$
\begin{aligned}
& \mathrm{a}_{\mathrm{a}}=0.76712 \mathrm{~cm}^{2} \\
& \mathrm{~A}=\frac{45.76 \mathrm{~cm}^{2}}{\mathrm{~L}}=\frac{11.48 \mathrm{~cm}}{}
\end{aligned}
$$

$\mathrm{S}=\mathrm{L} / \mathrm{A}=0.25088 \mathrm{1} / \mathrm{cm}$

$a_{\mathrm{p}}=0.031416 \mathrm{~cm}^{2}$

$\mathrm{M}_{1}=0.03018$

$\mathrm{M}_{2}=\quad 1.04095$

$\mathrm{C}=\mathrm{M}_{1} \mathrm{~S} /\left(\mathrm{G}_{\mathrm{Hg}}-1\right)=0.0006023$ for $15^{\circ}$ to $25^{\circ}$

Remarks:

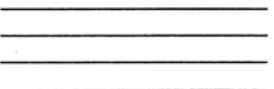

RCN: SRS256

Page 6 of 46 


\section{amec $^{\theta}$}

\section{HYDRAULIC CONDUCTIVITY}

$\begin{array}{llll}\text { Project No. } & \mathbf{6 1 5 5 - 0 8 - 0 0 3 1 . 3 4} & \text { Tested By } & \boldsymbol{J W} \& \boldsymbol{J L} \\ \text { Project Name } & \text { SDU-6 Task 3 Grout Samples } & \text { Test Date } & 6 / 15 / 2012 \\ \text { Boring No. } & \text { V6.0-3 } & \text { Reviewed By } & \boldsymbol{J} W \mathcal{C} \mathcal{J} \\ \text { Sample No. } & \mathbf{V 6 . 0 - 3} & \text { Review Date } & 8 / 1 / 2012 \\ \text { Sample Depth } & \text { N/A } & \text { Lab No. } & 11607\end{array}$

Sample Description Grout Core

ASTM D5084 - Method F (CVFH)

\begin{tabular}{|l|l|}
\hline Sample Type: & Core \\
\hline Sample Orientation: & Vertical \\
\hline Initial Water Content, \%: & 44.0 \\
\hline Wet Unit Weight, pcf: & 107.0 \\
\hline Dry Unit Weight, pcf: & 74.3 \\
\hline Compaction, \%: & N/A \\
\hline Hydraulic Conductivity, cm/sec. @20 C & $\mathbf{2 . 6 E - 1 0}$ \\
\hline
\end{tabular}

Remarks: 
SRNL-STI-2012-00522

Revision 0

PERMEABILITY TEST

(ASTM D5084 - 03) (Method F, Constant Volume Falling Head)

ameco

Project Number 6155-08-0031.34

Tested By JW \& JL

Project Name SDU-6 Task 3 Grout Samples Test Date 06/15/12

Boring No. V6.0-3 Reviewed By JW OCC

Sample No. V6.0-3 Review Date 08/01/12

Sample Depth N/A Lab No. 11607

Sample Description Grout Core

\begin{tabular}{|c|c|c|c|c|c|}
\hline \multicolumn{4}{|c|}{ Initial Sample Data } & \multicolumn{2}{|c|}{ Final Sample Data } \\
\hline Length, i & & Diameter, in & & Pan No. & $\mathrm{N} / \mathrm{A}$ \\
\hline Location 1 & 4.274 & Location 1 & 3.012 & Wet Soil+Pan, grams & 856.37 \\
\hline Location 2 & 4.291 & Location 2 & 2.999 & Dry Soil + Pan, grams & 594.60 \\
\hline Location 3 & 4.349 & Location 3 & 2.998 & Pan Weight, grams & \\
\hline Average & 4.305 & Average & 3.003 & Moisture Content, \% & 44.0 \\
\hline Volume, in ${ }^{3}$ & 30.49 & Wet Soil + Tare, grams & 855.96 & Dry Unit Weight, pef & 74.3 \\
\hline SG Assumed & 2.40 & Tare Weight, grams & 0.00 & Saturation, \% & 104.0 \\
\hline Soil Sample Wt., g & 855.96 & Dry Soil +Tare, grams & 594.60 & Diameter, in. & N/A \\
\hline Dry UW, pef & 74.3 & Moisture Content, \% & 44.0 & Length, in. & N/A \\
\hline Saturation, $\%$ & 103.9 & & & Volume, in ${ }^{3}$ & N/A \\
\hline
\end{tabular}

Consolidation

Chamber Pressure, psi 70

Back Pressure, psi

Confining Pressure, psi 10

Initial Burett Reading

Final Burett Reading

Final Burett Reading

Volume Change, $\mathrm{cc}$

Permeant used water

\begin{tabular}{|r|r|r|r|r|r|r|r|r|r|}
\hline $\begin{array}{c}\text { Elapsed Time } \\
(\mathrm{sec})\end{array}$ & $\begin{array}{c}\mathrm{z}_{\mathrm{o}} \\
(\mathrm{cm})\end{array}$ & $\begin{array}{c}\mathrm{za} \\
(\mathrm{cm})\end{array}$ & $\begin{array}{c}\mathrm{zb} \\
(\mathrm{cm})\end{array}$ & $\begin{array}{c}\Delta \mathrm{z}_{\mathrm{p}} \\
(\mathrm{cm})\end{array}$ & $\begin{array}{c}\text { Temp } \\
\left({ }^{\circ} \mathrm{C}\right)\end{array}$ & $\begin{array}{c}\text { Intial } \\
\text { Hydraulic }\end{array}$ & $\begin{array}{c}\text { Final } \\
\text { Hydraulic } \\
\text { Gradient }\end{array}$ & $\begin{array}{c}\mathrm{k} \\
\mathrm{cm} / \mathrm{sec}\end{array}$ & \multicolumn{1}{c|}{$\begin{array}{c}\mathrm{k} \\
\mathrm{cm} / \mathrm{sec} \\
\mathrm{at} 20^{\circ} \mathrm{C}\end{array}$} \\
\hline 78480 & 1.70 & 25.40 & 24.20 & 1.20 & 22.1 & 27.2 & 25.8 & $3.96 \mathrm{E}-10$ & $3.77 \mathrm{E}-10$ \\
\hline 7380 & 1.70 & 24.50 & 24.40 & 0.10 & 22.1 & 26.2 & 26.1 & $3.56 \mathrm{E}-10$ & $3.39 \mathrm{E}-10$ \\
\hline 14990 & 1.70 & 24.50 & 24.30 & 0.20 & 23.6 & 26.2 & 26.0 & $3.52 \mathrm{E}-10$ & $3.23 \mathrm{E}-10$ \\
\hline 68280 & 1.70 & 24.50 & 23.90 & 0.60 & 23.6 & 26.2 & 25.5 & $2.34 \mathrm{E}-10$ & $2.15 \mathrm{E}-10$ \\
\hline 80100 & 1.70 & 24.50 & 23.80 & 0.70 & 22.3 & 26.2 & 25.4 & $2.33 \mathrm{E}-10$ & $2.21 \mathrm{E}-10$ \\
\hline 96120 & 1.70 & 24.50 & 23.70 & 0.80 & 22.4 & 26.2 & 25.3 & $2.22 \mathrm{E}-10$ & $2.10 \mathrm{E}-10$ \\
\hline 154500 & 1.70 & 24.50 & 23.50 & 1.00 & 22.4 & 26.2 & 25.0 & $1.74 \mathrm{E}-10$ & $1.64 \mathrm{E}-10$ \\
\hline
\end{tabular}

\begin{tabular}{|c|c|c|c|c|}
\hline No. of Trials & $\begin{array}{c}\text { Sample } \\
\text { Type }\end{array}$ & $\begin{array}{c}\text { Max. Density } \\
(\mathrm{pcf})\end{array}$ & $\begin{array}{c}\text { Compaction } \\
\%\end{array}$ & $\begin{array}{c}\text { Sample } \\
\text { Orientation }\end{array}$ \\
\hline 7 & Core & N/A & N/A & Vertical \\
\hline
\end{tabular}

Avg. $\mathrm{k}$ at $20^{\circ} \mathrm{C} \quad 2.6 \mathrm{E}-10 \mathrm{~cm} / \mathrm{sec}$

$\begin{array}{rr}\mathrm{a}_{\mathrm{s}}= & 0.76712 \mathrm{~cm}^{2} \\ \mathrm{~A} & =45.69 \mathrm{~cm}^{2} \\ \mathrm{~L} & =\quad 10.93 \mathrm{~cm} \\ \mathrm{~S}=\mathrm{L} / \mathrm{A} & =0.23928 \mathrm{l} / \mathrm{cm}\end{array}$

$a_{p}=0.031416 \mathrm{~cm}^{2}$

$\mathrm{M}_{1}=0.03018$

$\mathrm{M}_{2}=1.04095$

$\mathrm{S}=\mathrm{L} / \mathrm{A}=0.23928 \mathrm{1} / \mathrm{cm}$

$\mathrm{C}=\mathrm{M}_{1} \mathrm{~S} /\left(\mathrm{G}_{\mathrm{H}}-1\right)=0.0005745$ for $15^{\circ}$ to $25^{\circ}$

Remarks

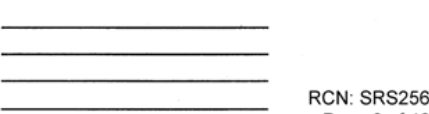

列 256 


\section{$\operatorname{amec}^{\theta}$}

\section{HYDRAULIC CONDUCTIVITY}

\begin{tabular}{|c|c|c|c|}
\hline Project No. & $6155-08-0031.34$ & Tested By & $J W \& J L$ \\
\hline Project Name & SDU-6 Task 3 Grout Samples & Test Date & $6 / 15 / 2012$ \\
\hline Boring No. & V3.0-1 & Reviewed By & $J W \partial \varepsilon \gamma$ \\
\hline Sample No. & V3.0-1 & Review Date & $8 / 1 / 2012$ \\
\hline Sample Depth & $N / A$ & Lab No. & 11608 \\
\hline
\end{tabular}

Sample Description Grout Core

ASTM D5084 - Method F (CVFH)

\begin{tabular}{|l|l|}
\hline Sample Type: & Core \\
\hline Sample Orientation: & Vertical \\
\hline Initial Water Content, \%: & 44.8 \\
\hline Wet Unit Weight, pcf: & 107.1 \\
\hline Dry Unit Weight, pcf: & 74.0 \\
\hline Compaction, \%: & N/A \\
\hline Hydraulic Conductivity, cm/sec. @20 ${ }^{\circ} \mathrm{C}$ & $\mathbf{2 . 3 E - 0 9}$ \\
\hline
\end{tabular}

Remarks: 
SRNL-STI-2012-00522

Revision 0

PERMEABILITY TEST

(ASTM D5084 - 03) (Method F, Constant Volume Falling Head)

\section{$\operatorname{amec}^{\theta}$}

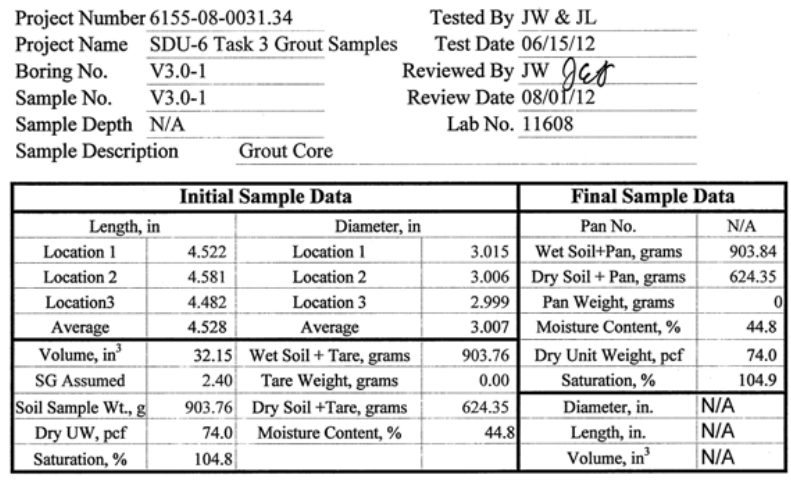

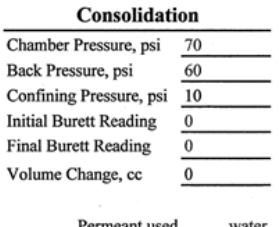

\begin{tabular}{|r|c|c|c|r|r|r|r|r|r|}
\hline $\begin{array}{c}\text { Elapsed Time } \\
(\mathrm{sec})\end{array}$ & $\begin{array}{c}\mathrm{z}_{\mathrm{o}} \\
(\mathrm{cm})\end{array}$ & $\begin{array}{c}\mathrm{za} \\
(\mathrm{cm})\end{array}$ & $\begin{array}{c}\mathrm{zb} \\
(\mathrm{cm})\end{array}$ & $\begin{array}{c}\Delta \mathrm{z}_{\mathrm{p}} \\
(\mathrm{cm})\end{array}$ & $\begin{array}{c}\text { Temp } \\
\left({ }^{\circ} \mathrm{C}\right)\end{array}$ & $\begin{array}{c}\text { Intial } \\
\text { Hydraulic } \\
\text { Gradient }\end{array}$ & $\begin{array}{c}\text { Final } \\
\text { Hydraulic } \\
\text { Gradient }\end{array}$ & $\begin{array}{c}\mathrm{k} \\
\mathrm{cm} / \mathrm{sec}\end{array}$ & $\begin{array}{c}\mathrm{k} \\
\mathrm{cm} / \mathrm{sec} \\
\mathrm{at} 20^{\circ} \mathrm{C}\end{array}$ \\
\hline 4860 & 1.50 & 22.80 & 22.30 & 0.50 & 22.1 & 23.3 & 22.7 & $3.07 \mathrm{E}-09$ & $2.92 \mathrm{E}-09$ \\
\hline 8100 & 1.50 & 22.80 & 22.00 & 0.80 & 22.1 & 23.3 & 22.4 & $2.97 \mathrm{E}-09$ & $2.82 \mathrm{E}-09$ \\
\hline 12000 & 1.50 & 22.80 & 21.80 & 1.00 & 22.1 & 23.3 & 22.1 & $2.52 \mathrm{E}-09$ & $2.39 \mathrm{E}-09$ \\
\hline 22430 & 1.50 & 22.80 & 21.00 & 1.80 & 22.1 & 23.3 & 21.2 & $2.47 \mathrm{E}-09$ & $2.35 \mathrm{E}-09$ \\
\hline 79080 & 1.50 & 22.80 & 18.20 & 4.60 & 22.1 & 23.3 & 18.0 & $1.94 \mathrm{E}-09$ & $1.85 \mathrm{E}-09$ \\
\hline 85080 & 1.50 & 22.80 & 18.00 & 4.80 & 22.1 & 23.3 & 17.8 & $1.89 \mathrm{E}-09$ & $1.80 \mathrm{E}-09$ \\
\hline 92880 & 1.50 & 22.80 & 17.80 & 5.00 & 22.1 & 23.3 & 17.6 & $1.82 \mathrm{E}-09$ & $1.73 \mathrm{E}-09$ \\
\hline
\end{tabular}

\begin{tabular}{|c|c|c|c|c|}
\hline No. of Trials & Sample & Max. Density & Compaction & Sample \\
\hline & Type & (pef) & $\%$ & Orientation \\
\hline 7 & Core & N/A & N/A & Vertical \\
\hline
\end{tabular}

Avg. k at $20^{\circ} \mathrm{C} \quad 2.3 \mathrm{E}-09 \mathrm{~cm} / \mathrm{sec}$

$$
\begin{aligned}
& \mathrm{a}_{\mathrm{a}}=0.76712 \mathrm{~cm}^{2} \\
& \mathrm{~A}=-45.81 \mathrm{~cm}^{2} \\
& \mathrm{~L}=-11.50 \mathrm{~cm}
\end{aligned}
$$

$\mathrm{S}=\mathrm{L} / \mathrm{A}=0.25110 \mathrm{l} / \mathrm{cm}$

$\begin{aligned} \mathrm{a}_{\mathrm{p}}= & 0.031416 \mathrm{~cm}^{2} \\ \mathrm{M}_{1}= & 0.03018 \\ \mathrm{M}_{2}= & 1.04095 \\ \mathrm{C}=\mathrm{M}_{1} \mathrm{~S} /\left(\mathrm{G}_{\mathrm{Hz}}-1\right)= & 0.0006029 \text { for } 15^{\circ} \text { to } 25^{\circ}\end{aligned}$

Remarks:

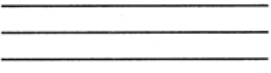

RCN: SRS256 Page 10 of 46 


\section{amec $^{\theta}$}

\section{HYDRAULIC CONDUCTIVITY}

$\begin{array}{llll}\text { Project No. } & \text { 6155-08-0031.34 } & \text { Tested By } & \text { JEL } \\ \text { Project Name } & \text { SDU-6 Task 3 Grout Samples } & \text { Test Date } & 6 / 27 / 2012 \\ \text { Boring No. } & \text { V3.0-2 } & \text { Reviewed By } & \text { JW } \text { G4f } \\ \text { Sample No. } & \text { V3.0-2 } & \text { Review Date } & 8 / 1 / 2012 \\ \text { Sample Depth } & \text { N/A } & \text { Lab No. } & 11609\end{array}$

Sample Description Grout Core

ASTM D5084 - Method F (CVFH)

\begin{tabular}{|l|l|}
\hline Sample Type: & Core \\
\hline Sample Orientation: & Vertical \\
\hline Initial Water Content, \%: & 44.5 \\
\hline Wet Unit Weight, pcf: & 107.5 \\
\hline Dry Unit Weight, pcf: & 74.4 \\
\hline Compaction, \%: & N/A \\
\hline Hydraulic Conductivity, cm/sec. @20 ${ }^{\circ} \mathrm{C}$ & $\mathbf{2 . 0 E - 0 9}$ \\
\hline
\end{tabular}

Remarks: 
SRNL-STI-2012-00522

Revision 0

PERMEABILITY TEST

(ASTM D5084 - 03) (Method F, Constant Volume Falling Head)

Project Number 6155-08-0031.34

Project Name SDU-6 Task 3 Grout Samples Test Date 06/27/12

Boring No. V3.0-2 Reviewed By JW $\mathrm{g}_{\varepsilon f}$

Sample No. V3.0-2 Review Date 08/01/12

Sample Depth N/A Lab No. 11609

Sample Description Grout Core

\begin{tabular}{|c|r|c|r|c|r|}
\hline \multicolumn{4}{|c|}{ Initial Sample Data } & \multicolumn{2}{c|}{ Final Sample Data } \\
\hline Length, in & \multicolumn{2}{|c|}{ Diameter, in } & Pan No. & N/A \\
\hline Location 1 & 4.416 & Location 1 & 2.994 & Wet Soil+Pan, grams & 882.14 \\
\hline Location 2 & 4.408 & Location 2 & 3.009 & Dry Soil + Pan, grams & 610.50 \\
\hline Location3 & 4.452 & Location 3 & 2.992 & Pan Weight, grams & 0 \\
\hline Average & 4.425 & Average & 2.998 & Moisture Content, \% & 44.5 \\
\hline Volume, in & 31.25 & Wet Soil + Tare, grams & 882.05 & Dry Unit Weight, pef & 74.4 \\
\hline SG Assumed & 2.40 & Tare Weight, grams & 0.00 & Saturation, \% & 105.5 \\
\hline Soil Sample Wt., g & 882.05 & Dry Soil + Tare, grams & 610.50 & Diameter, in. & N/A \\
\hline Dry UW, pcf & 74.4 & Moisture Content, \% & 44.5 & Length, in. & N/A \\
\hline Saturation, \% & 105.5 & & & Volume, in ${ }^{3}$ & N/A \\
\hline
\end{tabular}

\begin{tabular}{|r|r|r|r|r|r|r|r|r|r|}
\hline $\begin{array}{c}\text { Elapsed Time } \\
(\mathrm{sec})\end{array}$ & $\begin{array}{c}\mathrm{z}_{\mathrm{o}} \\
(\mathrm{cm})\end{array}$ & $\begin{array}{c}\mathrm{za} \\
(\mathrm{cm})\end{array}$ & $\begin{array}{c}\mathrm{zb} \\
(\mathrm{cm})\end{array}$ & $\begin{array}{c}\Delta \mathrm{z}_{\mathrm{p}} \\
(\mathrm{cm})\end{array}$ & $\begin{array}{c}\text { Temp } \\
\left({ }^{\circ} \mathrm{C}\right)\end{array}$ & $\begin{array}{c}\text { Intial } \\
\text { Hydraulic } \\
\text { Gradient }\end{array}$ & $\begin{array}{c}\text { Final } \\
\text { Hydraulic } \\
\text { Gradient }\end{array}$ & $\begin{array}{c}\mathrm{k} \\
\mathrm{cm} / \mathrm{sec}\end{array}$ & $\begin{array}{c}\mathrm{k} \\
\mathrm{cm} / \mathrm{sec} \\
\mathrm{at} 20{ }^{\circ} \mathrm{C}\end{array}$ \\
\hline 5220 & 1.50 & 22.80 & 22.40 & 0.40 & 22.9 & 23.8 & 23.4 & $2.24 \mathrm{E}-09$ & $2.09 \mathrm{E}-09$ \\
\hline 7890 & 1.50 & 22.80 & 22.10 & 0.70 & 22.9 & 23.8 & 23.0 & $2.61 \mathrm{E}-09$ & $2.44 \mathrm{E}-09$ \\
\hline 15840 & 1.50 & 22.80 & 22.05 & 0.75 & 22.9 & 23.8 & 22.9 & $1.40 \mathrm{E}-09$ & $1.30 \mathrm{E}-09$ \\
\hline 3600 & 1.50 & 24.80 & 24.20 & 0.60 & 23.0 & 26.1 & 25.4 & $4.47 \mathrm{E}-09$ & $4.16 \mathrm{E}-09$ \\
\hline 14100 & 1.50 & 24.80 & 24.10 & 0.70 & 23.1 & 26.1 & 25.2 & $1.34 \mathrm{E}-09$ & $1.24 \mathrm{E}-09$ \\
\hline 3600 & 1.50 & 26.40 & 26.20 & 0.20 & 23.1 & 27.8 & 27.6 & $1.38 \mathrm{E}-09$ & $1.28 \mathrm{E}-09$ \\
\hline 6120 & 1.50 & 26.40 & 26.10 & 0.30 & 23.1 & 27.8 & 27.5 & $1.22 \mathrm{E}-09$ & $1.14 \mathrm{E}-09$ \\
\hline
\end{tabular}

\begin{tabular}{|c|c|c|c|c|}
\hline No. of Trials & $\begin{array}{c}\text { Sample } \\
\text { Type }\end{array}$ & $\begin{array}{c}\text { Max. Density } \\
(\mathrm{pcf})\end{array}$ & $\begin{array}{c}\text { Compaction } \\
\%\end{array}$ & $\begin{array}{c}\text { Sample } \\
\text { Orientation }\end{array}$ \\
\hline 7 & Core & N/A & N/A & Vertical \\
\hline
\end{tabular}

Avg. $\mathrm{k}$ at $20^{\circ} \mathrm{C} \quad 2.0 \mathrm{E}-09 \mathrm{~cm} / \mathrm{sec}$

$$
\begin{aligned}
\mathrm{a}_{2} & =0.76712 \mathrm{~cm}^{2} \\
\mathrm{~A} & =\frac{45.55 \mathrm{~cm}^{2}}{\mathrm{~L}}= \\
\mathrm{S}=\mathrm{L} / \mathrm{A} & =0.24675 \mathrm{~cm}
\end{aligned}
$$

$\mathrm{a}_{\mathrm{p}}=0.031416 \mathrm{~cm}^{2}$

$\mathrm{M}_{1}=0.03018$

$\mathrm{M}_{2}=1.04095$

$\mathrm{C}=\mathrm{M}_{1} \mathrm{~S} /\left(\mathrm{G}_{\mathrm{Hz}}-1\right)=0.0005924$ for $15^{\circ}$ to $25^{\circ}$

Remarks

\section{$\operatorname{amec}^{\theta}$}

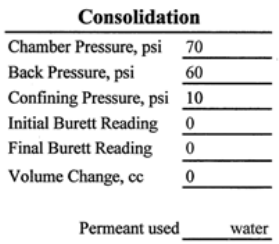

Permeant used 


\section{amec $^{\theta}$}

\section{HYDRAULIC CONDUCTIVITY}

$\begin{array}{llll}\text { Project No. } & \mathbf{6 1 5 5 - 0 8 - 0 0 3 1 . 3 4} & \text { Tested By } & \boldsymbol{J} \\ \text { Project Name } & \text { SDU-6 Task 3 Grout Samples } & \text { Test Date } & 6 / 27 / 2012 \\ \text { Boring No. } & \text { V3.0-3 } & \text { Reviewed By } & J W \text { VGO } \\ \text { Sample No. } & \text { V3.0-3 } & \text { Review Date } & 8 / 1 / 2012 \\ \text { Sample Depth } & \text { N/A } & \text { Lab No. } & 11610\end{array}$

Sample Description Grout Core

ASTM D5084 - Method F (CVFH)

\begin{tabular}{|l|l|}
\hline Sample Type: & Core \\
\hline Sample Orientation: & Vertical \\
\hline Initial Water Content, \%: & 44.4 \\
\hline Wet Unit Weight, pcf: & 108.7 \\
\hline Dry Unit Weight, pcf: & 75.3 \\
\hline Compaction, \%: & N/A \\
\hline Hydraulic Conductivity, cm/sec. @20 ${ }^{\circ} \mathrm{C}$ & $1.4 E-09$ \\
\hline
\end{tabular}

Remarks: 
SRNL-STI-2012-00522

Revision 0

PERMEABILITY TEST

(ASTM D5084 - 03) (Method F, Constant Volume Falling Head)

ameco

Project Number 6155-08-0031.34

Tested By JL

Project Name SDU-6 Task 3 Grout Samples Test Date 06/27/12

Boring No. V3.0-3 Reviewed By JW O\&Y

Sample No. V3.0-3 Review Date 08/01/12

Sample Depth N/A Lab No. 11610

Sample Description Grout Core

\begin{tabular}{|c|c|c|c|c|c|}
\hline \multicolumn{4}{|c|}{ Initial Sample Data } & \multicolumn{2}{|c|}{ Final Sample Data } \\
\hline Length, i & & Diameter, in & & Pan No. & $\mathrm{N} / \mathrm{A}$ \\
\hline Location 1 & 4.397 & Location 1 & 2.911 & Wet Soil+Pan, grams & 870.30 \\
\hline Location 2 & 4.401 & Location 2 & 3.005 & Dry Soil + Pan, grams & 602.76 \\
\hline Location 3 & 4.397 & Location 3 & 2.998 & Pan Weight, grams & \\
\hline Average & 4.398 & Average & 2.971 & Moisture Content, \% & 44.4 \\
\hline Volume, in ${ }^{3}$ & 30.50 & Wet Soil + Tare, grams & 870.47 & Dry Unit Weight, pef & 75.3 \\
\hline SG Assumed & 2.40 & Tare Weight, grams & 0.00 & Saturation, \% & 107.7 \\
\hline Soil Sample Wt., g & 870.47 & Dry Soil +Tare, grams & 602.76 & Diameter, in. & N/A \\
\hline Dry UW, pef & 75.3 & Moisture Content, \% & 44.4 & Length, in. & N/A \\
\hline Saturation, $\%$ & 107.8 & & & Volume, in ${ }^{3}$ & N/A \\
\hline
\end{tabular}

Consolidation Chamber Pressure, psi 70 Back Pressure, psi

Confining Pressure, psi 1

Initial Burett Reading

Final Burett Reading

Final Burett Reading

Volume Change, cc

Permeant used

\begin{tabular}{l}
$\frac{70}{60}$ \\
\hline 10 \\
\hline 0 \\
\hline 0 \\
\hline 0 \\
\hline
\end{tabular}

\begin{tabular}{|r|r|r|r|r|r|r|r|r|r|}
\hline $\begin{array}{c}\text { Elapsed Time } \\
(\mathrm{sec})\end{array}$ & $\begin{array}{c}\mathrm{z}_{\mathrm{o}} \\
(\mathrm{cm})\end{array}$ & $\begin{array}{c}\mathrm{za} \\
(\mathrm{cm})\end{array}$ & $\begin{array}{c}\mathrm{zb} \\
(\mathrm{cm})\end{array}$ & $\begin{array}{c}\Delta \mathrm{z}_{\mathrm{p}} \\
(\mathrm{cm})\end{array}$ & $\begin{array}{c}\text { Temp } \\
\left({ }^{\circ} \mathrm{C}\right)\end{array}$ & $\begin{array}{c}\text { Intial } \\
\text { Hydraulic } \\
\text { Gradient }\end{array}$ & $\begin{array}{c}\text { Final } \\
\text { Hydraulic } \\
\text { Gradient }\end{array}$ & $\begin{array}{c}\mathrm{k} \\
\mathrm{cm} / \mathrm{sec}\end{array}$ & $\begin{array}{c}\mathrm{k} \\
\mathrm{cm} / \mathrm{sec} \\
\mathrm{at} 20^{\circ} \mathrm{C}\end{array}$ \\
\hline 4680 & 1.70 & 23.50 & 23.20 & 0.30 & 23.1 & 24.5 & 24.2 & $1.85 \mathrm{E}-09$ & $1.72 \mathrm{E}-09$ \\
\hline 10200 & 1.70 & 23.50 & 22.90 & 0.60 & 22.9 & 24.5 & 23.8 & $1.71 \mathrm{E}-09$ & $1.59 \mathrm{E}-09$ \\
\hline 14880 & 1.70 & 23.50 & 22.80 & 0.70 & 22.9 & 24.5 & 23.7 & $1.37 \mathrm{E}-09$ & $1.28 \mathrm{E}-09$ \\
\hline 19260 & 1.70 & 23.50 & 22.60 & 0.90 & 22.9 & 24.5 & 23.5 & $1.37 \mathrm{E}-09$ & $1.28 \mathrm{E}-09$ \\
\hline 21300 & 1.70 & 23.50 & 22.50 & 1.00 & 22.9 & 24.5 & 23.4 & $1.38 \mathrm{E}-09$ & $1.29 \mathrm{E}-09$ \\
\hline 23340 & 1.70 & 23.50 & 22.40 & 1.10 & 22.9 & 24.5 & 23.2 & $1.39 \mathrm{E}-09$ & $1.29 \mathrm{E}-09$ \\
\hline 25200 & 1.70 & 23.50 & 22.30 & 1.20 & 22.9 & 24.5 & 23.1 & $1.40 \mathrm{E}-09$ & $1.31 \mathrm{E}-09$ \\
\hline
\end{tabular}

\begin{aligned} No. of Trials & $\begin{array}{c}\text { Samp } \\ \text { Typp }\end{array} \\$\hline 7 & Core \\ $\mathrm{a}_{\mathrm{a}}= & 0.76712 \mathrm{~cm}^{2} \\ \mathrm{~A}= & 44.74 \mathrm{~cm}^{2} \\ \mathrm{~L}= & 11.17 \mathrm{~cm} \\ \mathrm{~S}=\mathrm{L} / \mathrm{A}= & 0.24972 \mathrm{l} / \mathrm{cm}\end{aligned}$

Sample Max. Density Compaction Sample

\begin{tabular}{c|c|c|c|}
\hline Type & $(\mathrm{pcf})$ & $\%$ & Orientation \\
\hline Core & N/A & N/A & Vertical \\
\hline
\end{tabular}

Avg. $\mathrm{k}$ at $20^{\circ} \mathrm{C} \quad 1.4 \mathrm{E}-09 \mathrm{~cm} / \mathrm{sec}$

$\begin{aligned} \mathrm{a}_{p}= & 0.031416 \mathrm{~cm}^{2} \\ \mathrm{M}_{1}= & 0.03018 \\ \mathrm{M}_{2}= & 1.04095 \\ \mathrm{C}=\mathrm{M}_{1} \mathrm{~S} /\left(\mathrm{G}_{\mathrm{Hg}}-1\right)= & 0.0005996 \text { for } 15^{\circ} \text { to } 25^{\circ}\end{aligned}$

Remarks

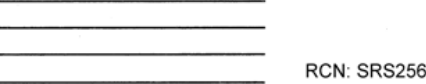




\section{amec $^{\circ}$}

\section{HYDRAULIC CONDUCTIVITY}

\begin{tabular}{|c|c|c|c|}
\hline Project No. & $6155-08-0031.34$ & Tested By & $J E L$ \\
\hline Project Name & SDU-6 Task 3 Grout Samples & Test Date & $6 / 28 / 2012$ \\
\hline Boring No. & $H 1.5-1$ & Reviewed By & JW \\
\hline Sample No. & H1.5-1 & Review Date & $8 / 1 / 2012$ \\
\hline Sample Depth & $N / A$ & Lab No. & 11611 \\
\hline
\end{tabular}

Sample Description Grout Core

ASTM D5084 - Method F (CVFH)

\begin{tabular}{|l|l|}
\hline Sample Type: & Core \\
\hline Sample Orientation: & Vertical \\
\hline Initial Water Content, \%: & 44.3 \\
\hline Wet Unit Weight, pcf: & 105.8 \\
\hline Dry Unit Weight, pcf: & 73.3 \\
\hline Compaction, \%: & N/A \\
\hline Hydraulic Conductivity, cm/sec. @20 ${ }^{\circ} \mathrm{C}$ & $\mathbf{4 . 0 E - 0 8}$ \\
\hline
\end{tabular}

Remarks: 
SRNL-STI-2012-00522

Revision 0

PERMEABILITY TEST

(ASTM D5084 - 03) (Method F, Constant Volume Falling Head)

amec $^{\theta}$

Project Number 6155-08-0031.34

Tested By JEL

Project Name SDU-6 Task 3 Grout Samples Test Date 06/28/12

Boring No. H1.5-1 Reviewed By JW

Sample No. H1.5-1 Review Date 08/01/12

Sample Depth N/A Lab No. 1161

Sample Description Grout Core

\begin{tabular}{|c|c|c|c|c|c|}
\hline \multicolumn{4}{|c|}{ Initial Sample Data } & \multicolumn{2}{|c|}{ Final Sample Data } \\
\hline Length, i & & Diameter, in & & Pan No. & $\mathrm{N} / \mathrm{A}$ \\
\hline Location 1 & 4.657 & Location 1 & 3.014 & Wet Soil+Pan, grams & 922.99 \\
\hline Location 2 & 4.646 & Location 2 & 3.001 & Dry Soil + Pan, grams & 639.43 \\
\hline Location3 & 4.640 & Location 3 & 3.035 & Pan Weight, grams & \\
\hline Average & 4.648 & Average & 3.017 & Moisture Content, \% & 44.3 \\
\hline Volume, in ${ }^{3}$ & 33.22 & Wet Soil + Tare, grams & 922.89 & Dry Unit Weight, pef & 73.3 \\
\hline SG Assumed & 2.40 & Tare Weight, grams & 0.00 & Saturation, \% & 102.1 \\
\hline Soil Sample Wt., g & 922.89 & Dry Soil +Tare, grams & 639.43 & Diameter, in. & N/A \\
\hline Dry UW, pef & 73.3 & Moisture Content, \% & 44.3 & Length, in. & N/A \\
\hline Saturation, \% & 102.1 & & & Volume, in ${ }^{3}$ & N/A \\
\hline
\end{tabular}

\begin{tabular}{|c|c|}
\hline \multicolumn{2}{|c|}{ Consolidation } \\
\hline Chamber Pressure, psi & 70 \\
\hline Back Pressure, psi & 60 \\
\hline Confining Pressure, psi & 10 \\
\hline Initial Burett Reading & 0 \\
\hline Final Burett Reading & 0 \\
\hline Volume Change, cc & 0 \\
\hline
\end{tabular}

\begin{tabular}{|r|r|r|r|r|r|r|r|r|r|}
\hline $\begin{array}{c}\text { Elapsed Time } \\
(\mathrm{sec})\end{array}$ & $\begin{array}{c}\mathrm{z}_{\mathrm{o}} \\
(\mathrm{cm})\end{array}$ & $\begin{array}{c}\mathrm{za} \\
(\mathrm{cm})\end{array}$ & $\begin{array}{c}\mathrm{zb} \\
(\mathrm{cm})\end{array}$ & $\begin{array}{c}\Delta \mathrm{z}_{\mathrm{p}} \\
(\mathrm{cm})\end{array}$ & $\begin{array}{c}\text { Temp } \\
\left({ }^{\circ} \mathrm{C}\right)\end{array}$ & $\begin{array}{c}\text { Intial } \\
\text { Hydraulic }\end{array}$ & $\begin{array}{c}\text { Final } \\
\text { Hydraulic } \\
\text { Gradient }\end{array}$ & $\begin{array}{c}\mathrm{k} \\
\mathrm{Gm} / \mathrm{sec}\end{array}$ & $\begin{array}{c}\mathrm{k} \\
\mathrm{cm} / \mathrm{sec}\end{array}$ \\
\hline 2340 & 1.70 & 25.00 & 20.50 & 4.50 & 22.9 & 24.8 & 19.8 & $5.90 \mathrm{E}-08$ & $5.50 \mathrm{E}-08$ \\
\hline 4200 & 1.70 & 25.00 & 18.50 & 6.50 & 23.0 & 24.8 & 17.6 & $5.02 \mathrm{E}-08$ & $4.67 \mathrm{E}-08$ \\
\hline 9600 & 1.70 & 25.00 & 13.70 & 11.30 & 23.1 & 24.8 & 12.3 & $4.50 \mathrm{E}-08$ & $4.18 \mathrm{E}-08$ \\
\hline 4380 & 1.70 & 25.50 & 20.30 & 5.20 & 23.1 & 25.3 & 19.6 & $3.62 \mathrm{E}-08$ & $3.36 \mathrm{E}-08$ \\
\hline 4140 & 1.70 & 26.40 & 21.40 & 5.00 & 23.1 & 26.3 & 20.8 & $3.51 \mathrm{E}-08$ & $3.26 \mathrm{E}-08$ \\
\hline 6900 & 1.70 & 26.40 & 18.90 & 7.50 & 23.1 & 26.3 & 18.0 & $3.38 \mathrm{E}-08$ & $3.14 \mathrm{E}-08$ \\
\hline 1740 & 1.70 & 24.70 & 22.30 & 2.40 & 23.1 & 24.5 & 21.8 & $4.06 \mathrm{E}-08$ & $3.77 \mathrm{E}-08$ \\
\hline
\end{tabular}

\begin{tabular}{|c|c|c|c|c|}
\hline No. of Trials & $\begin{array}{c}\text { Sample } \\
\text { Type }\end{array}$ & $\begin{array}{c}\text { Max. Density Compaction } \\
(\mathrm{pcf})\end{array}$ & $\begin{array}{c}\text { Sample } \\
\text { Orientation }\end{array}$ \\
\hline 7 & Core & $\mathrm{N} / \mathrm{A}$ & $\mathrm{N} / \mathrm{A}$ & Vertical \\
\hline
\end{tabular}

Avg. $\mathrm{k}$ at $20^{\circ} \mathrm{C} \quad 4.0 \mathrm{E}-08 \mathrm{~cm} / \mathrm{sec}$

$\begin{array}{rr}\mathrm{a}_{\mathrm{a}}= & 0.76712 \mathrm{~cm}^{2} \\ \mathrm{~A} & =46.11 \mathrm{~cm}^{2} \\ \mathrm{~L} & =11.81 \mathrm{~cm} \\ \mathrm{~S}=L \mathrm{~A} & =0.25601 \mathrm{l} / \mathrm{cm}\end{array}$

$\mathrm{a}_{\mathrm{p}}=0.031416 \mathrm{~cm}^{2}$

$\mathrm{S}=\mathrm{L} / \mathrm{A}=0.256011 / \mathrm{cm}$

$\begin{aligned} \mathrm{a}_{\mathrm{p}}= & 0.031416 \mathrm{~cm}^{2} \\ \mathrm{M}_{1}= & 0.03018 \\ \mathrm{M}_{2}= & 1.04095 \\ \mathrm{C}=\mathrm{M}_{1} \mathrm{~S} /\left(\mathrm{G}_{\left.\mathrm{Hg}_{\mathrm{g}}-1\right)}=\right. & 0.0006147 \text { for } 15^{\circ} \text { to } 25^{\circ}\end{aligned}$

Remarks

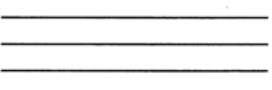

RCN: SRS256 Page 16 of 46 


\section{amec $^{\theta}$}

\section{HYDRAULIC CONDUCTIVITY}

$\begin{array}{llll}\text { Project No. } & \mathbf{6 1 5 5 - 0 8 - 0 0 3 1 . 3 4} & \text { Tested By } & \text { JEL } \\ \text { Project Name } & \text { SDU-6 Task 3 Grout Samples } & \text { Test Date } & 6 / 27 / 2012 \\ \text { Boring No. } & H 1.5-2 & \text { Reviewed By } & \text { JW } \partial \mathcal{E} \\ \text { Sample No. } & H 1.5-2 & \text { Review Date } & 8 / 1 / 2012 \\ \text { Sample Depth } & \text { N/A } & \text { Lab No. } & 11612\end{array}$

Sample Description Grout Core

ASTM D5084 - Method F (CVFH)

\begin{tabular}{|l|l|}
\hline Sample Type: & Core \\
\hline Sample Orientation: & Vertical \\
\hline Initial Water Content, \%: & 44.3 \\
\hline Wet Unit Weight, pcf: & 105.6 \\
\hline Dry Unit Weight, pcf: & 73.2 \\
\hline Compaction, \%: & N/A \\
\hline Hydraulic Conductivity, cm/sec. @20 ${ }^{\circ} \mathrm{C}$ & $\mathbf{2 . 0 E - 0 7}$ \\
\hline
\end{tabular}

Remarks: 
SRNL-STI-2012-00522

Revision 0

PERMEABILITY TEST

(ASTM D5084 - 03) (Method F, Constant Volume Falling Head)

amec $^{\theta}$

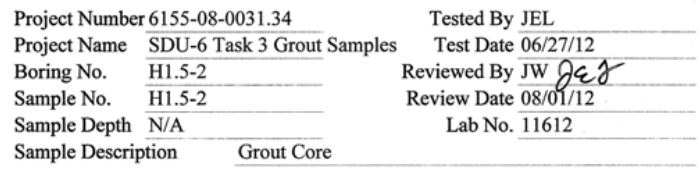

\begin{tabular}{|c|c|}
\hline Initial Sample Data & Final Sample Data \\
\hline
\end{tabular}

\begin{tabular}{|c|c|c|c|c|c|}
\hline \multicolumn{4}{|c|}{ Initial Sample Data } & \multicolumn{2}{|c|}{ Final Sample Data } \\
\hline \multicolumn{2}{|c|}{ Length, in } & \multicolumn{2}{|l|}{ Diameter, in } & Pan No. & $\mathrm{N} / \mathrm{A}$ \\
\hline Location 1 & 4.849 & Location 1 & 3.029 & Wet Soil+Pan, grams & 963.17 \\
\hline Location 2 & 4.846 & Location 2 & 3.024 & Dry Soil + Pan, grams & 667.65 \\
\hline Location3 & 4.847 & Location 3 & 3.011 & Pan Weight, grams & \\
\hline Average & 4.847 & Average & 3.021 & Moisture Content, \% & 44.3 \\
\hline Volume, in ${ }^{3}$ & 34.75 & Wet Soil + Tare, grams & 963.21 & Dry Unit Weight, pef & 73.2 \\
\hline SG Assumed & 2.40 & Tare Weight, grams & 0.00 & Saturation, \% & 101.5 \\
\hline Soil Sample Wt., g & 963.21 & Dry Soil +Tare, grams & 667.65 & Diameter, in. & N/A \\
\hline Dry UW, pef & 73.2 & Moisture Content, \% & 44.3 & Length, in. & N/A \\
\hline Saturation, \% & 101.5 & & & Volume, in ${ }^{3}$ & N/A \\
\hline
\end{tabular}

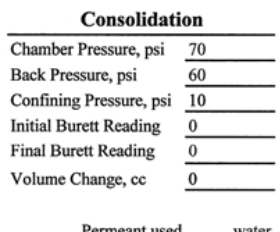

\begin{tabular}{|r|c|c|c|c|c|c|c|c|c|}
\hline $\begin{array}{c}\text { Elapsed Time } \\
(\mathrm{sec})\end{array}$ & $\begin{array}{c}\mathrm{z}_{\mathrm{o}} \\
(\mathrm{cm})\end{array}$ & $\begin{array}{c}\mathrm{za} \\
(\mathrm{cm})\end{array}$ & $\begin{array}{c}\mathrm{zb} \\
(\mathrm{cm})\end{array}$ & $\begin{array}{c}\Delta \mathrm{z}_{\mathrm{p}} \\
(\mathrm{cm})\end{array}$ & $\begin{array}{c}\text { Temp } \\
\left({ }^{\circ} \mathrm{C}\right)\end{array}$ & $\begin{array}{c}\text { Intial } \\
\text { Hydraulic } \\
\text { Gradient }\end{array}$ & $\begin{array}{c}\text { Final } \\
\text { Hydraulic } \\
\text { Gradient }\end{array}$ & $\begin{array}{c}\mathrm{k} \\
\mathrm{cm} / \mathrm{sec}\end{array}$ & $\begin{array}{c}\mathrm{k} \\
\mathrm{cm} / \mathrm{sec} \\
\mathrm{at} 20{ }^{\circ} \mathrm{C}\end{array}$ \\
\hline 640 & 1.70 & 24.10 & 19.40 & 4.70 & 22.9 & 22.9 & 17.9 & $2.46 \mathrm{E}-07$ & $2.30 \mathrm{E}-07$ \\
\hline 1080 & 1.70 & 24.40 & 18.10 & 6.30 & 22.9 & 23.2 & 16.5 & $2.02 \mathrm{E}-07$ & $1.88 \mathrm{E}-07$ \\
\hline 960 & 1.70 & 23.50 & 17.70 & 5.80 & 22.9 & 22.3 & 16.1 & $2.16 \mathrm{E}-07$ & $2.01 \mathrm{E}-07$ \\
\hline 1500 & 1.70 & 24.50 & 16.00 & 8.50 & 22.9 & 23.3 & 14.2 & $2.09 \mathrm{E}-07$ & $1.95 \mathrm{E}-07$ \\
\hline 855 & 1.70 & 25.50 & 20.00 & 5.50 & 22.9 & 24.3 & 18.5 & $2.06 \mathrm{E}-07$ & $1.92 \mathrm{E}-07$ \\
\hline 900 & 1.70 & 26.30 & 20.50 & 5.80 & 22.9 & 25.1 & 19.0 & $2.00 \mathrm{E}-07$ & $1.87 \mathrm{E}-07$ \\
\hline 720 & 1.70 & 25.00 & 20.70 & 4.30 & 22.9 & 23.8 & 19.2 & $1.89 \mathrm{E}-07$ & $1.77 \mathrm{E}-07$ \\
\hline
\end{tabular}

\begin{tabular}{|c|c|c|c|c|}
\hline No. of Trials & $\begin{array}{c}\text { Sample } \\
\text { Type }\end{array}$ & $\begin{array}{c}\text { Max. Density } \\
(\mathrm{pcf})\end{array}$ & $\begin{array}{c}\text { Compaction } \\
\%\end{array}$ & $\begin{array}{c}\text { Sample } \\
\text { Orientation }\end{array}$ \\
\hline 7 & Core & N/A & N/A & Vertical \\
\hline
\end{tabular}

Avg. $\mathrm{k}$ at $20^{\circ} \mathrm{C} \quad 2.0 \mathrm{E}-07 \mathrm{~cm} / \mathrm{sec}$

$\begin{array}{rr}\mathrm{a}_{\mathrm{a}}= & 0.76712 \mathrm{~cm}^{2} \\ \mathrm{~A} & =46.25 \mathrm{~cm}^{2} \\ \mathrm{~L} & =12.31 \mathrm{~cm} \\ \mathrm{~S}=\mathrm{L} / \mathrm{A} & =0.26618 \mathrm{l} / \mathrm{cm}\end{array}$

$a_{p}=0.031416 \mathrm{~cm}^{2}$

$\begin{array}{ll}\mathrm{M}_{1}= & 0.03018 \\ \mathrm{M}_{2}= & 1.04095\end{array}$

$\mathrm{S}=\mathrm{L} / \mathrm{A}=0.26618 \mathrm{1} / \mathrm{cm}$

$\mathrm{C}=\mathrm{M}_{1} \mathrm{~S} /\left(\mathrm{G}_{\mathrm{Hg}_{\mathrm{g}}}-1\right)=0.0006391$ for $15^{\circ}$ to $25^{\circ}$

Remarks:

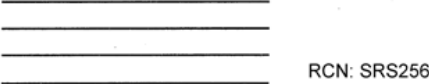

RCN: SRS 256 


\section{amec $^{\circ}$}

\section{HYDRAULIC CONDUCTIVITY}

$\begin{array}{llll}\text { Project No. } & \mathbf{6 1 5 5 - 0 8 - 0 0 3 1 . 3 4} & \text { Tested By } & \text { JEL } \\ \text { Project Name } & \text { SDU-6 Task3 Grout Samples } & \text { Test Date } & 7 / 2 / 2012 \\ \text { Boring No. } & H 1.5-3 & \text { Reviewed By } & \text { JW } G \text { C } \\ \text { Sample No. } & H 1.5-3 & \text { Review Date } & 8 / 1 / 2012 \\ \text { Sample Depth } & \text { N/A } & \text { Lab No. } & 11613\end{array}$

Sample Description Grout Core

ASTM D5084 - Method F (CVFH)

\begin{tabular}{|l|l|}
\hline Sample Type: & Core \\
\hline Sample Orientation: & Vertical \\
\hline Initial Water Content, \%: & 43.8 \\
\hline Wet Unit Weight, pcf: & 107.1 \\
\hline Dry Unit Weight, pcf: & 74.5 \\
\hline Compaction, \%: & N/A \\
\hline Hydraulic Conductivity, cm/sec. @20 C & $1.4 E-08$ \\
\hline
\end{tabular}

Remarks: 
SRNL-STI-2012-00522

Revision 0

PERMEABILITY TEST

(ASTM D5084 - 03) (Method F, Constant Volume Falling Head)

amec $^{\theta}$

Project Number 6155-08-0031.34 Tested By JEL

Project Name SDU-6 Task 3 Grout Samples Test Date 07/02/12

Boring No. H1.5-3 Reviewed By JW f $_{\varepsilon} \gamma$

Sample No. H1.5-3 Review Date 08/01/12

Sample Depth N/A Lab No. 11613

Sample Description Grout Core

\begin{tabular}{|c|r|c|r|c|r|r|}
\hline \multicolumn{4}{|c|}{ Initial Sample Data } & \multicolumn{2}{c|}{ Final Sample Data } \\
\hline \multicolumn{2}{|c|}{ Length, in } & \multicolumn{2}{|c|}{ Diameter, in } & Pan No. & N/A \\
\hline Location 1 & 4.397 & Location 1 & 3.010 & Wet Soil+Pan, grams & 880.52 \\
\hline Location 2 & 4.396 & Location 2 & 3.010 & Dry Soil + Pan, grams & 612.11 \\
\hline Location3 & 4.396 & Location 3 & 3.015 & Pan Weight, grams & \\
\hline Average & 4.396 & Average & 3.012 & Moisture Content, \% & 43.8 \\
\hline Volume, in & 31.32 & Wet Soil + Tare, grams & 880.26 & Dry Unit Weight, pef & 74.5 \\
\hline SG Assumed & 2.40 & Tare Weight, grams & 0.00 & Saturation, \% & 104.1 \\
\hline Soil Sample Wt., g & 880.26 & Dry Soil +Tare, grams & 612.11 & Diameter, in. & N/A \\
\hline Dry UW, pcf & 74.5 & Moisture Content, \% & 43.8 & Length, in. & N/A \\
\hline Saturation, \% & 104.0 & & & Volume, in & N/A \\
\hline
\end{tabular}

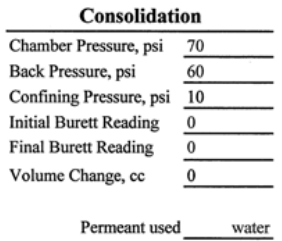

\begin{tabular}{|c|c|c|c|c|c|c|c|c|c|}
\hline $\begin{array}{c}\text { Elapsed Time } \\
(\mathrm{sec})\end{array}$ & $\begin{array}{c}\mathrm{z}_{\mathrm{o}} \\
(\mathrm{cm})\end{array}$ & $\begin{array}{c}\mathrm{za} \\
(\mathrm{cm})\end{array}$ & $\begin{array}{c}\mathrm{zb} \\
(\mathrm{cm})\end{array}$ & $\begin{array}{l}\Delta z_{\mathrm{p}} \\
(\mathrm{cm})\end{array}$ & $\begin{array}{l}\text { Temp } \\
\left({ }^{\circ} \mathrm{C}\right)\end{array}$ & $\begin{array}{c}\text { Intial } \\
\text { Hydraulic } \\
\text { Gradient } \\
\end{array}$ & \begin{tabular}{c|} 
Final \\
Hydraulic \\
Gradient \\
\end{tabular} & $\begin{array}{c}\mathrm{k} \\
\mathrm{cm} / \mathrm{sec}\end{array}$ & $\begin{array}{c}\mathrm{k} \\
\mathrm{cm} / \mathrm{sec} \\
\text { at } 20^{\circ} \mathrm{C} \\
\end{array}$ \\
\hline 4200 & 1.70 & 24.00 & 21.10 & 2.90 & 23.0 & 25.1 & 21.7 & $2.02 \mathrm{E}-08$ & $1.88 \mathrm{E}-08$ \\
\hline 720 & 1.70 & 25.00 & 24.50 & 0.50 & 23.0 & 26.2 & 25.6 & $1.83 \mathrm{E}-08$ & $1.70 \mathrm{E}-08$ \\
\hline 1620 & 1.70 & 25.00 & 24.10 & 0.90 & 23.0 & 26.2 & 25.2 & $1.48 \mathrm{E}-08$ & $1.38 \mathrm{E}-08$ \\
\hline 2700 & 1.70 & 25.00 & 23.70 & 1.30 & 23.0 & 26.2 & 24.7 & $1.29 \mathrm{E}-08$ & $1.20 \mathrm{E}-08$ \\
\hline 3660 & 1.70 & 25.00 & 23.20 & 1.80 & 23.0 & 26.2 & 24.1 & $1.34 \mathrm{E}-08$ & $1.24 \mathrm{E}-08$ \\
\hline 4920 & 1.70 & 25.00 & 22.60 & 2.40 & 23.0 & 26.2 & 23.4 & $1.34 \mathrm{E}-08$ & $1.25 \mathrm{E}-08$ \\
\hline
\end{tabular}

\begin{tabular}{|c|c|c|c|c|}
\hline No. of Trials & $\begin{array}{c}\text { Sample } \\
\text { Type }\end{array}$ & $\begin{array}{c}\text { Max. Density Compaction } \\
\text { (pcf) }\end{array}$ & $\begin{array}{c}\text { Sample } \\
\text { Orientation }\end{array}$ \\
\hline
\end{tabular}

Avg. $\mathrm{k}$ at $20^{\circ} \mathrm{C} \quad 1.4 \mathrm{E}-08 \mathrm{~cm} / \mathrm{sec}$

$\begin{aligned} \mathrm{a}_{\mathrm{a}} & =0.76712 \mathrm{~cm}^{2} \\ \mathrm{~A} & =\quad 45.96 \mathrm{~cm}^{2} \\ \mathrm{~L} & =11.17 \mathrm{~cm} \\ \mathrm{~S}=\mathrm{L} / \mathrm{A} & =0.24297 \mathrm{l} / \mathrm{cm}\end{aligned}$

$\begin{aligned} \mathrm{a}_{\mathrm{p}}= & 0.031416 \mathrm{~cm}^{2} \\ \mathrm{M}_{1}= & 0.03018 \\ \mathrm{M}_{2}= & 1.04095\end{aligned}$

$S=L / A=0.242971 / \mathrm{cm}$

$\mathrm{C}=\mathrm{M}_{1} \mathrm{~S} /\left(\mathrm{G}_{\mathrm{Hg}}-1\right)=0.0005834$ for $15^{\circ}$ to $25^{\circ}$

Remarks:

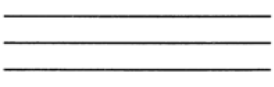

RCN: SRS256 Page 20 of 46 


\section{amec $^{\circ}$}

\section{HYDRAULIC CONDUCTIVITY}

$\begin{array}{llll}\text { Project No. } & \text { 6155-08-0031.34 } & \text { Tested By } & \text { JEL } \\ \text { Project Name } & \text { SDU-6 Task 3 Grout Samples } & \text { Test Date } & 7 / 2 / 2012 \\ \text { Boring No. } & \text { H1.0-1 } & \text { Reviewed By } & \text { JWg氏f } \\ \text { Sample No. } & \text { H1.0-1 } & \text { Review Date } & 8 / 1 / 2012 \\ \text { Sample Depth } & \text { N/A } & \text { Lab No. } & 11614\end{array}$

Sample Description Grout Core

ASTM D5084 - Method F (CVFH)

\begin{tabular}{|l|l|}
\hline Sample Type: & Core \\
\hline Sample Orientation: & Vertical \\
\hline Initial Water Content, \%: & 44.3 \\
\hline Wet Unit Weight, pcf: & 106.0 \\
\hline Dry Unit Weight, pcf: & 73.5 \\
\hline Compaction, \%: & N/A \\
\hline Hydraulic Conductivity, cm/sec. @20 C & $\mathbf{4 . 0 E - 0 8}$ \\
\hline
\end{tabular}

Remarks: 
PERMEABILITY TEST

(ASTM D5084 - 03) (Method F, Constant Volume Falling Head)

Project Number 6155-08-0031.34 Tested By JEL

Project Name SDU-6 Task 3 Grout Samples Test Date 07/02/12

Boring No. H1.0-1 Reviewed By JW Oa

Sample No. H1.0-1 Review Date 08/0)/12

Sample Depth N/A Lab No. 11614

Sample Description Grout Core

\begin{tabular}{|c|r|c|r|c|r|r|}
\hline \multicolumn{4}{|c|}{ Initial Sample Data } & \multicolumn{2}{c|}{ Final Sample Data } \\
\hline \multicolumn{2}{|c|}{ Length, in } & \multicolumn{2}{|c|}{ Diameter, in } & Pan No. & N/A \\
\hline Location 1 & 4.654 & Location 1 & 3.033 & Wet Soil+Pan, grams & 924.12 \\
\hline Location 2 & 4.646 & Location 2 & 2.993 & Dry Soil + Pan, grams & 640.32 \\
\hline Location3 & 4.650 & Location 3 & 3.020 & Pan Weight, grams & 0 \\
\hline Average & 4.650 & Average & 3.015 & Moisture Content, \% & 44.3 \\
\hline Volume, in & 33.21 & Wet Soil + Tare, grams & 924.11 & Dry Unit Weight, pcf & 73.5 \\
\hline SG Assumed & 2.40 & Tare Weight, grams & 0.00 & Saturation, \% & 102.4 \\
\hline Soil Sample Wt. g & 924.11 & Dry Soil +Tare, grams & 640.32 & Diameter, in. & N/A \\
\hline Dry UW, pef & 73.5 & Moisture Content, \% & 44.3 & Length, in. & N/A \\
\hline Saturation, \% & 102.4 & & & Volume, in & N/A \\
\hline
\end{tabular}

\begin{tabular}{|r|c|c|c|r|r|r|r|r|r|}
\hline $\begin{array}{c}\text { Elapsed Time } \\
(\mathrm{sec})\end{array}$ & $\begin{array}{c}\mathrm{z}_{\mathrm{o}} \\
(\mathrm{cm})\end{array}$ & $\begin{array}{c}\mathrm{za} \\
(\mathrm{cm})\end{array}$ & $\begin{array}{c}\mathrm{zb} \\
(\mathrm{cm})\end{array}$ & $\begin{array}{c}\Delta \mathrm{z}_{\mathrm{p}} \\
(\mathrm{cm})\end{array}$ & $\begin{array}{c}\text { Temp } \\
\left({ }^{\circ} \mathrm{C}\right)\end{array}$ & $\begin{array}{c}\text { Intial } \\
\text { Hydraulic } \\
\text { Gradient }\end{array}$ & $\begin{array}{c}\text { Final } \\
\text { Hydraulic } \\
\text { Gradient }\end{array}$ & $\begin{array}{c}\mathrm{k} \\
\mathrm{cm} / \mathrm{sec}\end{array}$ & $\begin{array}{c}\mathrm{k} \\
\mathrm{cm} / \mathrm{sec} \\
\mathrm{at} 20^{\circ} \mathrm{C}\end{array}$ \\
\hline 2040 & 1.70 & 25.00 & 21.50 & 3.50 & 23.0 & 24.8 & 20.9 & $5.13 \mathrm{E}-08$ & $4.78 \mathrm{E}-08$ \\
\hline 4620 & 1.70 & 25.00 & 18.50 & 6.50 & 23.0 & 24.8 & 17.6 & $4.57 \mathrm{E}-08$ & $4.26 \mathrm{E}-08$ \\
\hline 720 & 1.70 & 25.00 & 23.90 & 1.10 & 23.0 & 24.8 & 23.6 & $4.31 \mathrm{E}-08$ & $4.01 \mathrm{E}-08$ \\
\hline 1620 & 1.70 & 25.00 & 22.70 & 2.30 & 23.0 & 24.8 & 22.2 & $4.12 \mathrm{E}-08$ & $3.84 \mathrm{E}-08$ \\
\hline 2760 & 1.70 & 25.00 & 21.40 & 3.60 & 23.0 & 24.8 & 20.8 & $3.91 \mathrm{E}-08$ & $3.64 \mathrm{E}-08$ \\
\hline 3660 & 1.70 & 25.00 & 20.30 & 4.70 & 23.0 & 24.8 & 19.6 & $3.96 \mathrm{E}-08$ & $3.69 \mathrm{E}-08$ \\
\hline 5040 & 1.70 & 25.00 & 19.00 & 6.00 & 23.0 & 24.8 & 18.2 & $3.81 \mathrm{E}-08$ & $3.55 \mathrm{E}-08$ \\
\hline
\end{tabular}

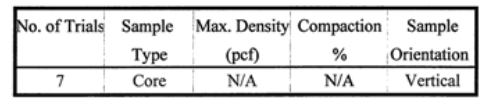

Avg. $\mathrm{k}$ at $20^{\circ} \mathrm{C} \quad 4.0 \mathrm{E}-08 \mathrm{~cm} / \mathrm{sec}$

\begin{tabular}{|c|c|c|c|}
\hline$a_{2}=$ & $0.76712 \mathrm{~cm}^{2}$ & $a_{p}=$ & $0.031416 \mathrm{~cm}^{2}$ \\
\hline A & $46.07 \mathrm{~cm}^{2}$ & $M_{1}=$ & 0.03018 \\
\hline $\mathrm{L}=$ & $11.81 \mathrm{~cm}$ & $\mathrm{M}_{2}=$ & 1.04095 \\
\hline
\end{tabular}

$\mathrm{S}=\mathrm{L} / \mathrm{A}=0.25636 \mathrm{H} / \mathrm{cm}$

$\mathrm{C}=\mathrm{M}_{1} \mathrm{~S} /\left(\mathrm{G}_{\mathrm{Hg}}-1\right)=0.0006155$ for $15^{\circ}$ to $25^{\circ}$ amec $^{\theta}$

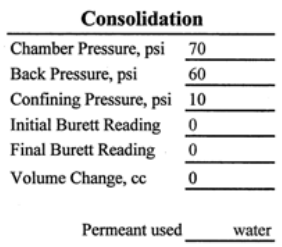

Permeant used

8




\section{$\operatorname{amec}^{\theta}$}

\section{HYDRAULIC CONDUCTIVITY}

$\begin{array}{llll}\text { Project No. } & \text { 6155-08-0031.34 } & \text { Tested By } & \text { JEL } \\ \text { Project Name } & \text { SDU-6 Task 3 Grout Samples } & \text { Test Date } & 7 / 2 / 2012 \\ \text { Boring No. } & H 1.0-2 & \text { Reviewed By } & \text { JW } \partial \varepsilon \text { Y } \\ \text { Sample No. } & \text { H1.0-2 } & \text { Review Date } & 8 / 1 / 2012 \\ \text { Sample Depth } & \text { N/A } & \text { Lab No. } & \mathbf{1 1 6 1 5}\end{array}$

Sample Description Grout Core

ASTM D5084 - Method F (CVFH)

\begin{tabular}{|l|l|}
\hline Sample Type: & Core \\
\hline Sample Orientation: & Vertical \\
\hline Initial Water Content, \%: & 44.1 \\
\hline Wet Unit Weight, pcf: & 106.1 \\
\hline Dry Unit Weight, pcf: & 73.7 \\
\hline Compaction, \%: & N/A \\
\hline Hydraulic Conductivity, cm/sec. @20 C & $\mathbf{2 . 4 E - 0 7}$ \\
\hline
\end{tabular}

Remarks: 
SRNL-STI-2012-00522

Revision 0

PERMEABILITY TEST

(ASTM D5084 - 03) (Method F, Constant Volume Falling Head)

amec $^{\theta}$

Project Number 6155-08-0031.34

Tested By JEL

Project Name SDU-6 Task 3 Grout Samples Test Date 07/02/12

Boring No. H1.0-2 Reviewed By JW /q \&

Sample No. H1.0-2 Review Date 08/81/12

$\begin{array}{lll}\text { Sample Depth N/A } & & \text { Lab No. } 11615 \\ \text { Sample Description } & \text { Grout Core }\end{array}$

\begin{tabular}{|c|r|c|r|c|r|r|}
\hline \multicolumn{4}{|c|}{ Initial Sample Data } & \multicolumn{2}{c|}{ Final Sample Data } \\
\hline \multicolumn{2}{|c|}{ Length, in } & \multicolumn{2}{c|}{ Diameter, in } & Pan No. & N/A \\
\hline Location 1 & 4.505 & Location 1 & 3.011 & Wet Soil+Pan, grams & 894.44 \\
\hline Location 2 & 4.482 & Location 2 & 3.017 & Dry Soil + Pan, grams & 619.38 \\
\hline Location3 & 4.484 & Location 3 & 3.013 & Pan Weight, grams & 0 \\
\hline Average & 4.490 & Average & 3.014 & Moisture Content, \% & 44.4 \\
\hline Volume, in & 32.03 & Wet Soil + Tare, grams & 892.30 & Dry Unit Weight, pef & 73.7 \\
\hline SG Assumed & 2.40 & Tare Weight, grams & 0.00 & Saturation, \% & 103.2 \\
\hline Soil Sample Wt., g & 892.30 & Dry Soil +Tare, grams & 619.38 & Diameter, in. & N/A \\
\hline Dry UW, pcf & 73.7 & Moisture Content, \% & 44.1 & Length, in. & N/A \\
\hline Saturation, \% & 102.4 & & & Volume, in & N/A \\
\hline
\end{tabular}

\begin{tabular}{|c|c|}
\hline \multicolumn{2}{|c|}{ Consolidation } \\
\hline Chamber Pressure, psi & 70 \\
\hline Back Pressure, psi & 60 \\
\hline Confining Pressure, psi & 10 \\
\hline Initial Burett Reading & 0 \\
\hline Final Burett Reading & 0 \\
\hline Volume Change, cc & 0 \\
\hline
\end{tabular}

\begin{tabular}{|r|c|c|c|c|c|c|c|c|c|}
\hline $\begin{array}{c}\text { Elapsed Time } \\
(\mathrm{sec})\end{array}$ & $\begin{array}{c}\mathrm{z}_{\mathrm{o}} \\
(\mathrm{cm})\end{array}$ & $\begin{array}{c}\mathrm{za} \\
(\mathrm{cm})\end{array}$ & $\begin{array}{c}\mathrm{zb} \\
(\mathrm{cm})\end{array}$ & $\begin{array}{c}\Delta \mathrm{s}_{\mathrm{p}} \\
(\mathrm{cm})\end{array}$ & $\begin{array}{c}\text { Temp } \\
\left({ }^{\circ} \mathrm{C}\right)\end{array}$ & $\begin{array}{c}\text { Intial } \\
\text { Hydraulic } \\
\text { Gradient }\end{array}$ & $\begin{array}{c}\text { Final } \\
\text { Hydraulic } \\
\text { Gradient }\end{array}$ & $\begin{array}{c}\mathrm{k} \\
\mathrm{cm} / \mathrm{sec}\end{array}$ & $\begin{array}{c}\mathrm{k} \\
\mathrm{cm} / \mathrm{sec} \\
\mathrm{at} 20^{\circ} \mathrm{C}\end{array}$ \\
\hline 540 & 1.70 & 25.00 & 19.50 & 5.50 & 23.0 & 25.7 & 19.4 & $3.11 \mathrm{E}-07$ & $2.89 \mathrm{E}-07$ \\
\hline 720 & 1.70 & 25.00 & 19.10 & 5.90 & 23.0 & 25.7 & 18.9 & $2.53 \mathrm{E}-07$ & $2.35 \mathrm{E}-07$ \\
\hline 1080 & 1.70 & 25.00 & 17.00 & 8.00 & 23.0 & 25.7 & 16.5 & $2.44 \mathrm{E}-07$ & $2.27 \mathrm{E}-07$ \\
\hline 600 & 1.70 & 25.00 & 20.30 & 4.70 & 23.0 & 25.7 & 20.3 & $2.34 \mathrm{E}-07$ & $2.18 \mathrm{E}-07$ \\
\hline 540 & 1.70 & 25.00 & 20.20 & 4.80 & 23.0 & 25.7 & 20.2 & $2.66 \mathrm{E}-07$ & $2.48 \mathrm{E}-07$ \\
\hline 1080 & 1.70 & 25.00 & 16.70 & 8.30 & 23.0 & 25.7 & 16.2 & $2.55 \mathrm{E}-07$ & $2.38 \mathrm{E}-07$ \\
\hline 480 & 1.70 & 25.00 & 20.70 & 4.30 & 23.0 & 25.7 & 20.7 & $2.64 \mathrm{E}-07$ & $2.46 \mathrm{E}-07$ \\
\hline
\end{tabular}

\begin{tabular}{|c|c|c|c|c|}
\hline No. of Trials & Sample & Max. Density & Compaction & Sample \\
\hline 7 & Core & N/A & $\mathrm{N} / \mathrm{A}$ & Vertical \\
\hline
\end{tabular}

Avg. $\mathrm{k}$ at $20^{\circ} \mathrm{C} \quad 2.4 \mathrm{E}-07 \mathrm{~cm} / \mathrm{sec}$

$\begin{array}{rr}\mathrm{a}_{\mathrm{a}}= & 0.76712 \mathrm{~cm}^{2} \\ \mathrm{~A} & =46.02 \mathrm{~cm}^{2} \\ \mathrm{~L} & =11.41 \mathrm{~cm} \\ \mathrm{~S}=\mathrm{LA} & =0.24784 \mathrm{1} / \mathrm{cm}\end{array}$

$\mathrm{a}_{\mathrm{p}}=0.031416 \mathrm{~cm}^{2}$

$\mathrm{L}=11.41 \mathrm{~cm}$

$\mathrm{M}_{1}=0.03018$

$\mathrm{S}=\mathrm{L} / \mathrm{A}=0.24784 \mathrm{1} / \mathrm{cm}$

$\mathrm{C}=\mathrm{M}_{\mathrm{l}} \mathrm{S} /\left(\mathrm{G}_{\mathrm{Hg}}-1\right)=0.0005950$ for $15^{\circ}$ to $25^{\circ}$

Remarks:

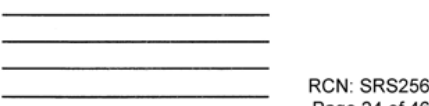

RCN: SRS256 


\section{amec $^{\theta}$}

\section{HYDRAULIC CONDUCTIVITY}

$\begin{array}{llll}\text { Project No. } & \text { 6155-08-0031.34 } & \text { Tested By } & \text { JEL } \\ \text { Project Name } & \text { SDU-6 Task 3 Grout Samples } & \text { Test Date } & \text { 7/2/2012 } \\ \text { Boring No. } & \text { H1.0-3 } & \text { Reviewed By } & \text { JWGG } \\ \text { Sample No. } & \text { H1.0-3 } & \text { Review Date } & 8 / 1 / 2012 \\ \text { Sample Depth } & \text { N/A } & \text { Lab No. } & \mathbf{1 1 6 1 6}\end{array}$

Sample Description Grout Core

ASTM D5084 - Method F (CVFH)

\begin{tabular}{|l|l|}
\hline Sample Type: & Core \\
\hline Sample Orientation: & Vertical \\
\hline Initial Water Content, \%: & 43.8 \\
\hline Wet Unit Weight, pcf: & 105.9 \\
\hline Dry Unit Weight, pcf: & 73.6 \\
\hline Compaction, \%: & N/A \\
\hline Hydraulic Conductivity, cm/sec. @20 & \\
\hline
\end{tabular}

Remarks:

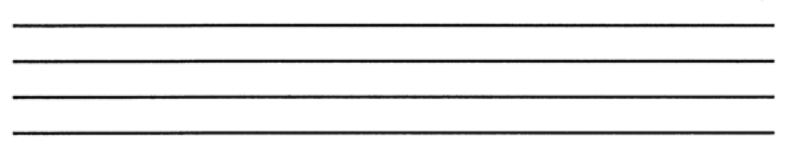


SRNL-STI-2012-00522

Revision 0

PERMEABILITY TEST

(ASTM D5084 - 03) (Method F, Constant Volume Falling Head)

amec $^{\theta}$

Project Number 6155-08-0031.34

Tested By JEL

Project Name SDU-6 Task 3 Grout Samples Test Date 07/02/12

Boring No. H1.0-3 Reviewed By JW $/ \varepsilon f$

Sample No. H1.0-3 Review Date 08/01/12

Sample Depth N/A Lab No. 11616

Sample Description Grout Core

Lab No. 11616

\begin{tabular}{|c|r|c|r|c|c|}
\hline \multicolumn{4}{|c|}{ Initial Sample Data } & \multicolumn{2}{c|}{ Final Sample Data } \\
\hline \multicolumn{2}{|c|}{ Length, in } & \multicolumn{2}{|c|}{ Diameter, in } & Pan No. & N/A \\
\hline Location 1 & 4.507 & Location 1 & 3.011 & Wet Soil+Pan, grams & 900.01 \\
\hline Location 2 & 4.504 & Location 2 & 3.038 & Dry Soil + Pan, grams & 626.07 \\
\hline Location3 & 4.495 & Location 3 & 3.032 & Pan Weight, grams & \\
\hline Average & 4.502 & Average & 3.027 & Moisture Content, \% & 43.8 \\
\hline Volume, in & 32.40 & Wet Soil + Tare, grams & 900.41 & Dry Unit Weight, pcf & 73.6 \\
\hline SG Assumed & 2.40 & Tare Weight, grams & 0.00 & Saturation, \% & 101.5 \\
\hline Soil Sample Wt., g & 900.41 & Dry Soil +Tare, grams & 626.07 & Diameter, in. & N/A \\
\hline Dry UW, pcf & 73.6 & Moisture Content, \% & 43.8 & Length, in. & N/A \\
\hline Saturation, \% & 101.7 & & & Volume, in & N/A \\
\hline
\end{tabular}

Consolidation Chamber Pressure, psi 70 Back Pressure, psi Confining Pressure, psi 10

Initial Burett Reading

Final Burett Reading

Volume Change, cc

Permeant used

\begin{tabular}{ll}
$\frac{70}{60}$ \\
\hline 10 \\
\hline 0 \\
\hline 0 \\
\hline 0 & \\
\hline & \\
\hline
\end{tabular}

\begin{tabular}{|r|c|c|c|c|c|c|c|c|c|}
\hline $\begin{array}{c}\text { Elapsed Time } \\
(\mathrm{sec})\end{array}$ & $\begin{array}{c}\mathrm{z}_{\mathrm{v}} \\
(\mathrm{cm})\end{array}$ & $\begin{array}{c}\mathrm{za} \\
(\mathrm{cm})\end{array}$ & $\begin{array}{c}\mathrm{zb} \\
(\mathrm{cm})\end{array}$ & $\begin{array}{c}\Delta \mathrm{z}_{\mathrm{p}} \\
(\mathrm{cm})\end{array}$ & $\begin{array}{c}\text { Temp } \\
\left({ }^{\circ} \mathrm{C}\right)\end{array}$ & $\begin{array}{c}\text { Intial } \\
\text { Hydraulic } \\
\text { Gradient }\end{array}$ & $\begin{array}{c}\text { Final } \\
\text { Hydraulic } \\
\text { Gradient }\end{array}$ & $\begin{array}{c}\mathrm{k} \\
\mathrm{cm} / \mathrm{sec}\end{array}$ & $\begin{array}{c}\mathrm{k} \\
\mathrm{cm} / \mathrm{sec} \\
\mathrm{at} 20{ }^{\circ} \mathrm{C}\end{array}$ \\
\hline 540 & 1.70 & 25.00 & 20.70 & 4.30 & 23.0 & 25.6 & 20.7 & $2.34 \mathrm{E}-07$ & $2.18 \mathrm{E}-07$ \\
\hline 720 & 1.70 & 25.50 & 20.60 & 4.90 & 23.0 & 26.2 & 20.6 & $1.98 \mathrm{E}-07$ & $1.84 \mathrm{E}-07$ \\
\hline 900 & 1.70 & 25.00 & 19.00 & 6.00 & 23.0 & 25.6 & 18.7 & $2.05 \mathrm{E}-07$ & $1.91 \mathrm{E}-07$ \\
\hline 660 & 1.70 & 25.80 & 20.70 & 5.10 & 23.0 & 26.5 & 20.7 & $2.23 \mathrm{E}-07$ & $2.08 \mathrm{E}-07$ \\
\hline 540 & 1.70 & 25.00 & 22.00 & 3.00 & 23.0 & 25.6 & 22.2 & $1.58 \mathrm{E}-07$ & $1.47 \mathrm{E}-07$ \\
\hline 1080 & 1.70 & 25.00 & 20.20 & 4.80 & 23.0 & 25.6 & 20.1 & $1.32 \mathrm{E}-07$ & $1.23 \mathrm{E}-07$ \\
\hline 540 & 1.70 & 25.00 & 21.50 & 3.50 & 23.0 & 25.6 & 21.6 & $1.86 \mathrm{E}-07$ & $1.73 \mathrm{E}-07$ \\
\hline
\end{tabular}

\begin{tabular}{|c|c|c|c|c|}
\hline No. of Trials & $\begin{array}{c}\text { Sample } \\
\text { Type }\end{array}$ & $\begin{array}{c}\text { Max. Density } \\
(\mathrm{pcf})\end{array}$ & $\begin{array}{c}\text { Compaction } \\
\%\end{array}$ & $\begin{array}{c}\text { Sample } \\
\text { Orientation }\end{array}$ \\
\hline 7 & Core & N/A & N/A & Vertical \\
\hline
\end{tabular}

Avg. $\mathrm{k}$ at $20^{\circ} \mathrm{C} \quad 1.8 \mathrm{E}-07 \mathrm{~cm} / \mathrm{sec}$

$\begin{aligned} \mathrm{a}_{\mathrm{a}} & =0.76712 \mathrm{~cm}^{2} \\ \mathrm{~A} & =-46.43 \mathrm{~cm}^{2} \\ \mathrm{~L} & =\frac{11.44 \mathrm{~cm}}{\mathrm{~S}=\mathrm{L} / \mathrm{A}}=0.24630 \mathrm{l} / \mathrm{cm}\end{aligned}$

$\mathrm{a}_{\mathrm{p}}=0.031416 \mathrm{~cm}^{2}$

$\mathrm{M}_{1}=0.03018$

$\mathrm{M}_{2}=1.04095$

$\mathrm{S}=\mathrm{L} / \mathrm{A}=0.24630 \mathrm{I} / \mathrm{cm}$

$\mathrm{C}=\mathrm{M}_{1} \mathrm{~S} /\left(\mathrm{G}_{\mathrm{Hg}}-1\right)=0.0005913$ for $15^{\circ}$ to $25^{\circ}$

Remarks:

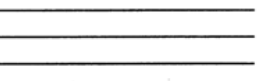

RCN: SRS256 Page 26 of 46 


\section{amec $^{\theta}$}

\section{HYDRAULIC CONDUCTIVITY}

$\begin{array}{llll}\text { Project No. } & \mathbf{6 1 5 5 - 0 8 - 0 0 3 1 . 3 4} & \text { Tested By } & \text { JEL } \\ \text { Project Name } & \text { SDU-6 Task 3 Grout Samples } & \text { Test Date } & 7 / 10 / 2012 \\ \text { Boring No. } & \text { V0.5-1 } & \text { Reviewed By } & \text { JW } 6 \text { \&f } \\ \text { Sample No. } & \text { V0.5-1 } & \text { Review Date } & 8 / 1 / 2012 \\ \text { Sample Depth } & \text { N/A } & \text { Lab No. } & 11617\end{array}$

Sample Description Grout Core

ASTM D5084 - Method F (CVFH)

\begin{tabular}{|l|l|}
\hline Sample Type: & Core \\
\hline Sample Orientation: & Vertical \\
\hline Initial Water Content, \%: & 43.7 \\
\hline Wet Unit Weight, pcf: & 106.4 \\
\hline Dry Unit Weight, pcf: & 74.0 \\
\hline Compaction, \%: & N/A \\
\hline Hydraulic Conductivity, cm/sec. @20 ${ }^{\circ} \mathrm{C}$ & $\mathbf{1 . 6 E - 0 9}$ \\
\hline
\end{tabular}

Remarks: Top layey separated during the test. 
SRNL-STI-2012-00522

Revision 0

PERMEABILITY TEST

(ASTM D5084 - 03) (Method F, Constant Volume Falling Head)

$\operatorname{amec}^{\theta}$

Project Number 6155-08-0031.34 Tested By JEL

Project Name SDU-6 Task 3 Grout Samples Test Date 07/10/12

Boring No. V0.5-1 Reviewed By JW gदf

Sample No. V0.5-1 Review Date 08/01/12

$\begin{array}{lll}\text { Sample Depth N/A } & \text { Lab No. } 11617 \\ \text { Sample Description } & \text { Grout Core }\end{array}$

\begin{tabular}{|c|r|c|r|c|r|}
\hline \multicolumn{3}{|c|}{ Initial Sample Data } & \multicolumn{2}{c|}{ Final Sample Data } \\
\hline Length, in & \multicolumn{2}{|c|}{ Diameter, in } & Pan No. & N/a \\
\hline Location 1 & 5.513 & Location 1 & 3.041 & Wet Soil+Pan, grams & 1116.33 \\
\hline Location 2 & 5.511 & Location 2 & 3.048 & Dry Soil + Pan, grams & 776.43 \\
\hline Location3 3 & 5.521 & Location 3 & 3.024 & Pan Weight, grams & 0 \\
\hline Average & 5.515 & Average & 3.038 & Moisture Content, \% & 43.8 \\
\hline Volume, in & 39.97 & Wet Soil + Tare, grams & 1115.87 & Dry Unit Weight, pcf & 74.0 \\
\hline SG Assumed & 2.40 & Tare Weight, grams & 0.00 & Saturation, \% & 102.6 \\
\hline Soil Sample Wt., g & 1115.87 & Dry Soil +Tare, grams & 776.43 & Diameter, in. & N/A \\
\hline Dry UW, pcf & 74.0 & Moisture Content, \% & 43.7 & Length, in. & N/A \\
\hline Saturation, \% & 102.5 & & & Volume, in & N/A \\
\hline
\end{tabular}

Consolidation

\begin{tabular}{ll}
\hline Chamber Pressure, psi & 70 \\
Back Pressure, psi & 60 \\
Confining Pressure, psi & 10 \\
Initial Burett Reading & 0 \\
Final Burett Reading & 0 \\
Volume Change, cc & 0
\end{tabular}

Permeant used water

\begin{tabular}{|c|c|c|c|c|c|c|c|c|c|}
\hline $\begin{array}{l}\text { Elapsed Time } \\
\quad(\mathrm{sec})\end{array}$ & $\begin{array}{c}\mathrm{z}_{\mathrm{o}} \\
(\mathrm{cm})\end{array}$ & $\begin{array}{c}\mathrm{za} \\
(\mathrm{cm})\end{array}$ & $\begin{array}{c}\mathrm{zb} \\
(\mathrm{cm})\end{array}$ & $\begin{array}{l}\Delta \mathrm{z}_{\mathrm{p}} \\
(\mathrm{cm})\end{array}$ & $\begin{array}{l}\text { Temp } \\
\left({ }^{\circ} \mathrm{C}\right)\end{array}$ & $\begin{array}{c}\text { Intial } \\
\text { Hydraulic } \\
\text { Gradient } \\
\end{array}$ & $\begin{array}{c}\text { Final } \\
\text { Hydraulic } \\
\text { Gradient } \\
\end{array}$ & $\begin{array}{c}\mathrm{k} \\
\mathrm{cm} / \mathrm{sec}\end{array}$ & $\begin{array}{c}\mathrm{k} \\
\mathrm{cm} / \mathrm{sec} \\
\text { at } 20^{\circ} \mathrm{C}\end{array}$ \\
\hline 4980 & 2.20 & 26.40 & 25.95 & 0.45 & 22.8 & 21.7 & 21.3 & 2.82E-09 & $2.64 \mathrm{E}-09$ \\
\hline 9600 & 2.20 & 26.40 & 25.85 & 0.55 & 22.9 & 21.7 & 21.2 & $1.79 \mathrm{E}-09$ & $1.67 \mathrm{E}-09$ \\
\hline 19680 & 2.20 & 26.40 & 25.70 & 0.70 & 22.9 & 21.7 & 21.1 & $1.12 \mathrm{E}-09$ & $1.04 \mathrm{E}-09$ \\
\hline 7440 & 2.20 & 26.70 & 26.20 & 0.50 & 22.3 & 22.0 & 21.5 & 2.08E-09 & $1.97 \mathrm{E}-09$ \\
\hline 14580 & 2.20 & 26.70 & 26.10 & 0.60 & 22.9 & 22.0 & 21.4 & $1.27 \mathrm{E}-09$ & $1.19 \mathrm{E}-09$ \\
\hline 21900 & 2.20 & 26.70 & 25.90 & 0.80 & 22.6 & 22.0 & 21.2 & $1.14 \mathrm{E}-09$ & $1.07 \mathrm{E}-09$ \\
\hline
\end{tabular}

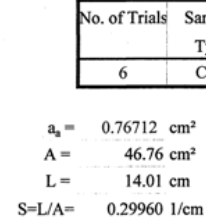

Sample $\quad$ Max. Density Compaction $\quad$ Sample

\begin{tabular}{c|c|c|c|} 
Type & $(\mathrm{pcf})$ & $\%$ & Orientation \\
\hline Core & N/A & N/A & Vertical \\
\hline
\end{tabular}

Avg. $\mathrm{k}$ at $20^{\circ} \mathrm{C} \quad \underline{1.6 \mathrm{E}-09} \mathrm{~cm} / \mathrm{sec}$

Remarks: Top layey separated during the test.

$\mathrm{S}=\mathrm{L} / \mathrm{A}=0.29960 \mathrm{l} / \mathrm{cm}$

$\mathrm{a}=0.031416 \mathrm{~cm}^{2}$

$\mathrm{M}_{1}=0.03018$

$\mathrm{M}_{2}=\quad 1.04095$

$\mathrm{C}=\mathrm{M}_{1} \mathrm{~S} /\left(\mathrm{G}_{\mathrm{Hg}}-1\right)=0.0007193$ for $15^{\circ}$ to $25^{\circ}$

RCN: SRS256 Page 28 of 46 


\section{$\operatorname{amec}^{\infty}$}

\section{HYDRAULIC CONDUCTIVITY}

\begin{tabular}{|c|c|c|c|}
\hline Project No. & $6155-08-0031.34$ & Tested By & $J E L$ \\
\hline Project Name & SDU-6 Task 3 Grout Samples & Test Date & $7 / 10 / 2012$ \\
\hline Boring No. & $V 0.5-2$ & Reviewed By & JWg氏y \\
\hline Sample No. & $V 0.5-2$ & Review Date & $8 / 1 / 2012$ \\
\hline Sample Depth & $N / A$ & Lab No. & 11618 \\
\hline
\end{tabular}

Sample Description Grout Core

ASTM D5084 - Method F (CVFH)

\begin{tabular}{|l|l|}
\hline Sample Type: & Core \\
\hline Sample Orientation: & Vertical \\
\hline Initial Water Content, \%: & 44.9 \\
\hline Wet Unit Weight, pcf: & 105.9 \\
\hline Dry Unit Weight, pcf: & 73.1 \\
\hline Compaction, \%: & N/A \\
\hline Hydraulic Conductivity, cm/sec. @20 C & $\mathbf{4 . 2 E - 0 9}$ \\
\hline
\end{tabular}

Remarks: 
SRNL-STI-2012-00522

Revision 0

PERMEABILITY TEST

(ASTM D5084 - 03) (Method F, Constant Volume Falling Head)

Project Number 6155-08-0031.34

Boring No. V0.5-2 Reviewed By JW $8 \&$ f

Sample No. V0.5-2 Review Date 08/01/12

Sample Depth N/A Lab No. 11618

Sample Description Grout Core

\begin{tabular}{|c|r|c|r|c|r|r|}
\hline \multicolumn{4}{|c|}{ Initial Sample Data } & \multicolumn{2}{c|}{ Final Sample Data } \\
\hline \multicolumn{2}{|c|}{ Length, in } & \multicolumn{2}{|c|}{ Diameter, in } & Pan No. & N/A \\
\hline Location 1 & 5.418 & Location 1 & 3.047 & Wet Soil+Pan, grams & 1094.29 \\
\hline Location 2 & 5.412 & Location 2 & 3.031 & Dry Soil + Pan, grams & 754.76 \\
\hline Location3 & 5.409 & Location 3 & 3.049 & Pan Weight, grams & 0 \\
\hline Average & 5.413 & Average & 3.042 & Moisture Content, \% & 45.0 \\
\hline Volume, in & 39.35 & Wet Soil + Tare, grams & 1093.90 & Dry Unit Weight, pcf & 73.1 \\
\hline SG Assumed & 2.40 & Tare Weight, grams & 0.00 & Saturation, \% & 102.9 \\
\hline Soil Sample Wt., g & 1093.90 & Dry Soil +Tare, grams & 754.76 & Diameter, in. & N/A \\
\hline Dry UW, pcf & 73.1 & Moisture Content, \% & 44.9 & Length, in. & N/A \\
\hline Saturation, \% & 102.8 & & & Volume, in & N/A \\
\hline
\end{tabular}

\begin{tabular}{|r|r|r|r|r|r|r|r|r|r|}
\hline \begin{tabular}{|} 
Elapsed Time \\
$(\mathrm{sec})$
\end{tabular} & $\begin{array}{c}\mathrm{z}_{\mathrm{o}} \\
(\mathrm{cm})\end{array}$ & $\begin{array}{c}\mathrm{za} \\
(\mathrm{cm})\end{array}$ & $\begin{array}{c}\mathrm{zb} \\
(\mathrm{cm})\end{array}$ & $\begin{array}{c}\Delta \mathrm{z}_{\mathrm{p}} \\
(\mathrm{cm})\end{array}$ & $\begin{array}{c}\text { Temp } \\
\left({ }^{\circ} \mathrm{C}\right)\end{array}$ & $\begin{array}{c}\text { Intial } \\
\text { Hydraulic } \\
\text { Gradient }\end{array}$ & $\begin{array}{c}\text { Final } \\
\text { Hydraulic } \\
\text { Gradient }\end{array}$ & $\begin{array}{c}\mathrm{k} \\
\mathrm{cm} / \mathrm{sec}\end{array}$ & $\begin{array}{c}\mathrm{k} \\
\mathrm{cm} / \mathrm{sec} \\
\mathrm{at} 20{ }^{\circ} \mathrm{C}\end{array}$ \\
\hline 5820 & 1.50 & 26.00 & 25.10 & 0.90 & 22.8 & 22.4 & 21.5 & $4.72 \mathrm{E}-09$ & $4.41 \mathrm{E}-09$ \\
\hline 12810 & 1.50 & 26.00 & 24.80 & 1.20 & 22.9 & 22.4 & 21.3 & $2.88 \mathrm{E}-09$ & $2.68 \mathrm{E}-09$ \\
\hline 19920 & 1.50 & 26.00 & 24.50 & 1.50 & 22.9 & 22.4 & 21.0 & $2.33 \mathrm{E}-09$ & $2.17 \mathrm{E}-09$ \\
\hline 5040 & 1.50 & 25.80 & 25.30 & 0.50 & 22.8 & 22.2 & 21.7 & $3.02 \mathrm{E}-09$ & $2.83 \mathrm{E}-09$ \\
\hline 7380 & 1.50 & 27.60 & 25.90 & 1.70 & 22.3 & 23.9 & 22.2 & $6.70 \mathrm{E}-09$ & $6.34 \mathrm{E}-09$ \\
\hline 14700 & 1.50 & 27.60 & 24.60 & 3.00 & 22.9 & 23.9 & 21.0 & $6.10 \mathrm{E}-09$ & $5.70 \mathrm{E}-09$ \\
\hline 21840 & 1.50 & 27.60 & 23.40 & 4.20 & 22.6 & 23.9 & 19.9 & $5.91 \mathrm{E}-09$ & $5.55 \mathrm{E}-09$ \\
\hline
\end{tabular}

\begin{tabular}{|c|c|c|c|c|}
\hline No. of Trials & Sample & Max. Density & Compaction & $\begin{array}{c}\text { Sample } \\
\text { Orientation }\end{array}$ \\
\hline 7 & Core & N/A & N/A & Vertical \\
\hline
\end{tabular}

$A=46.90 \mathrm{~cm}^{2}$

$\mathrm{L}=\quad 13.75 \mathrm{~cm}$

$\mathrm{S}=\mathrm{L} / \mathrm{A}=0.29316 \mathrm{l} / \mathrm{cm}$

$\begin{aligned} \mathrm{a}_{\mathrm{p}}= & 0.031416 \mathrm{~cm}^{2} \\ \mathrm{M}_{1}= & 0.03018 \\ \mathrm{M}_{2}= & 1.04095 \\ \mathrm{C}=\mathrm{M}_{1} \mathrm{~S} /\left(\mathrm{G}_{\mathrm{Hg}}-1\right)= & 0.0007039 \text { for } 15^{\circ} \text { to } 25^{\circ}\end{aligned}$

Avg. $\mathrm{k}$ at $20^{\circ} \mathrm{C} \quad 4.2 \mathrm{E}-09 \mathrm{~cm} / \mathrm{sec}$

Remarks: 


\section{amec $^{\theta}$}

\section{HYDRAULIC CONDUCTIVITY}

$\begin{array}{llll}\text { Project No. } & \mathbf{6 1 5 5 - 0 8 - 0 0 3 1 . 3 4} & \text { Tested By } & \text { JEL } \\ \text { Project Name } & \text { SDU-6 Task3 Grout Samples } & \text { Test Date } & 7 / 10 / 2012 \\ \text { Boring No. } & \text { V0.5-3 } & \text { Reviewed By } & \text { JW } 2 G h \\ \text { Sample No. } & \text { V0.5-3 } & \text { Review Date } & 8 / 1 / 2012 \\ \text { Sample Depth } & \text { N/A } & \text { Lab No. } & 11619\end{array}$

Sample Description Grout Core

ASTM D5084 - Method F (CVFH)

\begin{tabular}{|l|l|}
\hline Sample Type: & Core \\
\hline Sample Orientation: & Vertical \\
\hline Initial Water Content, \%: & 44.1 \\
\hline Wet Unit Weight, pcf: & 107.6 \\
\hline Dry Unit Weight, pcf: & 74.7 \\
\hline Compaction, \%: & N/A \\
\hline Hydraulic Conductivity, cm/sec. @20 ${ }^{\circ} \mathrm{C}$ & $\mathbf{3 . 1 E - 0 9}$ \\
\hline
\end{tabular}

Remarks: 
SRNL-STI-2012-00522

Revision 0

PERMEABILITY TEST

(ASTM D5084 - 03) (Method F, Constant Volume Falling Head)

Project Number 6155-08-0031.34

Boring No. V0.5-3 Reviewed By JW Q\& f

Sample No. V0.5-3 Review Date 08/01/12

Sample Depth N/A Lab No. 11619

Sample Description Grout Core

\begin{tabular}{|c|c|c|c|c|c|}
\hline \multicolumn{4}{|c|}{ Initial Sample Data } & \multicolumn{2}{|c|}{ Final Sample Data } \\
\hline \multicolumn{2}{|c|}{ Length, in } & \multicolumn{2}{|l|}{ Diameter, in } & Pan No. & N/A \\
\hline Location 1 & 5.501 & Location 1 & 3.006 & Wet Soil+Pan, grams & 1108.23 \\
\hline Location 2 & 5.496 & Location 2 & 3.003 & Dry Soil + Pan, grams & 769.05 \\
\hline Location 3 & 5.491 & Location 3 & 3.034 & Pan Weight, grams & \\
\hline Average & 5.496 & Average & 3.014 & Moisture Content, \% & 44.1 \\
\hline Volume, in ${ }^{3}$ & 39.22 & Wet Soil + Tare, grams & 1107.89 & Dry Unit Weight, pcf & 74.7 \\
\hline SG Assumed & 2.40 & Tare Weight, grams & 0.00 & Saturation, \% & 105.3 \\
\hline Soil Sample Wt., g & 1107.89 & Dry Soil +Tare, grams & 769.05 & Diameter, in. & $\mathrm{N} / \mathrm{A}$ \\
\hline Dry UW, pef & 74.7 & Moisture Content, \% & 44.1 & Length, in. & N/A \\
\hline Saturation, \% & 105.2 & & & Volume, in ${ }^{3}$ & N/A \\
\hline
\end{tabular}

amec 0

\begin{tabular}{|r|r|r|r|r|r|r|r|r|r|}
\hline $\begin{array}{c}\text { Elapsed Time } \\
(\mathrm{sec})\end{array}$ & $\begin{array}{c}\mathrm{z}_{\mathrm{o}} \\
(\mathrm{cm})\end{array}$ & $\begin{array}{c}\mathrm{za} \\
(\mathrm{cm})\end{array}$ & $\begin{array}{c}\mathrm{zb} \\
(\mathrm{cm})\end{array}$ & $\begin{array}{c}\Delta \mathrm{z}_{\mathrm{p}} \\
(\mathrm{cm})\end{array}$ & $\begin{array}{c}\text { Temp } \\
\left({ }^{\circ} \mathrm{C}\right)\end{array}$ & $\begin{array}{c}\text { Intial } \\
\text { Hydraulic } \\
\text { Gradient }\end{array}$ & $\begin{array}{c}\text { Final } \\
\text { Hydraulic } \\
\text { Gradient }\end{array}$ & $\begin{array}{c}\mathrm{k} \\
\mathrm{cm} / \mathrm{sec}\end{array}$ & $\begin{array}{c}\mathrm{k} \\
\mathrm{cm} / \mathrm{sec} \\
\mathrm{at} 20^{\circ} \mathrm{C}\end{array}$ \\
\hline 60570 & 1.60 & 27.30 & 22.15 & 5.15 & 22.6 & 23.1 & 18.3 & $2.81 \mathrm{E}-09$ & $2.64 \mathrm{E}-09$ \\
\hline 5400 & 1.60 & 27.10 & 26.10 & 1.00 & 22.8 & 23.0 & 22.0 & $5.62 \mathrm{E}-09$ & $5.26 \mathrm{E}-09$ \\
\hline 12240 & 1.60 & 27.10 & 25.50 & 1.60 & 22.9 & 23.0 & 21.5 & $4.02 \mathrm{E}-09$ & $3.75 \mathrm{E}-09$ \\
\hline 19380 & 1.60 & 27.10 & 24.90 & 2.20 & 22.9 & 23.0 & 20.9 & $3.53 \mathrm{E}-09$ & $3.30 \mathrm{E}-09$ \\
\hline 25080 & 1.60 & 27.10 & 24.60 & 2.50 & 23.0 & 23.0 & 20.6 & $3.12 \mathrm{E}-09$ & $2.91 \mathrm{E}-09$ \\
\hline 82560 & 1.60 & 27.10 & 22.00 & 5.10 & 22.5 & 23.0 & 18.2 & $2.06 \mathrm{E}-09$ & $1.94 \mathrm{E}-09$ \\
\hline 4980 & 1.60 & 25.70 & 25.40 & 0.30 & 22.8 & 21.7 & 21.4 & $1.91 \mathrm{E}-09$ & $1.78 \mathrm{E}-09$ \\
\hline
\end{tabular}

$\begin{aligned} & \begin{array}{cc}\text { No. of Trials } & \begin{array}{c}\text { Samp } \\ \text { Type }\end{array} \\ \hline 7 & \text { Core } \\ \mathrm{a}_{2}= & 0.76712 \mathrm{~cm}^{2} \\ \mathrm{~A} & 46.04 \mathrm{~cm}^{2} \\ \mathrm{~L} & =\quad 13.96 \mathrm{~cm} \\ \mathrm{~S}=\mathrm{L} / \mathrm{A}= & 0.303211 / \mathrm{cm}\end{array}\end{aligned}$

Sample Max. Density Compaction Sample

Type $\quad(\mathrm{pc})$

$\% \quad$ Orientation

Avg. $\mathrm{k}$ at $20^{\circ} \mathrm{C} \quad 3.1 \mathrm{E}-09 \mathrm{~cm} / \mathrm{sec}$

$\begin{aligned} \mathrm{a}_{\mathrm{p}}= & 0.031416 \mathrm{~cm}^{2} \\ \mathrm{M}_{1}= & 0.03018 \\ \mathrm{M}_{2}= & 1.04095 \\ \mathrm{C}=\mathrm{M}_{1} \mathrm{~S} /\left(\mathrm{G}_{\mathrm{Hg}}-1\right)= & 0.0007280 \text { for } 15^{\circ} \text { to } 25^{\circ}\end{aligned}$

Remarks

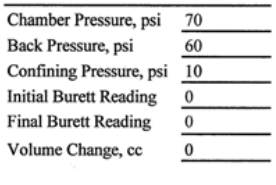

Permeant used water 


\section{amec $^{\theta}$}

\section{HYDRAULIC CONDUCTIVITY}

$\begin{array}{llll}\text { Project No. } & \text { 6155-08-0031.34 } & \text { Tested By } & \text { JEL } \\ \text { Project Name } & \text { SDU-6 Task 3 Grout Samples } & \text { Test Date } & 7 / 10 / 2012 \\ \text { Boring No. } & \text { V1.0-1 } & \text { Reviewed By } J W \delta \varepsilon \gamma \\ \text { Sample No. } & \text { V1.0-1 } & \text { Review Date } & 8 / 1 / 2012 \\ \text { Sample Depth } & \text { N/A } & \text { Lab No. } & 11620\end{array}$

Sample Description Grout Core

ASTM D5084 - Method F (CVFH)

\begin{tabular}{|l|l|}
\hline Sample Type: & Core \\
\hline Sample Orientation: & Vertical \\
\hline Initial Water Content, \%: & 43.8 \\
\hline Wet Unit Weight, pcf: & 107.6 \\
\hline Dry Unit Weight, pcf: & 74.8 \\
\hline Compaction, \%: & N/A \\
\hline Hydraulic Conductivity, cm/sec. @20 ${ }^{\circ} \mathrm{C}$ & $2.5 \mathrm{E}-09$ \\
\hline
\end{tabular}

Remarks: 
SRNL-STI-2012-00522

Revision 0

PERMEABILITY TEST

(ASTM D5084 - 03) (Method F, Constant Volume Falling Head)

Project Number 6155-08-0031.34 Tested By JEL

Project Name SDU-6 Task 3 Grout Samples Test Date 07/10/12

Boring No. V1.0-1 Reviewed By JW g्द

Sample No. V1.0-1 Review Date 08/01/12

Sample Depth N/A Lab No. 11620

Sample Description Grout Core

\begin{tabular}{|c|r|c|r|c|r|r|}
\hline \multicolumn{4}{|c|}{ Initial Sample Data } & \multicolumn{3}{c|}{ Final Sample Data } \\
\hline Length, in & \multicolumn{2}{|c|}{ Diameter, in } & Pan No. & N/A \\
\hline Location 1 & 4.966 & Location 1 & 3.000 & Wet Soil+Pan, grams & 993.57 \\
\hline Location 2 & 4.975 & Location 2 & 3.008 & Dry Soil + Pan, grams & 690.68 \\
\hline Location3 & 4.960 & Location 3 & 3.001 & Pan Weight, grams & 0 \\
\hline Average & 4.967 & Average & 3.003 & Moisture Content, \% & 43.9 \\
\hline Volume, in & 3 & 35.18 & Wet Soil + Tare, grams & 993.31 & Dry Unit Weight, pcf & 74.8 \\
\hline SG Assumed & 2.40 & Tare Weight, grams & 0.00 & Saturation, \% & 105.0 \\
\hline Soil Sample Wt., g & 993.31 & Dry Soil +Tare, grams & 690.68 & Diameter, in. & N/A \\
\hline Dry UW, pcf & 74.8 & Moisture Content, \% & 43.8 & Length, in. & N/A \\
\hline Saturation, \% & 104.9 & & & Volume, in ${ }^{3}$ & N/A \\
\hline
\end{tabular}

\begin{tabular}{|r|c|c|c|c|c|c|c|c|c|}
\hline $\begin{array}{c}\text { Elapsed Time } \\
(\mathrm{sec})\end{array}$ & $\begin{array}{c}\mathrm{z}_{\mathrm{o}} \\
(\mathrm{cm})\end{array}$ & $\begin{array}{c}\mathrm{za} \\
(\mathrm{cm})\end{array}$ & $\begin{array}{c}\mathrm{zb} \\
(\mathrm{cm})\end{array}$ & $\begin{array}{c}\Delta \mathrm{z}_{\mathrm{p}} \\
(\mathrm{cm})\end{array}$ & $\begin{array}{c}\text { Temp } \\
\left({ }^{\circ} \mathrm{C}\right)\end{array}$ & $\begin{array}{c}\text { Intial } \\
\text { Hydraulic } \\
\text { Gradient }\end{array}$ & $\begin{array}{c}\text { Final } \\
\text { Hydraulic } \\
\text { Gradient }\end{array}$ & $\begin{array}{c}\mathrm{k} \\
\mathrm{cm} / \mathrm{sec}\end{array}$ & $\begin{array}{c}\mathrm{k} \\
\mathrm{cm} / \mathrm{sec} \\
\mathrm{at} 20^{\circ} \mathrm{C}\end{array}$ \\
\hline 60780 & 1.70 & 27.70 & 20.65 & 7.05 & 22.6 & 25.9 & 18.6 & $3.62 \mathrm{E}-09$ & $3.40 \mathrm{E}-09$ \\
\hline 6600 & 1.70 & 26.60 & 25.90 & 0.70 & 22.8 & 24.8 & 24.1 & $2.98 \mathrm{E}-09$ & $2.79 \mathrm{E}-09$ \\
\hline 13560 & 1.70 & 26.60 & 25.20 & 1.40 & 22.9 & 24.8 & 23.4 & $2.95 \mathrm{E}-09$ & $2.75 \mathrm{E}-09$ \\
\hline 20640 & 1.70 & 26.60 & 24.70 & 1.90 & 22.9 & 24.8 & 22.8 & $2.66 \mathrm{E}-09$ & $2.48 \mathrm{E}-09$ \\
\hline 26400 & 1.70 & 26.60 & 24.30 & 2.30 & 23.0 & 24.8 & 22.4 & $2.54 \mathrm{E}-09$ & $2.36 \mathrm{E}-09$ \\
\hline 83940 & 1.70 & 26.50 & 21.90 & 4.60 & 22.5 & 24.7 & 19.9 & $1.69 \mathrm{E}-09$ & $1.60 \mathrm{E}-09$ \\
\hline 5040 & 1.70 & 25.20 & 24.80 & 0.40 & 22.8 & 23.4 & 23.0 & $2.35 \mathrm{E}-09$ & $2.20 \mathrm{E}-09$ \\
\hline
\end{tabular}

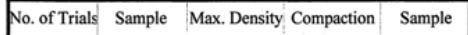

\begin{tabular}{|c|c|c|c|c|}
\hline No. of Trials & $\begin{array}{c}\text { Sample } \\
\text { Type }\end{array}$ & $\begin{array}{c}\text { Max. Density } \\
(\mathrm{pcf})\end{array}$ & $\begin{array}{c}\text { Compaction } \\
\%\end{array}$ & $\begin{array}{c}\text { Sample } \\
\text { Orientation } \\
\end{array}$ \\
\hline 7 & Core & N/A & $\mathrm{N} /$ & \\
\hline
\end{tabular}

Avg. $\mathrm{k}$ at $20^{\circ} \mathrm{C} \quad \underline{2.5 \mathrm{E}-09} \mathrm{~cm} / \mathrm{sec}$

$$
\begin{aligned}
& \mathrm{a}_{\mathrm{a}}=\quad 0.76712 \mathrm{~cm}^{2} \\
& \mathrm{~A}=\quad 45.69 \mathrm{~cm}^{2} \\
& \mathrm{~L}=\quad 12.62 \mathrm{~cm}
\end{aligned}
$$

$\mathrm{S}=\mathrm{L} / \mathrm{A}=0.27610 \mathrm{1} / \mathrm{cm}$

$\mathrm{a}_{\mathrm{p}}=0.031416 \mathrm{~cm}^{2}$

$\mathrm{M}_{1}=\quad 0.03018$

$\mathrm{M}_{2}=1.04095$

$\mathrm{C}=\mathrm{M}_{1} \mathrm{~S} /\left(\mathrm{G}_{\mathrm{H}_{8}}-1\right)=0.0006629$ for $15^{\circ}$ to $25^{\circ}$

$\operatorname{amec}^{\theta}$

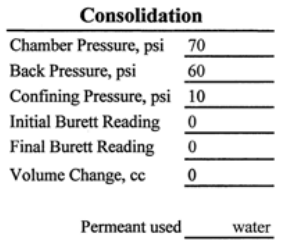




\section{amec $^{\theta}$}

\section{HYDRAULIC CONDUCTIVITY}

$\begin{array}{llll}\text { Project No. } & \text { 6155-08-0031.34 } & \text { Tested By } & \text { JEL } \\ \text { Project Name } & \text { SDU-6 Task 3 Grout Samples } & \text { Test Date } & 7 / 17 / 2012 \\ \text { Boring No. } & \text { V1.0-2 } & \text { Reviewed By } & \text { JW } \\ \text { Sample No. } & V 1.0-2 & \text { Review Date } & 8 / 1 / 2012 \\ \text { Sample Depth } & N / A & \text { Lab No. } & 11621\end{array}$

Sample Description Grout Core

ASTM D5084 - Method F (CVFH)

\begin{tabular}{|l|l|}
\hline Sample Type: & Core \\
\hline Sample Orientation: & Vertical \\
\hline Initial Water Content, \%: & 42.7 \\
\hline Wet Unit Weight, pcf: & 106.1 \\
\hline Dry Unit Weight, pcf: & 74.4 \\
\hline Compaction, \%: & N/A \\
\hline Hydraulic Conductivity, cm/sec. @20 C & $2.2 E-09$ \\
\hline
\end{tabular}

Remarks: 
SRNL-STI-2012-00522

Revision 0

PERMEABILITY TEST

(ASTM D5084 - 03) (Method F, Constant Volume Falling Head)

Project Number 6155-08-0031.34

Project Name SDU-6 Task 3 Grout Samples Test Date 07/17/12

Boring No. V1.0-2 Reviewed By JW Яद

Sample No. V1.0-2 Review Date 08/01/12

Sample Depth N/A Lab No. 1162

Sample Description Grout Core

\begin{tabular}{|c|r|c|r|c|r|r|}
\hline \multicolumn{4}{|c|}{ Initial Sample Data } & \multicolumn{2}{c|}{ Final Sample Data } \\
\hline \multicolumn{2}{|c|}{ Length, in } & \multicolumn{2}{|c|}{ Diameter, in } & Pan No. & N/A \\
\hline Location 1 & 5.078 & Location 1 & 3.028 & Wet Soil+Pan, grams & 1014.02 \\
\hline Location 2 & 5.072 & Location 2 & 3.039 & Dry Soil + Pan, grams & 710.53 \\
\hline Location3 & 5.030 & Location 3 & 3.013 & Pan Weight, grams & 0 \\
\hline Average & 5.060 & Average & 3.027 & Moisture Content, \% & 42.7 \\
\hline Volume, in & 3 & 36.41 & Wet Soil + Tare, grams & 1013.74 & Dry Unit Weight, pcf & 74.4 \\
\hline SG Assumed & 2.40 & Tare Weight, grams & 0.00 & Saturation, \% & 101.1 \\
\hline Soil Sample Wt., g & 1013.74 & Dry Soil +Tare, grams & 710.53 & Diameter, in. & N/A \\
\hline Dry UW, pcf & 74.4 & Moisture Content, \% & 42.7 & Length, in. & N/A \\
\hline Saturation, \% & 101.0 & & & Volume, in & N/A \\
\hline
\end{tabular}

ameco

\begin{tabular}{|r|c|c|c|c|c|c|c|c|c|}
\hline $\begin{array}{c}\text { Elapsed Time } \\
(\mathrm{sec})\end{array}$ & $\begin{array}{c}\mathrm{z}_{\mathrm{o}} \\
(\mathrm{cm})\end{array}$ & $\begin{array}{c}\mathrm{za} \\
(\mathrm{cm})\end{array}$ & $\begin{array}{c}\mathrm{zb} \\
(\mathrm{cm})\end{array}$ & $\begin{array}{c}\Delta \mathrm{z}_{\mathrm{p}} \\
(\mathrm{cm})\end{array}$ & $\begin{array}{c}\text { Temp } \\
\left({ }^{\circ} \mathrm{C}\right)\end{array}$ & $\begin{array}{c}\text { Intial } \\
\text { Hydraulic } \\
\text { Gradient }\end{array}$ & $\begin{array}{c}\text { Final } \\
\text { Hydraulic } \\
\text { Gradient }\end{array}$ & $\begin{array}{c}\mathrm{k} \\
\mathrm{cm} / \mathrm{sec}\end{array}$ & $\begin{array}{c}\mathrm{k} \\
\mathrm{cm} / \mathrm{sec} \\
\mathrm{at} \mathrm{20}{ }^{\circ} \mathrm{C}\end{array}$ \\
\hline 1050 & 2.20 & 24.80 & 24.60 & 0.20 & 21.9 & 22.1 & 21.9 & $5.86 \mathrm{E}-09$ & $5.60 \mathrm{E}-09$ \\
\hline 4080 & 2.20 & 24.80 & 24.50 & 0.30 & 22.0 & 22.1 & 21.8 & $2.27 \mathrm{E}-09$ & $2.16 \mathrm{E}-09$ \\
\hline 9240 & 2.20 & 24.80 & 24.40 & 0.40 & 22.4 & 22.1 & 21.7 & $1.34 \mathrm{E}-09$ & $1.26 \mathrm{E}-09$ \\
\hline 5340 & 2.20 & 27.00 & 26.70 & 0.30 & 22.8 & 24.3 & 23.9 & $1.58 \mathrm{E}-09$ & $1.48 \mathrm{E}-09$ \\
\hline 10800 & 2.20 & 27.00 & 26.55 & 0.45 & 22.6 & 24.3 & 23.8 & $1.17 \mathrm{E}-09$ & $1.10 \mathrm{E}-09$ \\
\hline 3540 & 2.20 & 27.10 & 26.80 & 0.30 & 22.6 & 24.4 & 24.0 & $2.37 \mathrm{E}-09$ & $2.23 \mathrm{E}-09$ \\
\hline 61200 & 2.20 & 27.10 & 24.30 & 2.80 & 21.9 & 24.4 & 21.5 & $1.35 \mathrm{E}-09$ & $1.29 \mathrm{E}-09$ \\
\hline
\end{tabular}

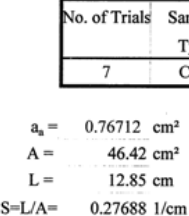

\begin{tabular}{cc|c|c|}
$\begin{array}{c}\text { Sample } \\
\text { Type }\end{array}$ & $\begin{array}{c}\text { Max. Density Compaction } \\
\text { (pef) }\end{array}$ & $\begin{array}{c}\text { Sample } \\
\text { Orientation }\end{array}$ \\
\hline
\end{tabular}

\begin{tabular}{c|c}
$\%$ & Orientation \\
\hline N/A & Vertical \\
\hline
\end{tabular}

Avg. $\mathrm{k}$ at $20^{\circ} \mathrm{C} \quad \underline{2.2 \mathrm{E}-09} \mathrm{~cm} / \mathrm{sec}$

Core N/A

$a_{\mathrm{p}}=0.031416 \mathrm{~cm}^{2}$

$\mathrm{L}=\quad 12.85 \mathrm{~cm}$

$\mathrm{M}_{1}=0.03018$

$\mathrm{M}_{2}=1.04095$

$\mathrm{S}=\mathrm{L} / \mathrm{A}=0.27688 \mathrm{1} / \mathrm{cm}$

$\mathrm{C}=\mathrm{M}_{1} \mathrm{~S} /\left(\mathrm{G}_{\mathrm{Hg}_{\mathrm{g}}}-1\right)=0.0006648$ for $15^{\circ}$ to $25^{\circ}$

Remarks:

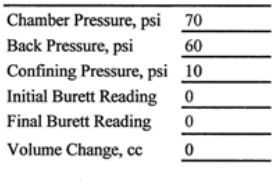




\section{amec $^{\theta}$}

\section{HYDRAULIC CONDUCTIVITY}

$\begin{array}{llll}\text { Project No. } & \text { 6155-08-0031.34 } & \text { Tested By } & \text { JEL } \\ \text { Project Name } & \text { SDU-6 Task 3 Grout Samples } & \text { Test Date } & 7 / 17 / 2012 \\ \text { Boring No. } & V 1.0-3 & \text { Reviewed By } & J W f \& \mathcal{H} \\ \text { Sample No. } & V 1.0-3 & \text { Review Date } & 8 / 1 / 2012 \\ \text { Sample Depth } & \text { N/A } & \text { Lab No. } & 11622\end{array}$

Sample Description Grout Core

ASTM D5084 - Method F (CVFH)

\begin{tabular}{|l|l|}
\hline Sample Type: & Core \\
\hline Sample Orientation: & Vertical \\
\hline Initial Water Content, \%: & 43.5 \\
\hline Wet Unit Weight, pcf: & 107.5 \\
\hline Dry Unit Weight, pcf: & 74.9 \\
\hline Compaction, \%: & N/A \\
\hline Hydraulic Conductivity, cm/sec. @20 C & $4.0 E-09$ \\
\hline
\end{tabular}

Remarks: 
SRNL-STI-2012-00522

Revision 0

PERMEABILITY TEST

(ASTM D5084 - 03) (Method F, Constant Volume Falling Head)

amec $^{\theta}$

Project Number 6155-08-0031.34

Tested By JEL

Project Name SDU-6 Task 3 Grout Samples Test Date 07/17/12

Boring No. V1.0-3 Reviewed By JW GA I

Sample No. V1.0-3 Review Date 08/01/12

Sample Depth N/A Lab No. 11622

Sample Description $\quad$ Grout Core

\begin{tabular}{|c|r|c|r|c|r|r|}
\hline \multicolumn{3}{|c|}{ Initial Sample Data } & \multicolumn{3}{c|}{ Final Sample Data } \\
\hline Length, in & \multicolumn{2}{|c|}{ Diameter, in } & Pan No. & N/A \\
\hline Location 1 & 5.263 & Location 1 & 3.029 & Wet Soil+Pan, grams & 1063.47 \\
\hline Location 2 & 5.318 & Location 2 & 3.009 & Dry Soil + Pan, grams & 740.65 \\
\hline Location3 & 5.301 & Location 3 & 2.994 & Pan Weight, grams & 0 \\
\hline Average & 5.294 & Average & 3.011 & Moisture Content, \% & 43.6 \\
\hline Volume, in & 37 & 37.69 & Wet Soil + Tare, grams & 1063.08 & Dry Unit Weight, pcf & 74.9 \\
\hline SG Assumed & 2.40 & Tare Weight, grams & 0.00 & Saturation, \% & 104.6 \\
\hline Soil Sample Wt., g & 1063.08 & Dry Soil +Tare, grams & 740.65 & Diameter, in. & N/A \\
\hline Dry UW, pcf & 74.9 & Moisture Content, \% & 43.5 & Length, in. & N/A \\
\hline Saturation, \% & 104.4 & & & Volume, in & N/A \\
\hline
\end{tabular}

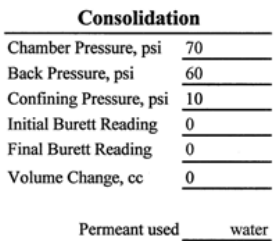

\begin{tabular}{|r|c|c|c|c|c|c|c|c|c|}
\hline $\begin{array}{c}\text { Elapsed Time } \\
(\mathrm{sec})\end{array}$ & $\begin{array}{c}\mathrm{z}_{\mathrm{o}} \\
(\mathrm{cm})\end{array}$ & $\begin{array}{c}\mathrm{za} \\
(\mathrm{cm})\end{array}$ & $\begin{array}{c}\mathrm{zb} \\
(\mathrm{cm})\end{array}$ & $\begin{array}{c}\Delta \mathrm{z}_{\mathrm{p}} \\
(\mathrm{cm})\end{array}$ & $\begin{array}{c}\text { Temp } \\
\left({ }^{\circ} \mathrm{C}\right)\end{array}$ & $\begin{array}{c}\text { Intial } \\
\text { Hydraulic } \\
\text { Gradient }\end{array}$ & $\begin{array}{c}\text { Final } \\
\text { Hydraulic } \\
\text { Gradient }\end{array}$ & $\begin{array}{c}\mathrm{k} \\
\mathrm{cm} / \mathrm{sec}\end{array}$ & $\begin{array}{c}\mathrm{k} \\
\mathrm{cm} / \mathrm{sec} \\
\mathrm{at} 20{ }^{\circ} \mathrm{C}\end{array}$ \\
\hline 900 & 1.70 & 24.40 & 24.25 & 0.15 & 21.9 & 21.2 & 21.1 & $5.39 \mathrm{E}-09$ & $5.15 \mathrm{E}-09$ \\
\hline 2850 & 1.70 & 24.40 & 23.90 & 0.50 & 22.0 & 21.2 & 20.7 & $5.72 \mathrm{E}-09$ & $5.45 \mathrm{E}-09$ \\
\hline 5100 & 1.70 & 24.40 & 23.60 & 0.80 & 22.0 & 21.2 & 20.4 & $5.15 \mathrm{E}-09$ & $4.91 \mathrm{E}-09$ \\
\hline 10320 & 1.70 & 24.40 & 23.20 & 1.20 & 22.4 & 21.2 & 20.1 & $3.86 \mathrm{E}-09$ & $3.64 \mathrm{E}-09$ \\
\hline 16020 & 1.70 & 24.40 & 22.80 & 1.60 & 22.8 & 21.2 & 19.7 & $3.34 \mathrm{E}-09$ & $3.13 \mathrm{E}-09$ \\
\hline 21480 & 1.70 & 24.40 & 22.50 & 1.90 & 22.6 & 21.2 & 19.4 & $2.98 \mathrm{E}-09$ & $2.80 \mathrm{E}-09$ \\
\hline 24420 & 1.70 & 24.40 & 22.30 & 2.10 & 22.8 & 21.2 & 19.2 & $2.91 \mathrm{E}-09$ & $2.73 \mathrm{E}-09$ \\
\hline
\end{tabular}

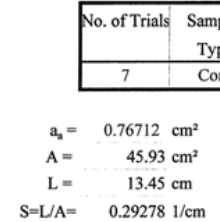

\begin{tabular}{c|c|c|c|}
\hline $\begin{array}{c}\text { Sample } \\
\text { Type }\end{array}$ & $\begin{array}{c}\text { Max. Density Compaction } \\
\text { (pcf) }\end{array}$ & $\begin{array}{c}\text { Sample } \\
\text { Orientation }\end{array}$ \\
\hline
\end{tabular}

\begin{tabular}{c|c|}
$\%$ & Orientation \\
\hline N/A & Vertical \\
\hline
\end{tabular}

Avg. $\mathrm{k}$ at $20^{\circ} \mathrm{C} \quad \underline{4.0 \mathrm{E}-09} \mathrm{~cm} / \mathrm{sec}$

/ $=0.292781 / \mathrm{cm}$

$\mathrm{a}_{\mathrm{p}}=0.031416 \mathrm{~cm}^{2}$

$\mathrm{M}_{1}=0.03018$

$\mathrm{M}_{2}=1.04095$

$\mathrm{C}=\mathrm{M}_{1} \mathrm{~S} /\left(\mathrm{G}_{\mathrm{Hg}}-1\right)=0.0007029$ for $15^{\circ}$ to $25^{\circ}$

Remarks 


\section{amec $^{\theta}$}

\section{HYDRAULIC CONDUCTIVITY}

\begin{tabular}{|c|c|c|c|}
\hline Project No. & $6155-08-0031.34$ & Tested By & $J E L$ \\
\hline Project Name & SDU-6 Task 3 Grout Samples & Test Date & $7 / 17 / 2012$ \\
\hline Boring No. & H0.5-1 & Reviewed By & $J W \emptyset \varepsilon \gamma$ \\
\hline Sample No. & H0.5-1 & Review Date & $8 / 1 / 2012$ \\
\hline Sample Depth & $N / A$ & Lab No. & 11623 \\
\hline
\end{tabular}

Sample Description Grout Core

ASTM D5084 - Method F (CVFH)

\begin{tabular}{|l|l|}
\hline Sample Type: & Core \\
\hline Sample Orientation: & Vertical \\
\hline Initial Water Content, \%: & 43.6 \\
\hline Wet Unit Weight, pcf: & 107.4 \\
\hline Dry Unit Weight, pcf: & 74.8 \\
\hline Compaction, \%: & N/A \\
\hline Hydraulic Conductivity, cm/sec. @20 C & $3.6 E-08$ \\
\hline
\end{tabular}

Remarks: 
SRNL-STI-2012-00522

Revision 0

PERMEABILITY TEST

(ASTM D5084 - 03) (Method F, Constant Volume Falling Head)

amec 0

Project Number 6155-08-0031.34

Tested By JEL

Project Name SDU-6 Task 3 Grout Samples Test Date 07/17/12

Boring No. H0.5-1 Reviewed By JW g\&f

Sample No. H0.5-1 Review Date 08/01/12

Sample Depth N/A Lab No. 11623

Sample Description Grout Core

\begin{tabular}{|c|r|c|r|c|c|r|}
\hline \multicolumn{4}{|c|}{ Initial Sample Data } & \multicolumn{3}{c|}{ Final Sample Data } \\
\hline Length, in & \multicolumn{2}{|c|}{ Diameter, in } & Pan No. & N/A \\
\hline Location 1 & 4.398 & Location 1 & 2.976 & Wet Soil+Pan, grams & 882.07 \\
\hline Location 2 & 4.463 & Location 2 & 2.996 & Dry Soil + Pan, grams & 614.31 \\
\hline Location3 & 4.510 & Location 3 & 2.996 & Pan Weight, grams & 0 \\
\hline Average & 4.457 & Average & 2.989 & Moisture Content, \% & 43.6 \\
\hline Volume, in & 31.28 & Wet Soil + Tare, grams & 882.05 & Dry Unit Weight, pcf & 74.8 \\
\hline SG Assumed & 2.40 & Tare Weight, grams & 0.00 & Saturation, \% & 104.4 \\
\hline Soil Sample Wt., g & 882.05 & Dry Soil + Tare, grams & 614.31 & Diameter, in. & N/A \\
\hline Dry UW, pcf & 74.8 & Moisture Content, \% & 43.6 & Length, in. & N/A \\
\hline Saturation, \% & 104.4 & & & Volume, in & N/A \\
\hline
\end{tabular}

Consolidation

Chamber Pressure, psi 70

Back Pressure, psi

Confining Pressure, psi

Initial Burett Reading

Final Burett Reading

Final Burett Reading

Volume Change, cc

Permeant used water

\begin{tabular}{|r|r|r|r|r|r|r|r|r|r|}
\hline $\begin{array}{c}\text { Elapsed Time } \\
(\mathrm{sec})\end{array}$ & $\begin{array}{c}\mathrm{z}_{\mathrm{o}} \\
(\mathrm{cm})\end{array}$ & $\begin{array}{c}\mathrm{za} \\
(\mathrm{cm})\end{array}$ & $\begin{array}{c}\mathrm{zb} \\
(\mathrm{cm})\end{array}$ & $\begin{array}{c}\Delta \mathrm{z}_{\mathrm{p}} \\
(\mathrm{cm})\end{array}$ & $\begin{array}{c}\text { Temp } \\
\left({ }^{\circ} \mathrm{C}\right)\end{array}$ & $\begin{array}{c}\text { Intial } \\
\text { Hydraulic } \\
\text { Gradient }\end{array}$ & $\begin{array}{c}\text { Final } \\
\text { Hydraulic } \\
\text { Gradient }\end{array}$ & $\begin{array}{c}\mathrm{k} \\
\mathrm{cm} / \mathrm{sec}\end{array}$ & $\begin{array}{c}\mathrm{k} \\
\mathrm{cm} / \mathrm{sec} \\
\mathrm{at} 20^{\circ} \mathrm{C}\end{array}$ \\
\hline 1725 & 1.70 & 26.00 & 23.20 & 2.80 & 21.9 & 27.0 & 23.7 & $4.45 \mathrm{E}-08$ & $4.25 \mathrm{E}-08$ \\
\hline 2220 & 1.70 & 26.00 & 22.70 & 3.30 & 21.9 & 27.0 & 23.2 & $4.12 \mathrm{E}-08$ & $3.94 \mathrm{E}-08$ \\
\hline 2850 & 1.70 & 26.00 & 22.20 & 3.80 & 21.9 & 27.0 & 22.6 & $3.74 \mathrm{E}-08$ & $3.58 \mathrm{E}-08$ \\
\hline 3510 & 1.70 & 26.00 & 21.60 & 4.40 & 22.0 & 27.0 & 21.9 & $3.57 \mathrm{E}-08$ & $3.40 \mathrm{E}-08$ \\
\hline 930 & 1.70 & 23.80 & 22.75 & 1.05 & 22.0 & 24.5 & 23.3 & $3.27 \mathrm{E}-08$ & $3.12 \mathrm{E}-08$ \\
\hline 1620 & 1.70 & 23.80 & 21.70 & 2.10 & 22.0 & 24.5 & 22.1 & $3.86 \mathrm{E}-08$ & $3.68 \mathrm{E}-08$ \\
\hline 2280 & 1.70 & 23.80 & 21.30 & 2.50 & 22.0 & 24.5 & 21.6 & $3.30 \mathrm{E}-08$ & $3.14 \mathrm{E}-08$ \\
\hline
\end{tabular}

\begin{tabular}{|c|c|c|c|c|}
\hline No. of Trials & $\begin{array}{c}\text { Sample } \\
\text { Type }\end{array}$ & $\begin{array}{c}\text { Max. Density } \\
\text { (pcf) }\end{array}$ & $\begin{array}{c}\text { Compaction } \\
\%\end{array}$ & $\begin{array}{c}\text { Sample } \\
\text { Orientation }\end{array}$ \\
\hline 7 & Core & N/A & N/A & Vertical \\
\hline
\end{tabular}

Avg. $\mathrm{k}$ at $20^{\circ} \mathrm{C} \quad 3.6 \mathrm{E}-08 \mathrm{~cm} / \mathrm{se}$

$\mathrm{a}_{\mathrm{a}}=0.76712 \mathrm{~cm}^{2}$

$\mathrm{A}=\quad 45.28 \mathrm{~cm}^{2}$

$\mathrm{L}=\quad 11.32 \mathrm{~cm}$

$\mathrm{S}=\mathrm{L} / \mathrm{A}=0.250021 / \mathrm{cm}$ $\mathrm{a}_{\mathrm{p}}=0.031416 \mathrm{~cm}^{2}$

$\mathrm{M}_{1}=0.03018$

$\mathrm{M}_{2}=\quad 1.04095$

$\mathrm{C}=\mathrm{M}_{1} \mathrm{~S} /\left(\mathrm{G}_{\mathrm{Hg}}-1\right)=0.0006003$ for $15^{\circ}$ to $25^{\circ}$
Remarks:

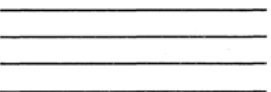

RCN: SRS256 Page 40 of 46 


\section{amec $^{\theta}$}

\section{HYDRAULIC CONDUCTIVITY}

$\begin{array}{llll}\text { Project No. } & \mathbf{6 1 5 5 - 0 8 - 0 0 3 1 . 3 4} & \text { Tested By } & \text { JEL } \\ \text { Project Name } & \text { SDU-6 Task 3 Grout Samples } & \text { Test Date } & 7 / 17 / 2012 \\ \text { Boring No. } & H 0.5-2 & \text { Reviewed By } & J W G \varepsilon \text { V } \\ \text { Sample No. } & H 0.5-2 & \text { Review Date } & 8 / 1 / 2012 \\ \text { Sample Depth } & \text { N/A } & \text { Lab No. } & 11624\end{array}$

Sample Description Grout Core

ASTM D5084 - Method F (CVFH)

\begin{tabular}{|l|l|}
\hline Sample Type: & Core \\
\hline Sample Orientation: & Vertical \\
\hline Initial Water Content, \%: & 42.9 \\
\hline Wet Unit Weight, pcf: & 108.2 \\
\hline Dry Unit Weight, pcf: & 75.7 \\
\hline Compaction, \%: & N/A \\
\hline Hydraulic Conductivity, cm/sec. @20 ${ }^{\circ} \mathrm{C}$ & $4.7 E-08$ \\
\hline
\end{tabular}

Remarks: 
SRNL-STI-2012-00522

Revision 0

PERMEABILITY TEST

(ASTM D5084 - 03) (Method F, Constant Volume Falling Head)

$\operatorname{amec}^{\theta}$

Project Number 6155-08-0031.34

Tested By JEL

Project Name SDU-6 Task 3 Grout Samples Test Date 07/17/12

$\begin{array}{lll}\text { Boring No. } & \mathrm{H} 0.5-2 & \begin{array}{l}\text { Reviewed By JW ffC } \\ \text { Review Date } 08 / 01 / 12\end{array} \\ \text { Sample No. } & \mathrm{H} 0.5-2 & \end{array}$

Sample Depth N/A Lab No. 11624

Sample Description Grout Core

\begin{tabular}{|c|r|c|r|c|r|r|}
\hline \multicolumn{4}{|c|}{ Initial Sample Data } & \multicolumn{3}{c|}{ Final Sample Data } \\
\hline \multicolumn{2}{|c|}{ Length, in } & \multicolumn{2}{|c|}{ Diameter, in } & Pan No. & N/A \\
\hline Location 1 & 4.643 & Location 1 & 2.962 & Wet Soil+Pan, grams & 909.53 \\
\hline Location 2 & 4.657 & Location 2 & 2.956 & Dry Soil + Pan, grams & 636.35 \\
\hline Location3 & 4.648 & Location 3 & 2.965 & Pan Weight, grams & 0 \\
\hline Average & 4.649 & Average & 2.961 & Moisture Content, \% & 42.9 \\
\hline Volume, in & 32.02 & Wet Soil + Tare, grams & 909.35 & Dry Unit Weight, pcf & 75.7 \\
\hline SG Assumed & 2.40 & Tare Weight, grams & 0.00 & Saturation, \% & 105.4 \\
\hline Soil Sample Wt., g & 909.35 & Dry Soil +Tare, grams & 636.35 & Diameter, in. & N/A \\
\hline Dry UW, pcf & 75.7 & Moisture Content, \% & 42.9 & Length, in. & N/A \\
\hline Saturation, \% & 105.3 & & & Volume, in & N/A \\
\hline
\end{tabular}

Consolidation Chamber Pressure, psi 70 Back Pressure, psi

Confining Pressure, psi 10

Initial Burett Reading

Final Burett Reading

Final Burett Reading

Volume Change, cc

Permeant used

\begin{tabular}{l}
$\frac{10}{60}$ \\
\hline 10 \\
\hline 0 \\
\hline 0 \\
\hline 0 \\
\hline
\end{tabular}

\begin{tabular}{|r|r|r|r|r|r|r|r|r|r|}
\hline $\begin{array}{c}\text { Elapsed Time } \\
(\mathrm{sec})\end{array}$ & $\begin{array}{c}\mathrm{z}_{\mathrm{o}} \\
(\mathrm{cm})\end{array}$ & $\begin{array}{c}\mathrm{za} \\
(\mathrm{cm})\end{array}$ & $\begin{array}{c}\mathrm{zb} \\
(\mathrm{cm})\end{array}$ & $\begin{array}{c}\Delta \mathrm{z}_{\mathrm{p}} \\
(\mathrm{cm})\end{array}$ & $\begin{array}{c}\text { Temp } \\
\left({ }^{\circ} \mathrm{C}\right)\end{array}$ & $\begin{array}{c}\text { Intial } \\
\text { Hydraulic } \\
\text { Gradient }\end{array}$ & $\begin{array}{c}\text { Final } \\
\text { Hydraulic } \\
\text { Gradient }\end{array}$ & $\begin{array}{c}\mathrm{k} \\
\mathrm{cm} / \mathrm{sec}\end{array}$ & $\begin{array}{c}\mathrm{k} \\
\mathrm{cm} / \mathrm{sec} \\
\mathrm{at} \mathrm{20}{ }^{\circ} \mathrm{C}\end{array}$ \\
\hline 2100 & 1.60 & 24.20 & 20.40 & 3.80 & 21.9 & 24.1 & 19.8 & $5.85 \mathrm{E}-08$ & $5.59 \mathrm{E}-08$ \\
\hline 2610 & 1.60 & 24.20 & 19.80 & 4.40 & 21.9 & 24.1 & 19.2 & $5.54 \mathrm{E}-08$ & $5.29 \mathrm{E}-08$ \\
\hline 3240 & 1.60 & 24.20 & 19.10 & 5.10 & 21.9 & 24.1 & 18.4 & $5.27 \mathrm{E}-08$ & $5.04 \mathrm{E}-08$ \\
\hline 3900 & 1.60 & 24.20 & 18.40 & 5.80 & 22.0 & 24.1 & 17.6 & $5.09 \mathrm{E}-08$ & $4.85 \mathrm{E}-08$ \\
\hline 990 & 1.60 & 24.50 & 23.00 & 1.50 & 22.0 & 24.4 & 22.7 & $4.55 \mathrm{E}-08$ & $4.34 \mathrm{E}-08$ \\
\hline 1700 & 1.60 & 24.50 & 22.10 & 2.40 & 22.0 & 24.4 & 21.7 & $4.34 \mathrm{E}-08$ & $4.13 \mathrm{E}-08$ \\
\hline 2310 & 1.60 & 24.50 & 21.45 & 3.05 & 22.0 & 24.4 & 21.0 & $4.12 \mathrm{E}-08$ & $3.93 \mathrm{E}-08$ \\
\hline
\end{tabular}

$\begin{aligned} & \begin{array}{rr}\text { No. of Trials } & \begin{array}{c}\text { Samp } \\ \text { Typp }\end{array} \\ \hline 7 & \text { Cor } \\ \mathrm{a}_{\mathrm{a}}= & 0.76712 \mathrm{~cm}^{2} \\ \mathrm{~A}= & 44.43 \mathrm{~cm}^{2} \\ \mathrm{~L}= & 11.81 \mathrm{~cm}^{2} \\ \mathrm{~S}=\mathrm{L} / \mathrm{A}= & 0.26582 \mathrm{l} / \mathrm{cm}\end{array}\end{aligned}$

Mample Density Compaction Sample

\begin{tabular}{c|c|c|c|} 
Type & $(\mathrm{pcf})$ & $\%$ & Orientation \\
\hline
\end{tabular}

Vertical

Avg. $\mathrm{k}$ at $20^{\circ} \mathrm{C} \quad \underline{4.7 \mathrm{E}-08 \mathrm{~cm} / \mathrm{sec}}$

$=0.265821 / \mathrm{cm}$

$\mathrm{a}_{\mathrm{p}}=0.031416 \mathrm{~cm}^{2}$

$\mathrm{M}_{1}=0.03018$

$\mathrm{M}_{2}=\quad 1.04095$

$\mathrm{C}=\mathrm{M}_{1} \mathrm{~S} /\left(\mathrm{G}_{\mathrm{Hg}}-1\right)=0.0006382$ for $15^{\circ}$ to $25^{\circ}$

Remarks 


\section{amec $^{\theta}$}

\section{HYDRAULIC CONDUCTIVITY}

$\begin{array}{llll}\text { Project No. } & \text { 6155-08-0031.34 } & \text { Tested By } & \text { JEL } \\ \text { Project Name } & \text { SDU-6 Task 3 Grout Samples } & \text { Test Date } & 7 / 19 / 2012 \\ \text { Boring No. } & \text { H0.5-3 } & \text { Reviewed By } & \text { JW Øלf } \\ \text { Sample No. } & \text { H0.5-3 } & \text { Review Date } & 8 / 1 / 2012 \\ \text { Sample Depth } & \text { N/A } & \text { Lab No. } & 11625\end{array}$

Sample Description Grout Core

ASTM D5084 - Method F (CVFH)

\begin{tabular}{|l|l|}
\hline Sample Type: & Core \\
\hline Sample Orientation: & Vertical \\
\hline Initial Water Content, \%: & 42.3 \\
\hline Wet Unit Weight, pcf: & 107.5 \\
\hline Dry Unit Weight, pcf: & 75.5 \\
\hline Compaction, \%: & N/A \\
\hline Hydraulic Conductivity, cm/sec. @20 C & $3.7 E-08$ \\
\hline
\end{tabular}

Remarks: 
SRNL-STI-2012-00522

Revision 0

PERMEABILITY TEST

(ASTM D5084 - 03) (Method F, Constant Volume Falling Head)

amec $^{\theta}$

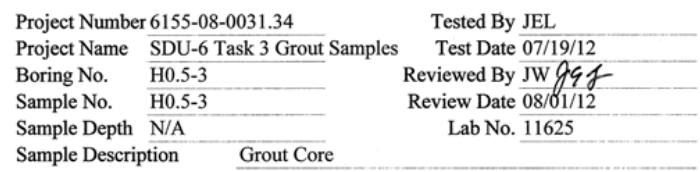

\begin{tabular}{l|c}
\hline Initial Sample Data & Final Sample Data \\
\hline
\end{tabular}

\begin{tabular}{|c|r|c|r|c|c|}
\hline \multicolumn{4}{|c|}{ Initial Sample Data } & \multicolumn{2}{c|}{ Final Sample Data } \\
\hline \multicolumn{2}{|c|}{ Length, in } & \multicolumn{2}{|c|}{ Diameter, in } & Pan No. & N?A \\
\hline Location 1 & 4.575 & Location 1 & 3.030 & Wet Soil+Pan, grams & 903.06 \\
\hline Location 2 & 4.540 & Location 2 & 2.960 & Dry Soil + Pan, grams & 634.31 \\
\hline Location3 & 4.560 & Location 3 & 2.978 & Pan Weight, grams & \\
\hline Average & 4.558 & Average & 2.989 & Moisture Content, \% & 42.4 \\
\hline Volume, in & 31.99 & Wet Soil + Tare, grams & 902.84 & Dry Unit Weight, pcf & 75.5 \\
\hline SG Assumed & 2.40 & Tare Weight, grams & 0.00 & Saturation, \% & 103.5 \\
\hline Soil Sample Wt., g & 902.84 & Dry Soil +Tare, grams & 634.31 & Diameter, in. & N/A \\
\hline Dry UW, pef & 75.5 & Moisture Content, \% & 42.3 & Length, in. & N/A \\
\hline Saturation, \% & 103.4 & & & Volume, in & N/A \\
\hline
\end{tabular}

Consolidation

Chamber Pressure, psi

Back Pressure, psi

Confining Pressure, psi 10

Initial Burett Reading

Final Burett Reading

Volume Change, cc

Permeant used

$\frac{\frac{70}{60}}{\frac{10}{0}}$

\begin{tabular}{|r|c|c|c|c|c|c|c|c|c|}
\hline $\begin{array}{c}\text { Elapsed Time } \\
(\mathrm{sec})\end{array}$ & $\begin{array}{c}\mathrm{z}_{\mathrm{o}} \\
(\mathrm{cm})\end{array}$ & $\begin{array}{c}\mathrm{za} \\
(\mathrm{cm})\end{array}$ & $\begin{array}{c}\mathrm{zb} \\
(\mathrm{cm})\end{array}$ & $\begin{array}{c}\Delta \mathrm{z}_{\mathrm{p}} \\
(\mathrm{cm})\end{array}$ & $\begin{array}{c}\text { Temp } \\
\left({ }^{\circ} \mathrm{C}\right)\end{array}$ & $\begin{array}{c}\text { Intial } \\
\text { Hydraulic } \\
\text { Gradient }\end{array}$ & $\begin{array}{c}\text { Final } \\
\text { Hydraulic } \\
\text { Gradient }\end{array}$ & $\begin{array}{c}\mathrm{k} \\
\mathrm{cm} / \mathrm{sec}\end{array}$ & $\begin{array}{c}\mathrm{k} \\
\mathrm{cm} / \mathrm{sec} \\
\mathrm{at} 20^{\circ} \mathrm{C}\end{array}$ \\
\hline 1860 & 1.50 & 22.40 & 19.85 & 2.55 & 22.4 & 22.7 & 19.8 & $4.48 \mathrm{E}-08$ & $4.23 \mathrm{E}-08$ \\
\hline 2880 & 1.50 & 22.40 & 18.80 & 3.60 & 22.4 & 22.7 & 18.6 & $4.21 \mathrm{E}-08$ & $3.98 \mathrm{E}-08$ \\
\hline 1120 & 1.50 & 23.00 & 21.50 & 1.50 & 22.3 & 23.3 & 21.6 & $4.13 \mathrm{E}-08$ & $3.91 \mathrm{E}-08$ \\
\hline 2490 & 1.50 & 23.00 & 20.20 & 2.80 & 22.4 & 23.3 & 20.2 & $3.59 \mathrm{E}-08$ & $3.39 \mathrm{E}-08$ \\
\hline 3360 & 1.50 & 23.00 & 19.30 & 3.70 & 22.4 & 23.3 & 19.2 & $3.61 \mathrm{E}-08$ & $3.41 \mathrm{E}-08$ \\
\hline 1000 & 1.50 & 23.40 & 22.10 & 1.30 & 22.5 & 23.8 & 22.3 & $3.92 \mathrm{E}-08$ & $3.69 \mathrm{E}-08$ \\
\hline 2100 & 1.50 & 23.40 & 21.00 & 2.40 & 22.4 & 23.8 & 21.1 & $3.54 \mathrm{E}-08$ & $3.34 \mathrm{E}-08$ \\
\hline
\end{tabular}

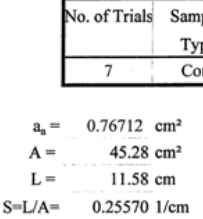

Avg. $\mathrm{k}$ at $20^{\circ} \mathrm{C} \quad \underline{3.7 \mathrm{E}-08 \mathrm{~cm} / \mathrm{sec}}$

Remarks

$\mathrm{a}_{\mathrm{p}}=0.031416 \mathrm{~cm}^{2}$

$\mathrm{M}_{1}=0.03018$

$\mathrm{M}_{2}=1.04095$

$\mathrm{S}=\mathrm{L} / \mathrm{A}=\quad 0.255701 / \mathrm{cm}$

$\mathrm{C}=\mathrm{M}_{1} \mathrm{~S} /\left(\mathrm{G}_{\mathrm{Hg}^{-}}-1\right)=0.0006139$ for $15^{\circ}$ to $25^{\circ}$

RCN: SRS256 Page 44 of 46 
SRNL-STI-2012-00522

Revision 0

SDU-6 Task 3 Grout Samples AC54317N DO34 AMEC Project No. 6155-08-0031

August 1, 2012

ATTACHMENT 2

Page 3 of 3

RCN: SRS256

Page 45 of 46 
SRNL-STI-2012-00522

Revision 0

Equipment List

SRNS Delivery Order No. 34

Subcontract No. AC54317N

\begin{tabular}{|c|c|}
\hline Equipment Name & Laboratory ID \\
\hline Oven & 109 \\
\hline Balance & 416 \\
\hline Thermometer & 2866 \\
\hline Caliper & 2373 \\
\hline Pressure Transducers & 3638 \\
\hline & \\
\hline & \\
\hline & \\
\hline & \\
\hline & \\
\hline & \\
\hline
\end{tabular}

RCN: SRS256 


\section{Distribution:}

P. M. Almond, 773-43A

J. M. Bricker, 704-27S

T. E. Brooks, 707-14E

A. D. Cozzi, 999-W

W. E. Daniel, 999-W

R. E. Eibling, 999-W

T. L. Fellinger, 704-26S

S. D. Fink, 773-A

K. M. Fox, 999-W

B. J. Giddings, 786-5A

E. K. Hansen, 999-W

C. C. Herman, 999-W

T. H. Huff, 707-13E

P. R. Jackson, DOE-SR, 703-46A

C. A. Langton, 773-43A

J. N. Leita, 704-Z

S. L. Marra, 773-A

D. H. Miller, 999-W

J. E. Occhipinti, 704-S

F. M. Pennebaker, 773-42A

B. R. Pickenheim, 999-W

M. M. Reigel, 999-W

K. H. Rosenberger, 705-1C

M. G. Serrato, 773-42A

F. M. Smith, 705-1C

A. V. Staub, 704-27S

D. B. Stefanko, 773-43A

K. H. Subramanian, 766-H

J. R. Tihey, 704-Z

Records Administration (EDWS) 